Prepared in cooperation with the California Department of Water Resources

Construction and Analysis of a Giant Gartersnake (Thamnophis gigas) Population Projection Model

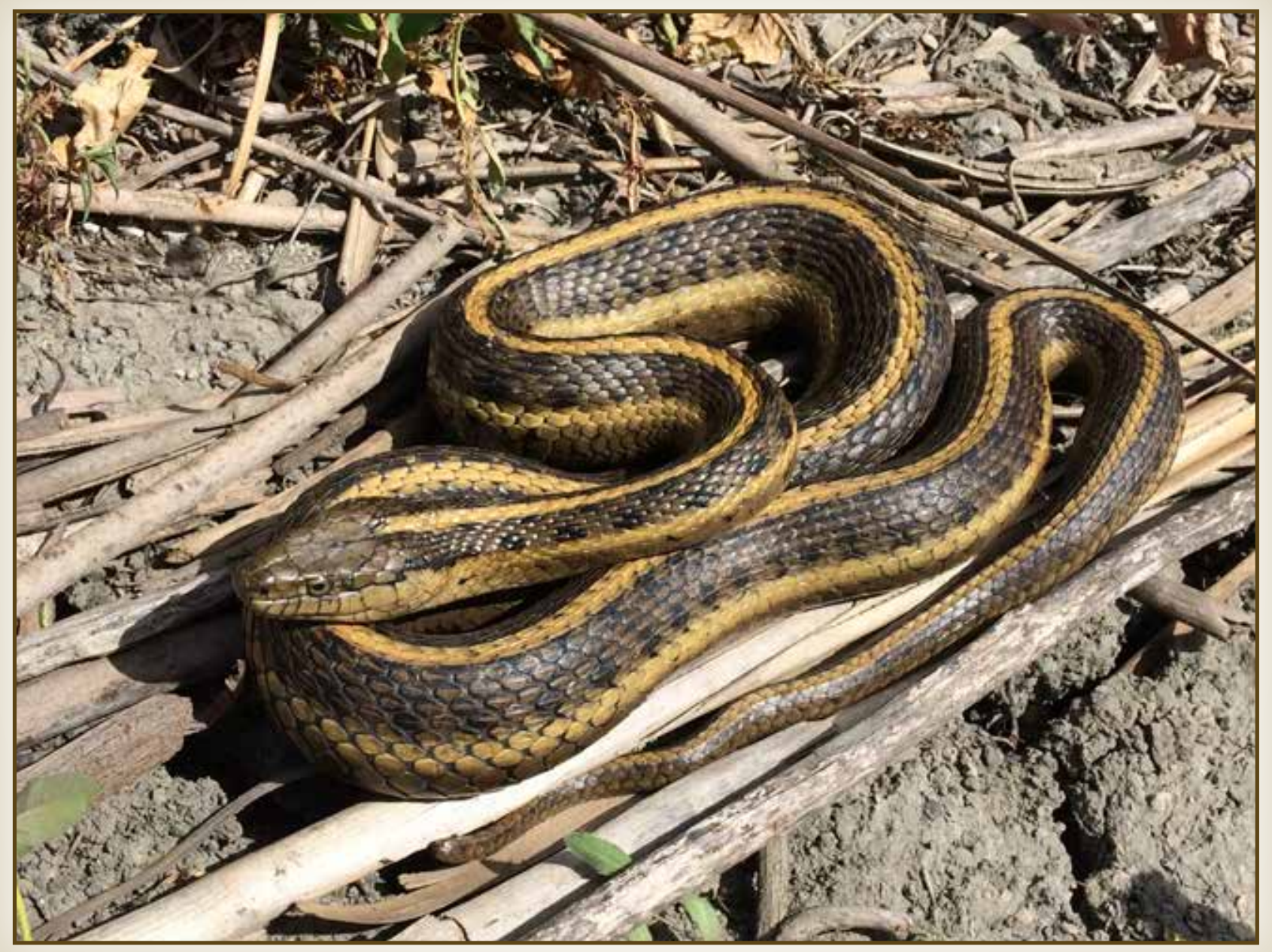

Open-File Report 2017-1164 
Cover: Photograph showing an adult female giant gartersnake (Thamnophis gigas), Sacramento Valley, California, representing the importance of large adult female snakes for population persistence.

Photograph by Alexandria M. Fulton, U.S. Geological Survey, May 10, 2017. 


\section{Construction and Analysis of a Giant Gartersnake (Thamnophis gigas) Population Projection Model}

By Jonathan P. Rose, Julia S.M. Ersan, Glenn D. Wylie, Michael L. Casazza, and Brian J. Halstead

Prepared in cooperation with the California Department of Water Resources

Open-File Report 2017-1164

U.S. Department of the Interior

U.S. Geological Survey 


\section{U.S. Department of the Interior \\ RYAN K. ZINKE, Secretary}

\section{U.S. Geological Survey \\ William H. Werkheiser, Deputy Director \\ exercising the authority of the Director}

U.S. Geological Survey, Reston, Virginia: 2018

For more information on the USGS—-the Federal source for science about the Earth, its natural and living resources, natural hazards, and the environment-visit https://www.usgs.gov/ or call 1-888-ASK-USGS (1-888-275-8747).

For an overview of USGS information products, including maps, imagery, and publications, visit https:/store.usgs.gov.

Any use of trade, firm, or product names is for descriptive purposes only and does not imply endorsement by the U.S. Government.

Although this information product, for the most part, is in the public domain, it also may contain copyrighted materials as noted in the text. Permission to reproduce copyrighted items must be secured from the copyright owner.

Suggested citation:

Rose, J.P., Ersan, J.S.M., Wylie, G.D., Casazza, M.L., and Halstead, B.J., 2018, Construction and analysis of a giant gartersnake (Thamnophis gigas) population projection model: U.S. Geological Survey Open-File Report 2017-1164, 98 p., https://doi.org/10.3133/ofr20171164.

ISSN 2331-1258 (online) 


\section{Acknowledgments}

We thank the California Department of Water Resources for supporting this project. We also thank the U.S. Fish and Wildlife Service and the Natomas Basin Conservancy for funding projects whose data were used in this report. We thank the Natomas Basin Conservancy, Colusa National Wildlife Refuge, Wildlands, Inc., and several cooperative landowners for access to the study sites. We also thank the giant gartersnake Technical Review Committee for providing feedback and guidance for this study, Julie Yee and Brian Prochazka for reviewing this report, and Tim Tinker and Elizabeth Gallegos for reviews of sections 1 and 2, respectively. We are grateful to Eric Hansen, Rick Scherer and Maxwell Joseph for sharing data and model results on giant gartersnake reproduction. Dr. Ray Wack of the University of California, Davis and the Sacramento Zoo conducted the X-rays and interpreted the number of embryos in each radiograph for the fecundity analysis. We thank Lisa Parker for administrative support.

This study would not have been possible without the work of numerous biological technicians who collected field data on giant gartersnakes for the U.S. Geological Survey for many years. This work was performed under Institutional Animal Care and Use Committee protocol WERC-2014-01 and as stipulated in California Department of Fish and Wildlife Scientific Collecting Permit 10779 and U.S. Fish and Wildlife Service Recovery Permits TE020548-5 and TE-157216-2. 
This page left intentionally blank 


\section{Contents}

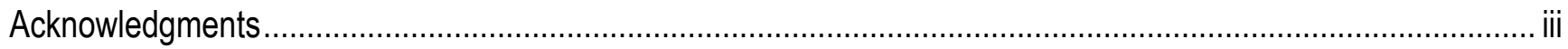

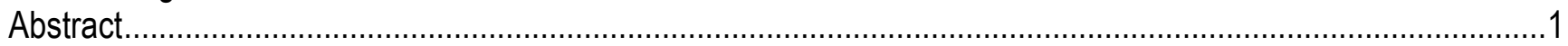

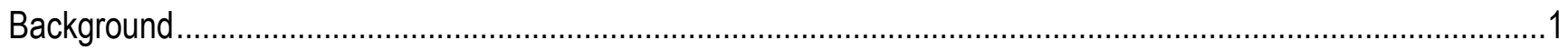

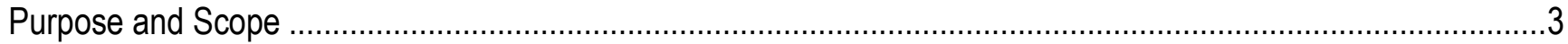

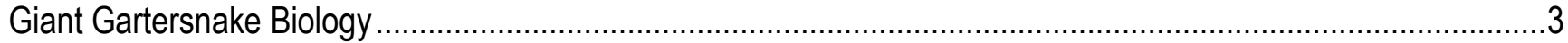

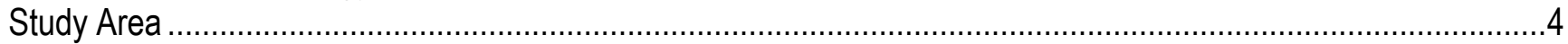

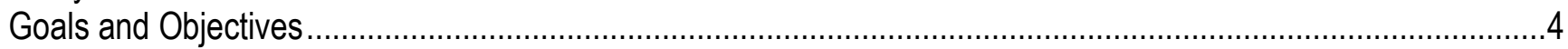

Section 1: Fitting a von Bertalanffy Growth Model for Giant Gartersnakes ....................................................5

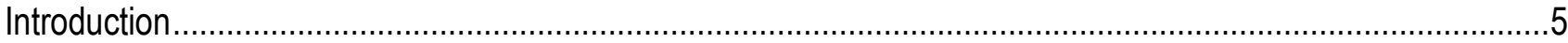

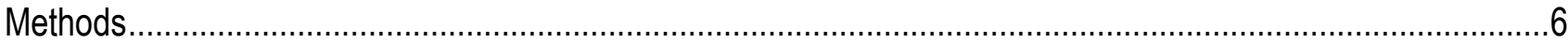

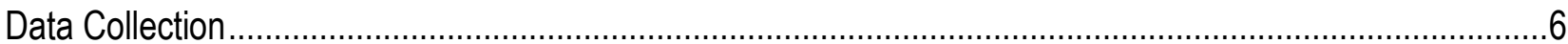

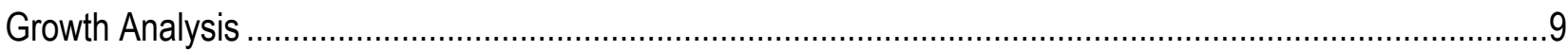

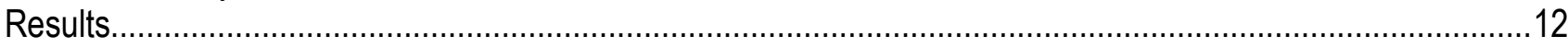

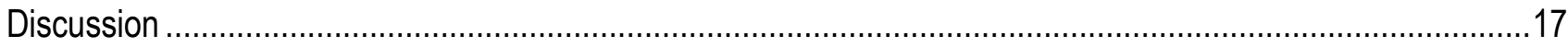

Section 2: Reproductive Frequency and Size-Dependence of Fecundity in the Giant Gartersnake....................19

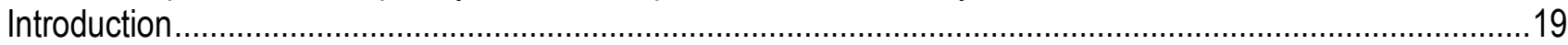

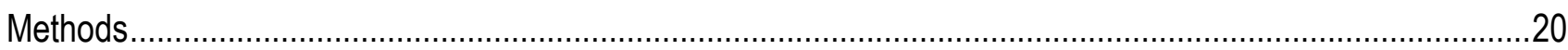

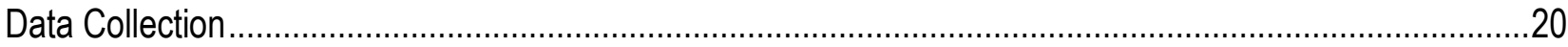

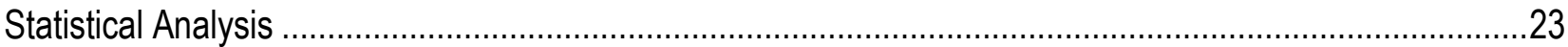

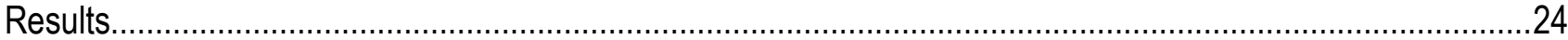

Litter Size, Neonate Size, and Maternal Size ..................................................................................25

Annual Proportion of Gravid Females and Reproductive Frequency......................................................29

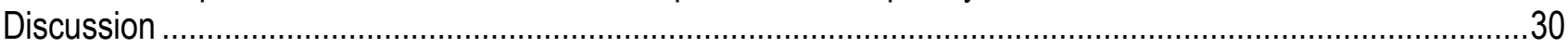

Section 3: Integrating Growth and Capture-Mark-Recapture Models to Estimate Size-Dependent

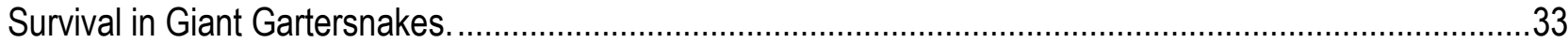

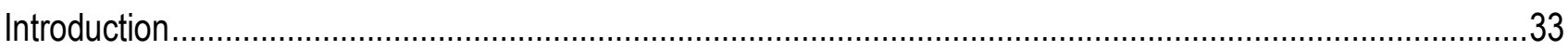

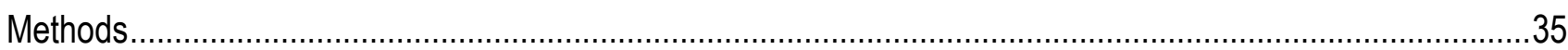

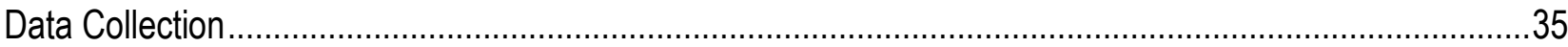

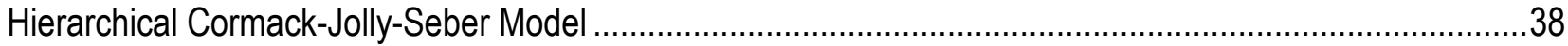

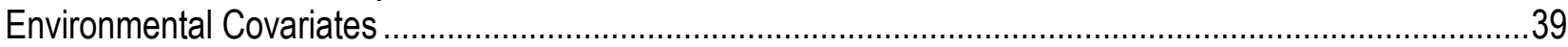

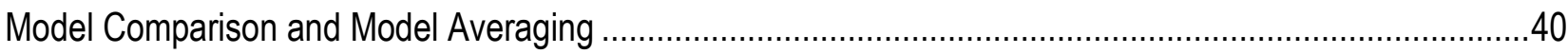

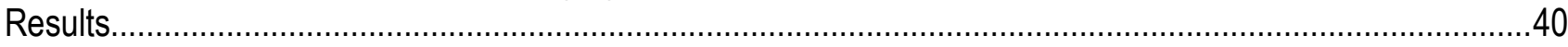

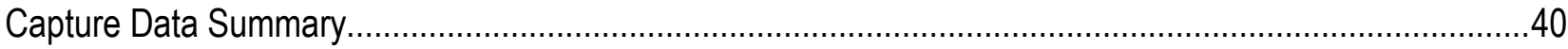

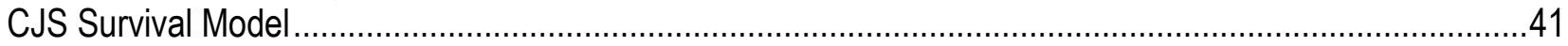

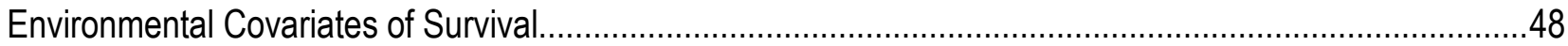

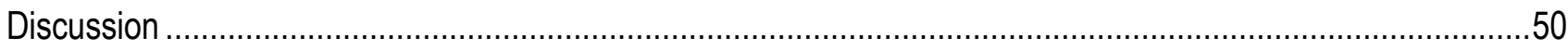

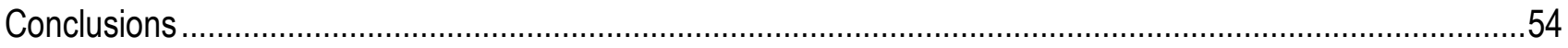


Section 4: Development and Elasticity Analysis of an Integral Projection Model for the

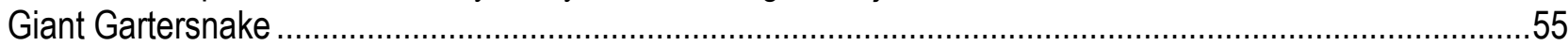

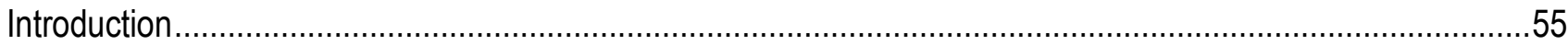

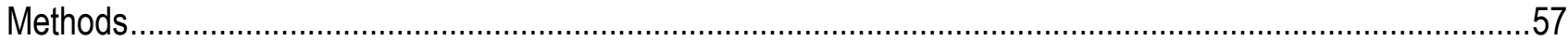

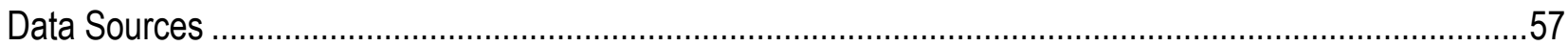

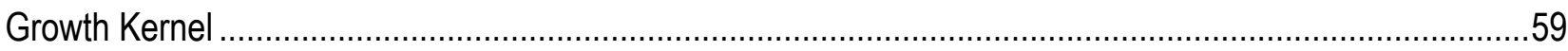

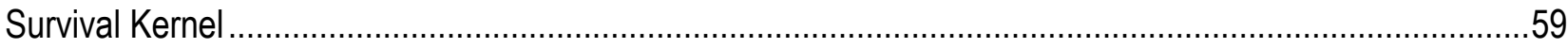

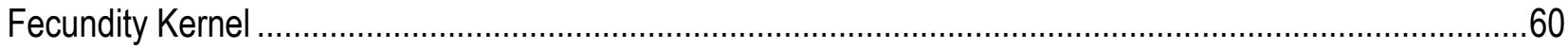

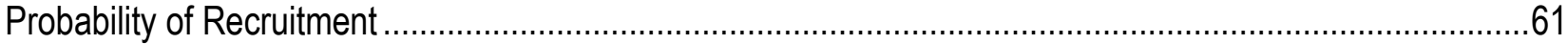

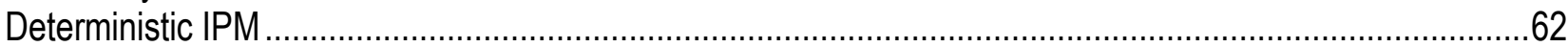

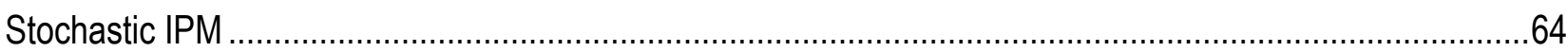

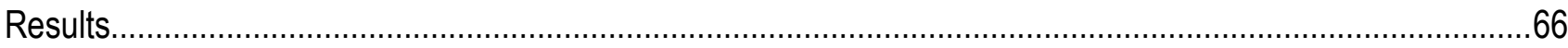

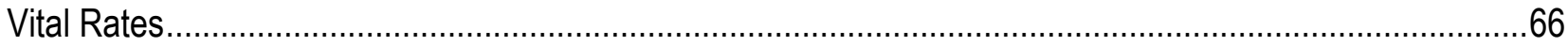

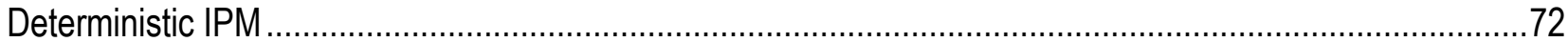

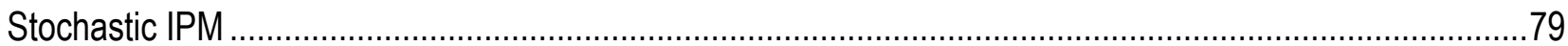

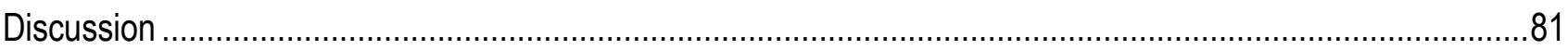

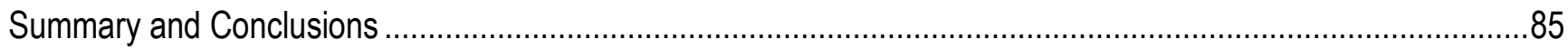

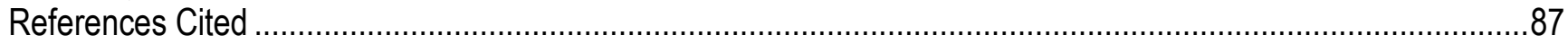

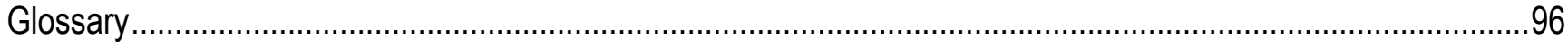

\section{Figures}

Figure 1. Location of study sites in the Sacramento Valley of California.

Figure 2. Growth curves for an average female (solid line) and male (dashed line) giant gartersnake in the

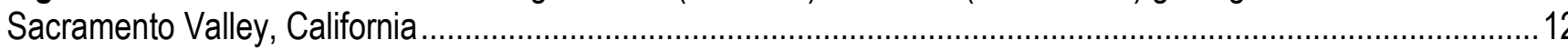

Figure 3. Effect of site on the von Bertalanffy growth coefficient, $k$, for giant gartersnakes at eight sites in the Sacramento Valley, California.

Figure 4. Effect of year on the von Bertalanffy growth coefficient, $k$, for giant gartersnakes in the Sacramento Valley, California, 1995-2015.

Figure 5. Effect of site on asymptotic length of giant gartersnakes in the Sacramento Valley, California.............14

Figure 6. Interaction between fish and frog abundance and the growth coefficient, $k \ldots \ldots \ldots \ldots \ldots \ldots \ldots \ldots \ldots \ldots \ldots \ldots \ldots . . . . .16$

Figure 7. Location of study sites for giant gartersnake fecundity in the Sacramento Valley of California.............22

Figure 8. Maternal snout-vent length of the giant gartersnake in relation to litter size for data from the current study in Sacramento Valley, California; Halstead and others (2011b); and Hansen and Hansen (1990)

Figure 9. Posterior probability of slope parameters for the effect of maternal snout-vent length (SVL) of the giant gartersnake on (a) neonate mass or (c) neonate SVL, and for the effect of total litter size on (b) neonate mass or (d) neonate SVL. $\beta_{F e c}$ represents the effect of litter size on neonate size, and $\beta$ svL represents the effect of maternal SVL on neonate size.....

Figure 10. Location of capture-mark-recapture study sites within the Sacramento Valley, California. (NWR, National Wildlife Refuge).

Figure 11. Model-averaged relationships between snake size and (a) daily capture probability ( $p$ ) and (b) apparent annual survival $(\phi)$.

Figure 12. Mean size-survival relationships for each site in Sacramento Valley, California, along with the average of all sites.

Figure 13. Average annual survival probability, $\phi$, for each site in Sacramento Valley, California........................45

Figure 14. Average annual survival probability, $\phi$, by site in Sacramento Valley, California, 
Figure 15. Average annual survival probability, $\phi$, by site in Sacramento Valley, California,

2011-15.

Figure 16. Model-averaged relationship between snake survival and (a) fish abundance an

(b) percent cover of emergent vegetation, Sacramento Valley, California

Figure 17. Growth trajectory and corresponding growth kernel for giant gartersnakes, Sacramento Valley,

California.

Figure 18. Size-survival relationship and survival kernel for giant gartersnakes, Sacramento Valley,

California.

Figure 19. The fecundity kernel and its component models for giant gartersnake, Sacramento Valley,

California.

Figure 20. Estimated probability of recruitment for neonate giant gartersnakes from 2011 to 2015 at four sites in the Natomas Basin: (a) Natomas 1, (b) Natomas 2, (c) Natomas 3, (d) Natomas 5, Sacramento Valley, California.

Figure 21. Deterministic Integral Projection Model kernels $\left(p_{\text {rec }}=0.3\right)$ for giant gartersnakes, Sacramento Valley, California...

Figure 22. Deterministic Integral Projection Model diagnostics $\left(p_{\text {rec }}=0.3\right)$ for giant gartersnake,

Sacramento Valley, California.....

Figure 23. Elasticity surfaces for the U.S. Geological Survey deterministic Integral Projection Model based primarily on U.S. Geological Survey project data for giant gartersnakes, Sacramento Valley, California

Figure 24. Elasticity surfaces for the "Hansen" deterministic Integral Projection Model based

primarily on survival and reproductive data from Hansen and others (2017).

Figure 25. Elasticity surface for the stochastic U.S. Geological Survey Integral Projection Model .80

\section{Tables}

Table 1. Duration of sampling and number of growth increments of giant gartersnakes measured by site, Sacramento Valley, California.

Table 2. Prior distributions for growth model parameters for giant gartersnakes in Sacramento Valley, California.....

Table 3. Sampling period, number of individuals, and sources of litter size estimates for the giant gartersnakes in Sacramento Valley, California

Table 4. Characteristics of examined female giant gartersnakes and their litters, Sacramento Valley, California.

Table 5. Parameter estimates from a Poisson regression model of the effect of maternal snout-vent length on litter size of the giant gartersnake, Sacramento Valley, California..

Table 6. Parameter estimates from a regression model of the effects of giant gartersnake maternal snout-vent length and litter size on neonate mass and snout-vent length, Sacramento Valley, California

Table 7. Number of giant gartersnakes examined by X-ray and number gravid, by month, Sacramento Valley, California, 2014-2016....

Table 8. Summary of years and number of individual giant gartersnakes sampled at each site in

Sacramento Valley, California, used in capture-mark-recapture analysis .

Table 9. Cormack-Jolly-Seber model parameters, descriptions, and prior distributions

Table 10. The top eight models with posterior probability greater than or equal to 0.05 for the capture probability and survival of giant gartersnakes from 10 sites in the Sacramento Valley, California, sampled from 1999 to 2016

Table 11. Model-averaged parameter estimates and 95-percent credible intervals from the Cormack-Jolly-Seber model with all capture-mark-recapture data on giant gartersnakes collected from 10 sites in Sacramento Valley, California, from 1999 to 2016. 
Table 12. The top eight models with posterior probability greater than or equal to 0.007 for the effect of environmental covariates on survival of giant gartersnakes from eight sites in the Sacramento Valley, California, sampled from 2011 to 2016

Table 13. Model-averaged parameter estimates and 95-percent credible intervals for environmental covariates on survival from the Cormack-Jolly-Seber model using capture-mark-recapture data on giant gartersnakes collected in Sacramento Valley, California, 2011-2016.

Table 14. Vital rate models, parameters, and their primary and secondary sources

Table 15. Demographic model parameters and estimates of their mean and standard deviation for giant gartersnakes in Sacramento Valley, California

Table 16. Mean and annual standard deviation of time-varying parameters used in the stochastic Integral

Projection Model

Table 17. Elasticity of the population growth rate, $\lambda$, of giant gartersnakes in Sacramento Valley, California, to perturbation of vital rate model parameters for the deterministic Integral Projection Model, based primarily on U.S. Geological Survey data, for four values of the probability of recruitment, $p_{\text {rec }}$......

Table 18. Elasticity of the population growth rate, $\lambda$, of giant gartersnakes in Sacramento Valley, California, to perturbation of vital rate model parameters for the deterministic Integral Projection Model, based primarily on reproductive and survival data from Hansen and others $(2015,2017)$, for four values of $p_{\text {rec. }}$

\section{Conversion Factors}

International System of Units to U.S. customary units

\begin{tabular}{|c|c|c|}
\hline Multiply & By & To obtain \\
\hline \multicolumn{3}{|c|}{ Length } \\
\hline millimeter (mm) & 0.0393701 & inch (in.) \\
\hline meter $(\mathrm{m})$ & 3.28084 & foot $(\mathrm{ft})$ \\
\hline kilometer (km) & 0.621371 & mile (mi) \\
\hline \multicolumn{3}{|c|}{ Mass } \\
\hline $\operatorname{gram}(\mathrm{g})$ & 0.00220462 & ounce, avoirdupois (oz) \\
\hline kilogram (kg) & 2.20462 & pound avoirdupois (lb) \\
\hline
\end{tabular}

\section{Abbreviations}

$\begin{array}{ll}\text { CMR } & \text { capture-mark-recapture } \\ \text { CRI } & \text { credible interval } \\ \text { IPM } & \text { Integral Projection Model } \\ \text { JAGS } & \text { Just Another Gibbs Sampler } \\ \text { MCMC } & \text { Markov Chain Monte Carlo } \\ \text { NWR } & \text { National Wildlife Refuge } \\ \text { PIT } & \text { passive integrated transponder } \\ \text { PRISM } & \text { Parameter-elevation Relationships on Independent Slopes Model } \\ \text { PVA } & \text { population viability analysis } \\ \text { SVL } & \text { snout-vent length }\end{array}$




\title{
Construction and Analysis of a Giant Gartersnake (Thamnophis gigas) Population Projection Model
}

\author{
By Jonathan P. Rose, Julia S.M. Ersan, Glenn D. Wylie, Michael L. Casazza, and Brian J. Halstead
}

\begin{abstract}
The giant gartersnake (Thamnophis gigas) is a state and federally threatened species precinctive to California. The range of the giant gartersnake has contracted in the last century because its wetland habitat has been drained for agriculture and development. As a result of this habitat alteration, giant gartersnakes now largely persist in and near rice agriculture in the Sacramento Valley, because the system of canals that conveys water for rice growing approximates historical wetland habitat. Many aspects of the demography of giant gartersnakes are unknown, including how individuals grow throughout their life, how size influences reproduction, and how survival varies over time and among populations. We studied giant gartersnakes throughout the Sacramento Valley of California from 1995 to 2016 using capture-mark-recapture to study the growth, reproduction, and survival of this threatened species. We then use these data to construct an Integral Projection Model, and analyze this demographic model to understand which vital rates contribute most to the growth rate of giant gartersnake populations. We find that giant gartersnakes exhibit indeterminate growth; growth slows as individuals' age. Fecundity, probability of reproduction, and survival all increase with size, although survival may decline for the largest female giant gartersnakes. The population growth rate of giant gartersnakes is most influenced by the survival and growth of large adult females, and the size at which 1 year old recruits enter the population. Our results indicate that management actions benefitting these influential demographic parameters will have the greatest positive effect on giant gartersnake population growth rates, and therefore population persistence. This study informs the conservation and management of giant gartersnakes and their habitat, and illustrates the effectiveness of hierarchical Bayesian models for the study of rare and elusive species.
\end{abstract}

\section{Background}

The limited resources available for conservation and management, and science to inform these processes, necessitates that scientists and managers identify the research questions and conservation actions that will have the greatest effect on the persistence of populations of threatened and endangered species. One tool that is commonly used to evaluate conservation actions is a population projection model. Population projection models make projections about the future state of populations on the basis of the current state and a defined set of demographic vital rates. Population projection models are perhaps best used to examine how different population processes affect future population states (Mills, 2007). 
Sensitivity analysis is a systematic way of evaluating the importance of different demographic or vital rates for the growth of the population as a whole. Sensitivity is therefore the change in population growth rate caused by a given change in a vital rate, such as survival or fecundity. Because survival is measured on a probability scale (0-1) and the number of offspring produced is measured as a number of individuals (usually the number of female offspring produced per female and typically on a much larger scale), sensitivity is often scaled to be represented as a proportional change in a demographic rate. This proportional sensitivity is called elasticity, and elasticity is used as the measure of the importance of a vital rate throughout this report. Elasticity can be used to identify critical life-history stages at which intervention to affect population growth will be most likely to succeed (Williams and others, 2002; Mills, 2007), and it is for this purpose that we employ a population projection model to study Thamnophis gigas (giant gartersnake) population biology.

Population projection models can take several forms. The original form of population projection model is the Leslie matrix model (Leslie, 1945; Caswell, 2001). A Leslie matrix is set up as a matrix of transition probabilities between ages, with survival from one age to the next on the first sub-diagonal and age-specific fecundities in the first row. Leslie matrices are appropriate for organisms that can be reliably aged and for which age-specific survival and fecundity data are available. Leslie matrix models also can be generalized to accommodate organisms for which demographic rates vary by stage or size class, rather than age (Lefkovitch, 1965; Caswell, 2001). Sizeclass or stage-based models are useful for organisms for which demographic rates depend more upon life stage than age (for example, animals that cannot reproduce until reaching a minimum size, regardless of age, or organisms [for example, fungi, some plants, many amphibians, many insects] with complex life histories). Despite their widespread use by ecologists and conservationists, there are issues with the construction and analysis of size-class or stage based models. Specifically, the arbitrary classification of continuous characteristics, such as body size, into a few discrete classes affects the conclusions of elasticity analysis for size-class based models (Enright and others, 1995; Benton and Grant, 1999).

A more recent generalization of discrete matrix population models is the Integral Projection Model (IPM; Easterling and others, 2000; Coulson, 2012; Ellner and others, 2016). IPMs allow demographic rates to vary with a continuous state variable, often individual size, rather than being tied to a discrete age or stage. IPMs are easy to parameterize using fitted regression models for growth, fecundity, and survival (Easterling and others, 2000). IPMs also can easily incorporate complex, nonlinear relationships between the state variable and the vital rates of interest (Dahlgren and others, 2011). IPMs can perform better than matrix population models for small demographic datasets (Ramula and others, 2009) and can easily be extended to accommodate demographic and environmental stochasticity and uncertainty (Rees and Ellner, 2009; Elderd and Miller, 2016). The flexibility and many appealing features of IPMs have led to their widespread use for the study of invasive and endangered species (Jongejans and others, 2011; González and others, 2013). The relation of vital rates to size, rather than age or stage, makes integral projection models appropriate for organisms that have indeterminate growth or reproduce before they reach asymptotic size $\left(\mathrm{L}_{\infty}\right.$; Wallace and others, 2013), which includes many snakes (for example, Brown and Weatherhead, 1999a, 1999b; Miller and others, 2011). Thus, IPMs are now the most appealing population projection model to use for studying the demographics of threatened and endangered snake species. 
The U.S. Geological Survey, in cooperation with the California Department of Water Resources, conducted a study of the demography of giant gartersnakes. This study aims to direct future giant gartersnake research and conservation by evaluating which demographic processes have the greatest effect on giant gartersnake population growth rates and which demographic processes are not well described by existing data or models. We accomplish this by using existing data to estimate relationships between giant gartersnake size and four key vital rates: survival, fecundity, probability of reproduction, and growth. These size vital rate relationships were used to construct an IPM for the giant gartersnake. Finally, we used elasticity analysis to explore the relative importance of different demographic vital rates for population growth.

Words presented in bold font are defined in the Glossary section of this report.

\section{Purpose and Scope}

This report summarizes the methods and findings of a study conducted by the U.S. Geological Survey, in cooperation with the California Department of Water Resources, to investigate the demography of giant gartersnakes in the Sacramento Valley of California from 1995 to 2016. The report presents vital rate models of growth, fecundity, and survival of giant gartersnakes, as well as an IPM that integrates these component models into a demographic population model.

\section{Giant Gartersnake Biology}

Giant gartersnakes are a large aquatic gartersnake species precinctive to wetlands in California's Central Valley. Giant gartersnakes are semi-aquatic and historically were present in marshes, sloughs, and other habitats with slow-moving, relatively warm water and emergent vegetation, especially Schoenoplectus acutus (tules). Giant gartersnakes currently persist in isolated remnant wetlands and rice agriculture in the Sacramento Valley (northern Central Valley; Halstead and others, 2010). Canals associated with rice agriculture can provide marsh-like habitat conditions throughout the giant gartersnake active season (late March-early October; Wylie and others, 2009), and rice fields themselves are emergent wetlands for a portion of the giant gartersnake active season. Giant gartersnakes also spend a large part of their time in terrestrial habitats during their active and inactive seasons, usually seeking cover under vegetation and vegetative litter, or underground when in terrestrial habitat (Halstead and others, 2015, 2016).

Much less is known about the demography of giant gartersnakes than other, more widespread and widely studied species in the genus Thamnophis, in part because of the difficulty in capturing this rare, elusive species (Halstead and others, 2013). The giant gartersnake is classified as a threatened species under the State (California Department of Fish and Game Commission, 1971) and Federal (U.S. Fish and Wildlife Service, 1993) Endangered Species Acts, largely as a result of the loss of wetland habitats within its range. Since its listing, a large amount of data has been collected on the biology of the giant gartersnake, including space use (Halstead and others, 2015), habitat selection (Halstead and others, 2015), thermoregulation (Wylie and others, 2009), abundance and sexual size dimorphism (Wylie and others, 2010), growth (Coates and others, 2009), reproduction (Halstead and others, 2011b), and survival (Halstead and others, 2012, Hansen and others, 2015). With the amount of demographic data available, it is now possible to construct a population projection model for the giant gartersnake and use this model to aid in the conservation of this threatened species. 


\section{Study Area}

The data used in this report come primarily from study sites distributed throughout the Sacramento Valley of California. Most of the data come from three regions, the Natomas Basin in Sacramento County and Sutter County, Gilsizer Slough in Sutter County, and the Colusa National Wildlife Refuge in Colusa County (figs. 1, 7, 10). Other USGS studies in Butte, Colusa, Glenn, Sacramento, Sutter, and Yolo Counties also contributed data on the growth and reproduction of giant gartersnakes (figs. 1, 7, 10). Additional data on giant gartersnake reproduction came from sites in the San Joaquin Valley (Hansen and Hansen, 1990; Hansen and others, 2017). More information and maps of the study sites used for the growth, fecundity, and survival components of this study are described in sections $1-3$ of this report.

\section{Goals and Objectives}

The goal of this study is to develop and analyze a population projection model, specifically an IPM, for giant gartersnakes and use it to gain insight into the relative effects of individual growth, fecundity, and survival on the growth rate of giant gartersnake populations. This goal is supported by four objectives.

1. Data from the scientific literature and field studies of giant gartersnakes and hierarchical regression models are used to estimate how growth, survival, and fecundity vary with individual body size in this species. The estimation of size dependence in each of these vital rates is described in their own section: growth (section 1), fecundity (section 2), and survival (section 3).

2. Size-dependent vital rate models for giant gartersnakes are integrated into an Integral Projection Model (IPM; section 4), which describes all possible demographic transitions and their associated uncertainty.

3. An IPM is then used to estimate the elasticity of the population growth rate to changes in the vital rates of giant gartersnakes. This elasticity analysis points to those vital rates and life stages that have the greatest effect on the growth rate of the population as a whole.

4. Finally, the results of the elasticity analysis are used to develop potential directions for future research on the most important vital rates for giant gartersnake population growth. 


\section{Section 1: Fitting a von Bertalanffy Growth Model for Giant Gartersnakes}

\section{Introduction}

Size is a fundamental trait that affects many aspects of an animal's ecology. For reptiles, body size may affect survival (Brown and Weatherhead, 1999b), fecundity (Bronikowski and Arnold, 1999), and prey availability (Shine, 1991). Many ectotherms exhibit indeterminate growth: individuals continue growing at a slower rate after reaching sexual maturity (Sebens, 1987). The adult body size of species with indeterminate growth is a direct consequence of how somatic growth rates change throughout the organism's life. The somatic growth rate and body size of a species can lend insight into its life-history strategy. In squamate reptiles, fast growth and early maturity is associated with low adult survival, whereas slow growth and delayed maturity is found in species with high adult survival rates (Shine and Charnov, 1992).

Reptiles exhibit intra-specific variability in somatic growth rates. Growth rates vary temporally among seasons (Chabreck and Joanen, 1979; Coates and others, 2009) and among years (Brown and Weatherhead, 1999a; Madsen and Shine, 2000). The growth of individuals may depend on environmental temperatures (Autumn and De Nardo, 1995) and prey availability (Madsen and Shine, 1993, 2000). Within a species, populations can exhibit variation in growth rates owing to locally adapted life-history strategies (Bronikowski and Arnold, 1999). Many reptiles exhibit differences in growth between the sexes (Dunham, 1978; Brown and Weatherhead, 1999a) and individual variability in growth rates and asymptotic size within a population (Madsen, 1988; Eaton and Link, 2011; King and others, 2016). Costs and logistical constraints limit many studies of growth in reptiles to a small area (Brown and Weatherhead, 1999a), a short time period (Tuttle and Gregory, 2012), or both. The many sources of variability in growth in reptiles highlight the risk in drawing conclusions about the growth rate of a species on the basis of short-term studies of a single population. Rather, adequately characterizing the growth of a reptile species and identifying the factors that affect growth require a long-term, multi-population study.

Given the plasticity in growth seen in many reptiles, data on somatic growth rates and body size may be invaluable for species of conservation concern. Multi-year studies of growth can reveal density dependence in a population (Lindell and Forsman, 1996; Bjorndal and others, 2000). Differences in somatic growth rates among populations can provide a more direct measure of habitat quality than abundance (Todd and Rothermel, 2006). Growth functions are an important component of size-based demographic models (Easterling and others, 2000), which are used to inform management decisions for threatened reptiles (Doak and others, 1994). Increasingly, ecologists use stochastic demographic models to capture the dynamics of populations inhabiting variable environments (Rees and Ellner, 2009). Because growth rates may vary over time and space, demographic models that are based on mean vital rates alone could lead to misguided management decisions. 
In this study, we modeled the growth of giant gartersnakes and quantified individual, spatial, and temporal variation in growth parameters. This semi-aquatic snake species is listed as threatened under California and U.S. Endangered Species Acts (California Department of Fish and Game Commission, 1971; U.S. Fish and Wildlife Service, 1993) and is endemic to the Central Valley of California, USA (Rossman and others, 1996). The Central Valley has lost greater than 90 percent of its historical wetland habitat (Huber and others, 2010), and giant gartersnakes now primarily inhabit canals associated with rice agriculture (Halstead and others, 2010), which might provide sub-optimal habitat (Wylie and others, 2010). We used data from eight sites in the Sacramento Valley (the northern portion of the Central Valley; fig. 1) over a period of 22 years to estimate somatic growth curves for giant gartersnakes. We also investigated some of the causes of observed differences in growth among individuals, sites, and years by testing for effects of sex, precipitation, temperature, and prey abundance on the growth of these snakes. We demonstrate the usefulness of hierarchical models for modeling multiple sources of variability in growth and the manner in which Bayesian analysis can be used to estimate unobserved parameters, such as measurement error. Our study provides valuable information for the conservation and management of this threatened species, contributes to our understanding of life-history strategies of Thamnophis, and highlights the importance of including variability in growth when modeling reptile populations.

\section{Methods}

\section{Data Collection}

We captured giant gartersnakes at 8 sites in the Sacramento Valley during 22 years from 1995 to 2016, with individual sites sampled during 2-15 years (fig. 1; table 1). Sites varied by the degree of human disturbance from largely undisturbed natural wetlands (Badger Creek) and wetlands managed for wildlife (Colusa National Wildlife Refuge [NWR] and Sacramento NWR) to restored wetlands and drainage canals adjacent to rice agriculture (Colusa Drain, Gilsizer Slough, Natomas Basin, Butte Farm 1, Butte Farm 2). We captured snakes by hand or by using modified aquatic funnel traps (Casazza and others, 2000; Halstead and others, 2013) placed in wetlands and canals; trap lines usually consisted of 50-100 traps. We sampled sites throughout the active season of giant gartersnakes from April through September (Wylie and others, 2009). We double marked each snake with a unique brand on its ventral scales (Winne and others, 2006) and a passive integrated transponder (PIT) tag inserted beneath the skin or into the body cavity. Snout-vent length (SVL) and sex of each snake were recorded. 


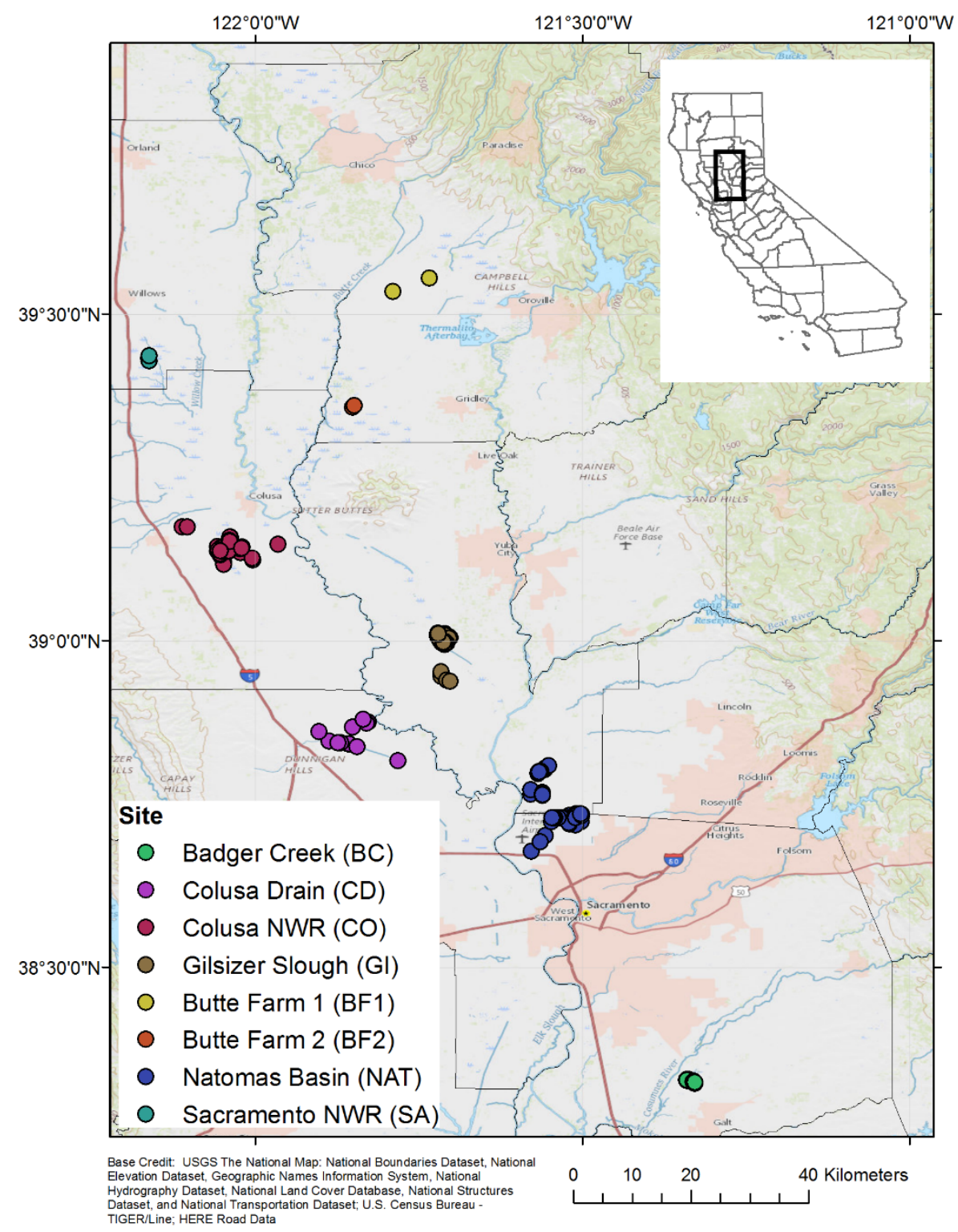

Figure 1. Location of study sites in the Sacramento Valley of California. Circles represent capture sites of snakes from which growth increment data were obtained. (Site codes are defined in table 1) 
Table 1. Duration of sampling and number of growth increments of giant gartersnakes measured by site, Sacramento Valley, California.

[NWR, National Wildlife Refuge]

\begin{tabular}{lllcc}
\hline Site & Code & Years sampled & Growth increments & Individuals \\
\hline Badger Creek & BC & $1996-1999,2002-2003$ & 43 & 38 \\
Colusa Drain & CD & $2003-2004,2014-2016$ & 17 & 17 \\
Colusa NWR & CO & $1996-2005,2012-2016$ & 239 & 181 \\
Gilsizer Slough & GI & $1995-1997,2007-2016$ & 141 & 104 \\
Butte Farm 1 & BF1 & $2009-2010,2015-2016$ & 2 & 2 \\
Butte Farm 2 & BF2 & $2015-2016$ & 8 & 8 \\
Natomas Basin & NAT & $1998-2003,2011-2016$ & 85 & 80 \\
Sacramento NWR & SA & $1997-1999$ & 3 & 3 \\
\hline
\end{tabular}

Eleven gravid females were collected from 1995 through 1997 (Halstead and others, 2011b) and 12 gravid females in 2013 and 2014 (Ersan, 2015) and kept in the lab until parturition. From these 23 litters, we measured the size of 322 neonate giant gartersnakes within 4 days of birth. We used data on size distribution at birth to infer the age at sexual maturity.

We obtained data on climatic conditions at the study sites from the Parameter-elevation Relationships on Independent Slopes Model (PRISM) climate dataset (PRISM Climate Group, 2016). The total precipitation for each year was calculated by summing monthly precipitation values over the water year, which extends from October 1 through September 30 and is a metric often used to summarize annual precipitation (for example, Diaz and Wahl, 2015). For example, the water year 2015 is composed of the total precipitation from October 1, 2014, to September 30,2015 . The number of growing degree days (above 10 degrees Celsius $\left[{ }^{\circ} \mathrm{C}\right]$ and below 37.8 ${ }^{\circ} \mathrm{C}$ ) between April 1 and September 30 of each year was calculated using the U.S. Degree-Day Mapping Calculator (Coop, 2010) and used as a measure of the thermal regime available for growth. We chose these temperature thresholds on the basis of the range of body temperatures of radio-tracked giant gartersnakes during the active season $\left(7-37^{\circ} \mathrm{C}\right)$ and the expectation that snakes are unlikely to grow at temperatures below $10^{\circ} \mathrm{C}$ (Wylie and others, 2009). Growing degree days were calculated on the basis of the daily minimum and maximum temperature using the sine wave method (Pruess, 1983).

Data on the abundance of prey within every fifth funnel trap were collected from 2003 through 2016. The fish at study sites are primarily introduced species in the families Cyprinidae, Centrarchidae, and Ictaluridae, along with Gambusia affinis (western mosquitofish) and Menidia beryllina (inland silverside), all of which are prey for giant gartersnakes (Ersan, 2015), so we summed the number of fish from all species into a "fish" covariate. Only two anuran species are present at the study sites, the native Pseudacris regilla (Pacific treefrog) and the nonnative Rana catesbeiana (=Lithobates catesbeianus; American bullfrog). Giant gartersnakes prey on both species (Ersan, 2015), so we grouped counts of larval Pacific treefrogs and American bullfrogs into a "tadpoles" covariate and counts of subadults and adults into a "frogs" covariate. 
To create an index of the abundance of prey at each site, for each year, we fit a hierarchical model with a zero-inflated Poisson response to the bycatch data. Zero-inflated Poisson models are useful when analyzing count data that do not fit a standard Poisson distribution because of an excess of zeroes (Martin and others, 2005). The number of prey captures are modeled on the basis of two parameters: the probability a trap contains prey, which is used in a binomial model, and the mean count of prey present in the trap, which is Poisson distributed. Trap lines were grouped into 19 clusters on the basis of spatial proximity, and a random effect of trap-line cluster and year within cluster for both parameters were included. The probability of prey capture was multiplied by the mean count of prey captured to create an index of prey abundance for the site and year in which a snake was captured. Modeling prey bycatch using a hierarchical model allows us to share information among trap lines and years to better infer the abundance of prey for sites and years in which the sample size is small. The zero-inflated Poisson model was fit in the Bayesian statistical software Stan using the "rstan" package (Carpenter and others, 2017) in $\mathrm{R}$ (version 3.3.1; R Core Team, 2016). The three prey abundances (fish, frogs, and tadpoles) were included as covariates of the growth coefficient $(k)$ in our von Bertalanffy growth model. We centered and standardized all covariates to have a mean of zero and unit standard deviation. If more than 1 year passed between captures of a snake, the covariate was averaged over the intervening years.

\section{Growth Analysis}

To model the growth of giant gartersnakes, we used a von Bertalanffy growth function (Fabens, 1965), which fits the indeterminate growth of reptiles well (Andrews, 1982).

$$
L_{t}=L_{t-1}+\left(L_{\infty}-L_{t-1}\right)\left(1-e^{-k \Delta t}\right)
$$

The von Bertalanffy growth function (eq. 1) is defined by two parameters, the asymptotic length $\left(L_{\infty}\right)$ individuals grow towards as they age and the growth coefficient, $k$, which defines how rapidly individuals approach $L_{\infty}$. We calculated $\Delta t$ as the number of days between captures, including the inactive season, to average growth over a 365-day year. Two models were fit on the basis of the von Bertalanffy growth function (eq. 1) to snake SVL data. The first model was a von Bertalanffy growth function that simultaneously models heterogeneity in growth by using a non-decreasing, stochastic Gamma process to model growth increments and measurement error by treating "true" SVL as a latent (unobserved) parameter (Eaton and Link, 2011; hereafter the "EL" model). Accounting for measurement error is important for snakes, as individuals may appear to "shrink" between captures (Madsen and Shine, 2001; Luiselli, 2005). A key component of the EL model is the $\lambda$ parameter, which is the ratio of the mean to the variance for the Gamma distribution used to model growth increments. A large value of $\lambda$ corresponds to a low level of individual heterogeneity in growth (Eaton and Link, 2011). We fit the EL model to a dataset with all growth increments from 1995 to 2016, including apparent negative growth increments because this provides data to estimate measurement error. Additive random effects of year and site on the mean growth coefficient, $k$, and site on $L_{\infty}$ were included. A random effect of individual snake on either $k$ or $L_{\infty}$ in the EL model was not included, instead the Gamma process was relied on to model heterogeneity among individuals (Eaton and Link, 2011). We included only growth intervals that encompassed at least one inactive season to ensure growth rates were comparable among individuals. From the von Bertalanffy function, $L_{\infty}$ and $k$ were estimated, 
which represent the way in which individuals grow to approach their asymptotic length. Because data on environmental covariates were available only from 2003 onwards and the EL model failed to converge when covariates were included, we fit a second, standard von Bertalanffy growth model (which confounds measurement error and individual variability in growth) to a second dataset that included environmental covariates but was limited to growth increments from 2003 to 2016. The standard von Bertalanffy model included random effects of site and individual on $L_{\infty}$, and site, year, and individual on $k$, but did not account for measurement error.

We fit von Bertalanffy growth curves using a Bayesian approach with Just Another Gibbs Sampler (JAGS) version 4.2 (Plummer, 2003), accessed through R (version 3.3.1; R Core Team, 2016) using the "runjags" (Denwood, 2015) and "R2jags" (Su and Yajima, 2015) packages for the EL model and standard models, respectively. We modified code from Eaton and Link (2011) to fit von Bertalanffy growth curves with measurement error and created the standard model on the basis of Fabens (1965). The EL model was run on 10 independent chains for 77,000 iterations each, with the first 7,000 iterations (before the chains converged on a stationary posterior distribution) discarded as burn-in, resulting in 700,000 iterations. The standard von Bertalanffy model was run with environmental covariates on seven independent chains for $1,100,000$ iterations each, with the first 100,000 iterations discarded as burn-in and each chain thinned by a factor of 100, resulting in 70,000 total iterations. We used uninformative or weakly informative prior distributions for all parameters of interest (table 2). The EL model (Eaton and Link, 2011) required narrower priors for mean $k$ and fixed and random effects on $k$ than those of the standard model in order to run and converge on a stationary posterior distribution. These priors were still uninformative for estimating $k$, given that the prior on $k$ for females is a uniform distribution from 0 to 0.01 , and the upper limit of the 95-percent credible interval of our estimate of $k$ was 0.00181 . Thus, the prior did not constrain the model's estimates of $k$. We used uniform priors for the standard deviation of random effects in the EL model to achieve convergence. In contrast, the standard model was more efficient using half-Cauchy priors than uniform priors for random effects. We evaluated the significance of environmental covariates by calculating the posterior probability that the covariate effect had the same sign as the median estimate. For all models, we inspected trace plots to ensure mixing of chains and evaluated model convergence using the Gelman-Rubin convergence diagnostic, $\hat{R}$ (Brooks and Gelman, 1998); all parameters had $\hat{R}$ greater than 1.05. We present posterior medians and symmetric 95 percent credible intervals for parameter estimates. 
Table 2. Prior distributions for growth model parameters for giant gartersnakes in Sacramento Valley, California.

[The values for $L_{\infty}, k$, and $\lambda$ represent the estimates for females, whereas the sex effect (for example, $\mathrm{m}_{k}$ ) represents the difference between the male estimate and the female estimate. The posterior probability represents the probability that a covariate has the same sign as the median estimate. EL, Eaton and Link model. SD, standard deviation]

\begin{tabular}{|c|c|c|c|c|c|c|c|}
\hline \multirow{2}{*}{ Model } & \multirow{2}{*}{ Parameter } & \multirow{2}{*}{ Symbol } & \multirow{2}{*}{ Prior distribution } & \multirow{2}{*}{ Median } & \multicolumn{2}{|c|}{ Percentile } & \multirow{2}{*}{$\begin{array}{l}\text { Posterior } \\
\text { probability }\end{array}$} \\
\hline & & & & & 2.5 & 97.5 & \\
\hline \multirow[t]{10}{*}{ EL } & Mean asymptotic size (mm) & $\mathrm{L}_{\infty}$ & Normal $(1000,100)$ & 910 & 865 & 954 & -- \\
\hline & Mean growth coefficient & $k$ & Uniform $(0,0.01)$ & 0.00129 & 0.00083 & 0.00181 & - \\
\hline & Individual variability in growth & $\lambda$ & Uniform $(0,100)$ & 12.7 & 9.4 & 16.9 & - \\
\hline & SD of year on $k$ & $\sigma_{\mathrm{ky}}$ & Uniform $(0,0.01)$ & 0.00042 & 0.00025 & 0.00069 & - \\
\hline & SD of site on $k$ & $\sigma_{\mathrm{ks}}$ & Uniform $(0,0.01)$ & 0.00040 & 0.00014 & 0.00119 & - \\
\hline & $\mathrm{SD}$ of site on $\mathrm{L}_{\infty}$ & $\sigma_{\mathrm{L}}$ & Uniform $(0,200)$ & 33 & 12 & 89 & -- \\
\hline & SD of measurement error & $\sigma_{\mathrm{e}}$ & $\operatorname{Gamma}(0.001,0.001)$ & 16 & 13 & 18 & - \\
\hline & Sex effect on $\mathrm{L}_{\infty}(\mathrm{mm})$ & $\mathrm{m}_{\mathrm{L}}$ & $\operatorname{Normal}(0,316)$ & -220 & -253 & -190 & -- \\
\hline & Sex effect on $k$ & $\mathrm{~m}_{\mathrm{k}}$ & $\operatorname{Normal}(0,0.01)$ & 0.00037 & 0.00006 & 0.00074 & 0.99 \\
\hline & Sex effect on $\lambda$ & $\mathrm{m}_{\lambda}$ & $\operatorname{Normal}(0,100)$ & 10.0 & 1.7 & 21.8 & 0.99 \\
\hline \multirow[t]{15}{*}{ Std } & Mean Asymptotic size (mm) & $\mathrm{L}_{\infty}$ & Normal $(1000,100)$ & 918 & 879 & 956 & - \\
\hline & Growth coefficient & $k$ & $\operatorname{Normal}(0,100)$ & 0.00106 & 0.00071 & 0.00144 & -- \\
\hline & SD of year on $k$ & $\sigma_{\mathrm{ky}}$ & Half-Cauchy $(0,1)$ & 0.00024 & 0.00003 & 0.00067 & - \\
\hline & SD of site on $k$ & $\sigma_{\mathrm{ks}}$ & Half-Cauchy $(0,1)$ & 0.00016 & 0.00001 & 0.00066 & - \\
\hline & SD of individual on $k$ & $\sigma_{\mathrm{ki}}$ & Half-Cauchy $(0,1)$ & 0.00053 & 0.00041 & 0.00057 & -- \\
\hline & $\mathrm{SD}$ of site on $\mathrm{L}_{\infty}$ & $\sigma_{\mathrm{Ls}}$ & Half-Cauchy $(0,1)$ & 29.47 & 12.49 & 72.86 & -- \\
\hline & $\mathrm{SD}$ of individual on $\mathrm{L}_{\infty}$ & $\sigma_{\mathrm{Li}}$ & Half-Cauchy $(0,1)$ & 1.2 & 0.04 & 32 & - \\
\hline & SD of growth increment & $\sigma_{\mathrm{g}}$ & Half-Cauchy $(0,1)$ & 27 & 23 & 32 & - \\
\hline & Sex effect on $\mathrm{L}_{\infty}(\mathrm{mm})$ & $\mathrm{m}_{\mathrm{L}}$ & $\operatorname{Normal}(0,100)$ & -221 & -248 & -194 & -- \\
\hline & Sex effect on $k$ & $\mathrm{~m}_{\mathrm{k}}$ & $\operatorname{Normal}(0,100)$ & 0.00044 & 0.00001 & 0.00081 & 0.99 \\
\hline & Effect of precipitation on $k$ & $\beta_{\mathrm{ppt}}$ & $\operatorname{Normal}(0,100)$ & 0.00018 & -0.00003 & 0.00035 & 0.96 \\
\hline & Effect of growing degree days on $k$ & $\beta_{\text {gdd }}$ & $\operatorname{Normal}(0,100)$ & 0.00000 & -0.00029 & 0.00019 & 0.49 \\
\hline & Effect of fish abundance on $k$ & $\beta_{\text {fish }}$ & $\operatorname{Normal}(0,100)$ & -0.00013 & -0.00030 & 0.00004 & 0.94 \\
\hline & Effect of frog abundance on $k$ & $\beta_{\text {frog }}$ & $\operatorname{Normal}(0,100)$ & 0.00017 & -0.00001 & 0.00036 & 0.96 \\
\hline & Effect of tadpole abundance on $k$ & $\beta_{\mathrm{tad}}$ & $\operatorname{Normal}(0,100)$ & 0.00008 & -0.00005 & 0.00020 & 0.89 \\
\hline
\end{tabular}




\section{Results}

We obtained 538 growth increments from 433 snakes from 1995 through 2016 (267 females, 166 males). For most snakes (351), we measured a single growth increment, although 62 snakes had two growth increments, 17 snakes had three growth increments, and three snakes had four growth increments. The largest number of samples were obtained from Colusa NWR, Gilsizer Slough, and the Natomas Basin, and few samples were obtained from Butte Farm 1, Butte Farm 2, and Sacramento NWR (table 1). The time intervals between captures varied from 160 to 3,580 days $(\bar{x}=448$ days), and growth increments varied between -40 and 492 millimeters (mm).

Female giant gartersnakes reached greater lengths than males. The estimated mean asymptotic $\operatorname{SVL}\left(L_{\infty}\right)$ for females was $910 \mathrm{~mm}$ (95-percent credible interval [CRI] $\left.=865-954 \mathrm{~mm}\right)$, whereas the estimated male mean $L_{\infty}$ was $690 \mathrm{~mm}(650-730 \mathrm{~mm})$ (fig. 2). The largest female measured 1,120 $\mathrm{mm}$ SVL, and the largest male was $790 \mathrm{~mm}$ SVL. The amount of individual heterogeneity in growth $(\lambda)$ was also sex dependent, with greater heterogeneity (smaller $\lambda$ ) among females than males (table 2 ). Males approached their asymptotic length faster $\left(k=1.67 \times 10^{-3} ; 1.13 \times 10^{-3}-2.26 \times 10^{-3}\right)$ than females $\left(k=1.29 \times 10^{-3} ; 8.3 \times 10^{-4}-1.81 \times 10^{-3}\right)$. Consequently, growth plateaued at a younger age for males than females (fig. 2). Most growth increments came from snakes between 400 and 1,000 mm SVL; few measurements came from larger or smaller individuals (fig. 2).

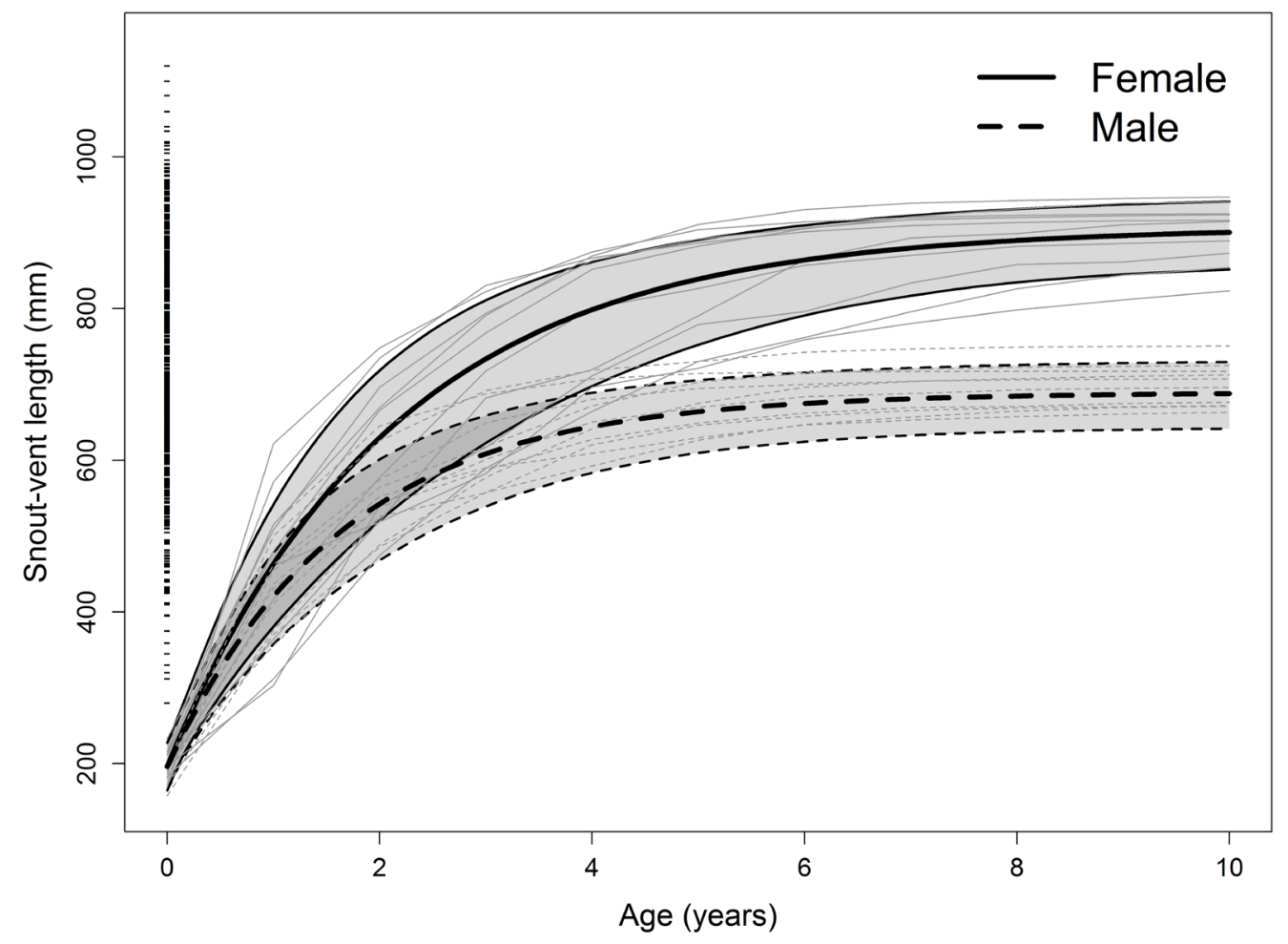

Figure 2. Growth curves for an average female (solid line) and male (dashed line) giant gartersnakes in the Sacramento Valley, California. Thick black lines represent median growth curves; thin black lines represent growth curves from 95-percent credible intervals. Gray lines are 10 randomly generated growth trajectories per sex to show individual variation. Dashes along the $y$-axis represent the size of individuals from which growth was measured. ( $\mathrm{mm}$, millimeter) 
Growth varied over time and among sites. Variation in growth among years (standard deviation of $\left.k=4.2 \times 10^{-4} ; 2.3 \times 10^{-4}-6.6 \times 10^{-4}\right)$ was similar to the variation among sites $\left(4.0 \times 10^{-4} ; 8.1 \times 10^{-5}-\right.$ $9.9 \times 10^{-4}$ ). The standard deviation (SD) of growth among sites and years amounts to approximately 30 percent of the average value of $k$. Snakes grew fastest at Gilsizer Slough and slowest at Badger Creek (fig. 3). Growth was fastest in 2003 and 1999, and slowest in 2013, 2012, and 2010 (fig. 4).

Asymptotic length varied among sites (SD of $L_{\infty}=33 \mathrm{~mm} ; 8-78 \mathrm{~mm}$ ), but this variation was small compared to the median $L_{\infty}$ for females (3.6 percent) and males ( 4.8 percent). Snakes reached the largest sizes at Colusa NWR and the smallest sizes at Gilsizer Slough (fig. 5). The random effects of site on $k$ and $L_{\infty}$ were not correlated $(r=-0.002)$.

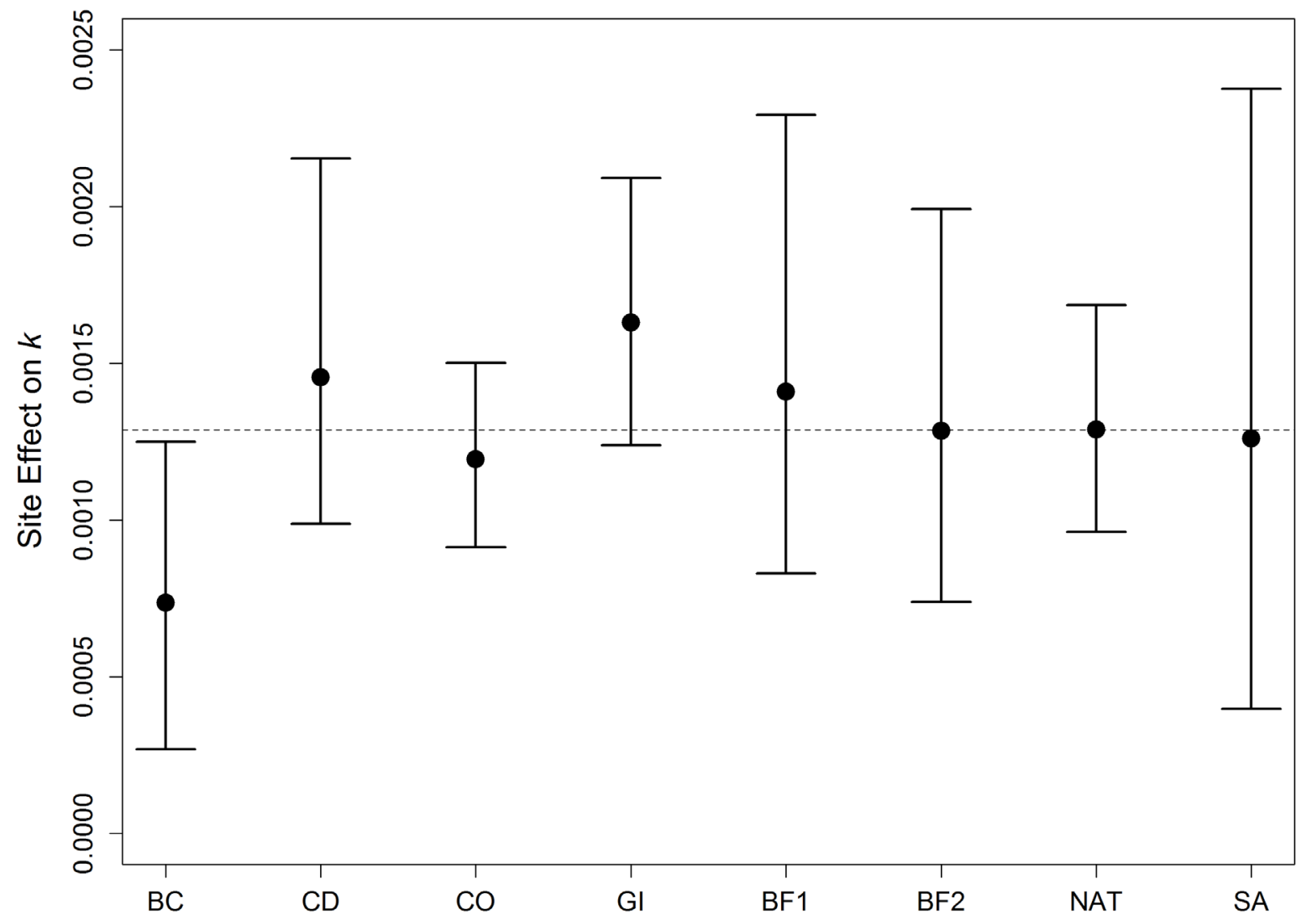

Figure 3. Effect of site on the von Bertalanffy growth coefficient, $k$, for giant gartersnakes at eight sites in the Sacramento Valley, California. Circles represent posterior median estimates of $k$ from each site; error bars show the 95-percent credible intervals. The horizontal dashed line represents the average estimate of $k$. Abbreviations for site names match codes in table 1. 


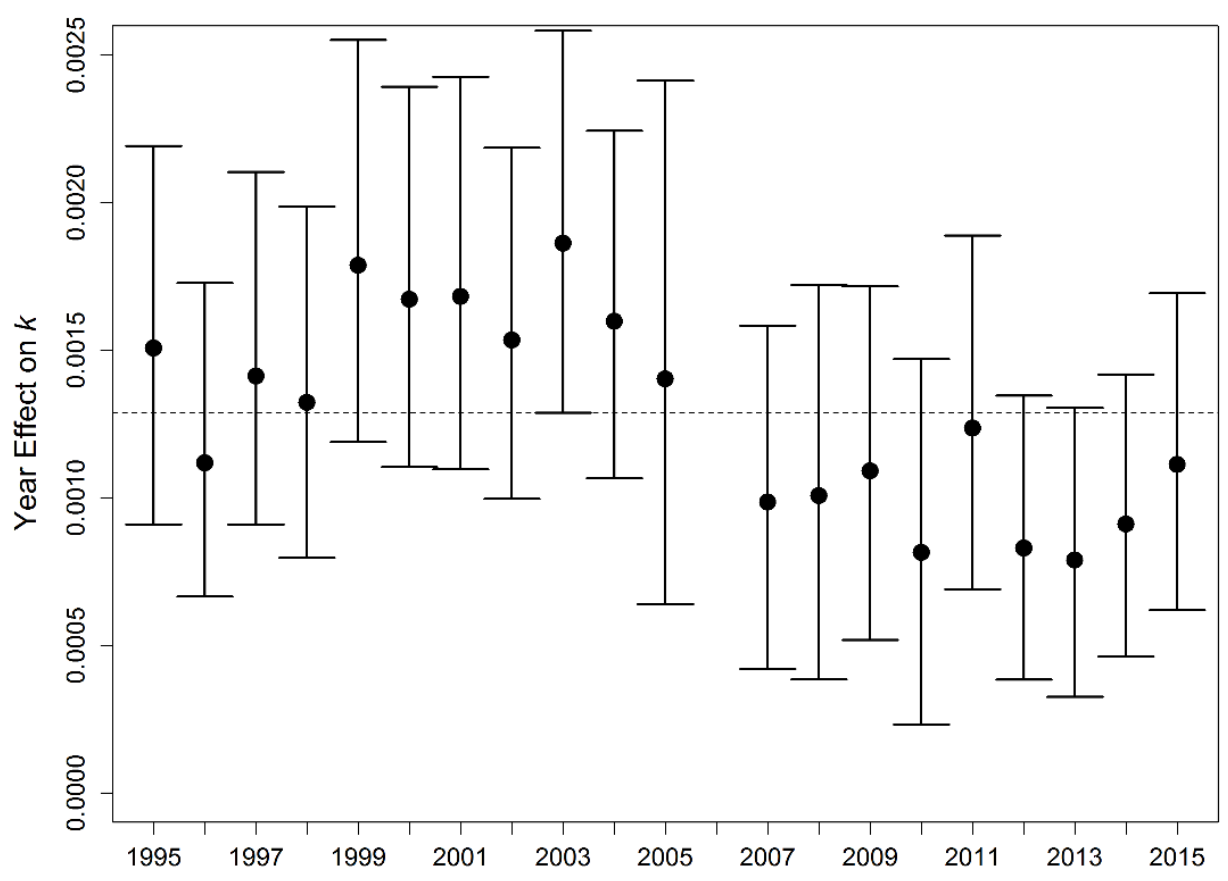

Figure 4. Effect of year on the von Bertalanffy growth coefficient, $k$, for giant gartersnakes in the Sacramento Valley, California, 1995-2015. Circles represent posterior median estimates of $k$ for each year; error bars show the 95-percent credible intervals. The horizontal dashed line represents the average estimate of $k$. No data were available for 2006.

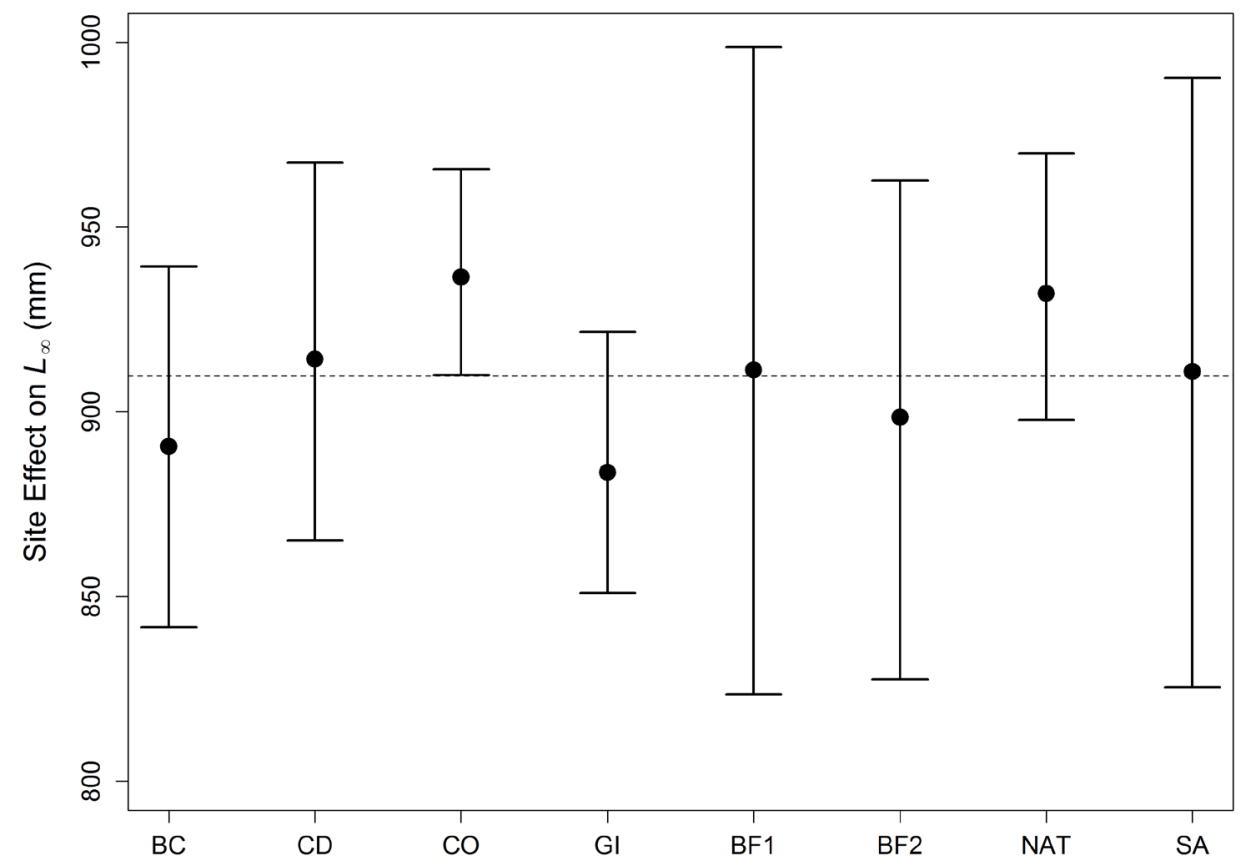

Figure 5. Effect of site on asymptotic length of giant gartersnakes in the Sacramento Valley, California. Circles represent posterior median estimates of asymptotic length from each site; error bars show the 95-percent credible intervals. The horizontal dashed line represents the average estimate of asymptotic length $\left(\mathrm{L}_{\infty}\right)$. Abbreviations for site names match codes in table 1. (mm millimeter) 
There was evidence of substantial error when measuring snake length. Approximately 10 percent (54/538) of growth increments were negative, and the average negative growth increment was $-15 \mathrm{~mm}$. This corresponds closely to the estimated measurement error parameter of the EL growth model. The standard deviation of measurement error was $16 \mathrm{~mm}(13-18 \mathrm{~mm})$, which is 2 percent of SVL averaged among females (780 mm SVL) and 3 percent of SVL averaged among males $(620 \mathrm{~mm}$ SVL).

Restricting the analysis to data from 2003 to 2016, the period for which we had data on climatic conditions and prey abundance, resulted in 305 growth increments from 254 snakes. A positive relationship between precipitation and snake growth was evident; increasing precipitation by one SD would increase $k$ by 16.6 and 11.7 percent for females and males, respectively. No evidence was found for a relationship between snake growth and the number of growing degree days in a year. The abundance of frogs at a site in a given year was positively related to growth (increasing frog abundance by 1 SD would result in a 15.6 and 11.1 percent increase of $k$ for female and males, respectively), but the abundance of fish was negatively related to growth (-12.4 and -8.8 percent). There was weaker evidence of a positive relationship between tadpole abundance and growth (7.4 and 5.3 percent; table 2).

The abundances of fish and frogs at a site in a given year were positively correlated $(r=0.68)$; all other predictors were weakly correlated $(r<0.36)$. To test for an interactive effect of fish and frog abundance on giant gartersnake growth, we fit a standard von Bertalanffy model that included a fish $\mathrm{x}$ frog interaction term. There was strong support for a negative fish $\mathrm{x}$ frog interaction $\left(\beta_{\text {fish } \mathrm{x} \text { frog }}=-1.2 \mathrm{x}\right.$ $10^{-4},-2.4 \times 10^{-4}$ to $-5.3 \times 10^{-6}$; posterior probability $\left.=0.98\right)$. When the model included a fish $\mathrm{x}$ frog interaction, the positive relationship between $k$ and frog abundance became weaker as the abundance of fish increased (fig. 6a-c). When frog abundance was high, fish abundance was negatively related to $k$, but fish abundance was positively related to $k$ when frog abundance was low (fig. $6 \mathrm{~d}-\mathrm{f}$ ).

The smallest sexually mature female giant gartersnake we recorded, based on the presence of embryos in an X-ray, was $541 \mathrm{~mm}$ SVL (section 2). Given our growth curve and the distribution of length at birth $(X=196 \mathrm{~mm} \mathrm{SVL}, \mathrm{SD}=16)$, on average, females would reach this minimum size for sexual maturity at 2 years old (55 percent of females). Because giant gartersnakes mate in the spring and give birth in late summer, an average female would produce offspring for the first time at 3 years old. Variation in growth among individuals means some snakes will not reach this size until they are greater than or equal to 3 years old ( 27 percent of females) and thus reproduce for the first time in their fourth year or later. However, some females that are large at birth and fast growing could reach this size threshold earlier and reproduce at 2 years old (18 percent of females). Less is known about the size at sexual maturity in male giant gartersnakes. Shine (1994) reports that for genera without malemale combat, male snakes reach sexual maturity at 79 percent of mean adult body length on average. Male giant gartersnakes would then be expected to mature at about $490 \mathrm{~mm}$ SVL. On average, they would reach this size at 2 years old and reproduce in the spring of their third year. 

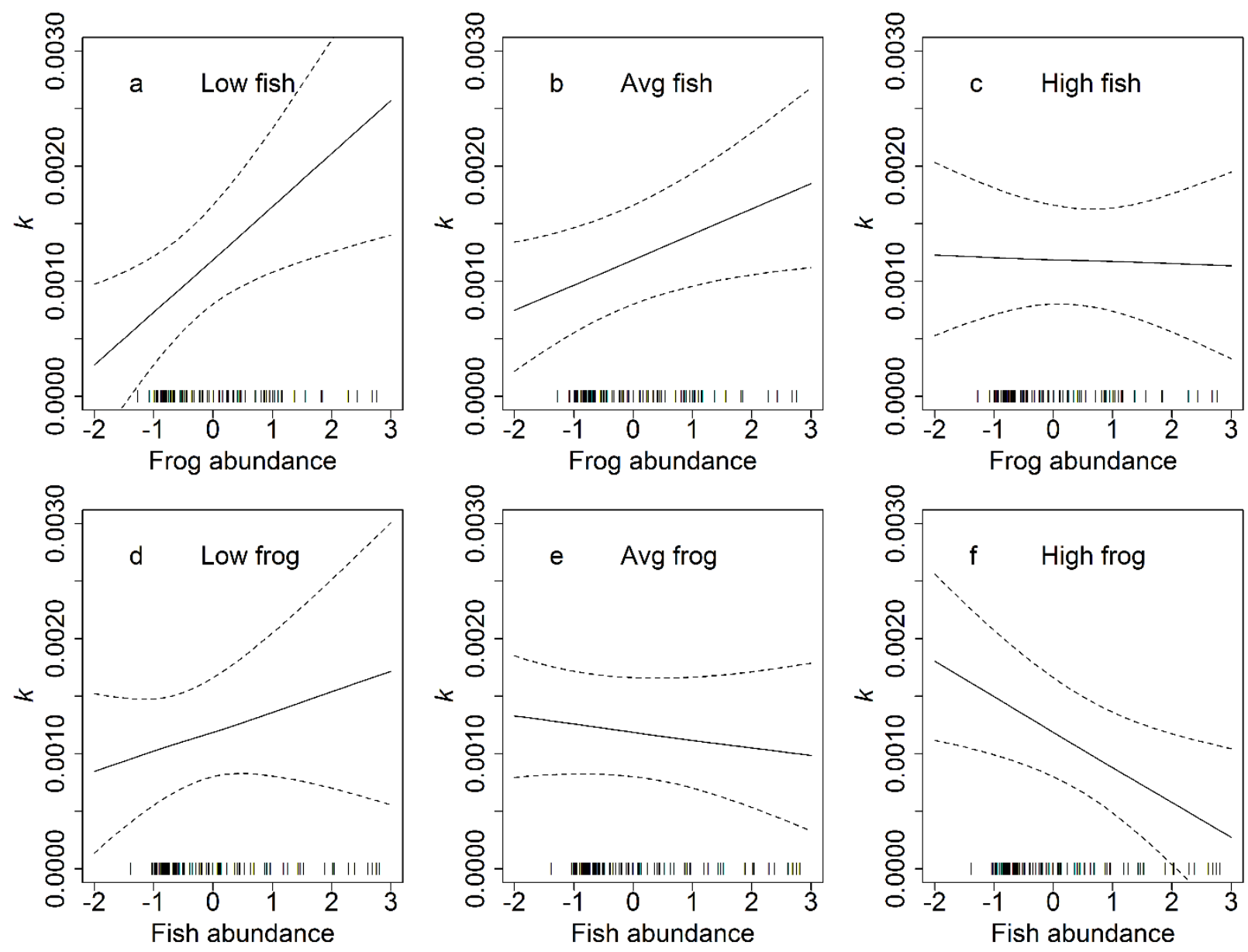

Figure 6. Interaction between fish and frog abundance and the growth coefficient, $k$ : Panels $a, b$, and $c$ plot $k$ in relation to standardized frog abundance, for low (-2), average (0), and high (2) levels of standardized fish abundance, respectively; Panels $\mathrm{d}, \mathrm{e}$, and $\mathrm{f}$ plot $k$ in relation to standardized fish abundance for low (-2), average $(0)$, and high (2) levels of standardized frog abundance, respectively, in the study area in California. Solid lines represent posterior median estimates, and dashed lines represent 95-percent credible intervals. Dashes along the $\mathrm{x}$-axis represent standardized fish and frog abundance values from study sites. (Avg, average) 


\section{Discussion}

The growth of giant gartersnakes varies among populations and over time. The asymptotic length of giant gartersnakes differed among sites, and snakes grew towards their asymptotic length more rapidly at some sites than others. Some of this variation may be explained by environmental factors. The growth of giant gartersnakes was positively related to the amount of precipitation and the abundance of frogs at a site and negatively related to the abundance of fish, with evidence of an interaction between the effects of fish and frog abundance. Giant gartersnakes also exhibit sexual size dimorphism, with males achieving smaller maximum sizes but approaching their maximum size at a faster rate than females. Our results reveal plasticity of growth in giant gartersnakes, which has implications for future demographic models of this threatened species. Because most of our data are from three sites, more widespread, long-term sampling is needed to fully measure the variation in growth patterns among giant gartersnake populations.

Despite their larger maximum size, the growth curves of giant gartersnakes indicate a life history similar to that of other gartersnakes, although female giant gartersnakes may take a year longer to reach sexual maturity. We estimated that on average, female giant gartersnakes first reproduce at 3 years old. We assumed a fixed size at which females reach maturity, but in reality there is likely variation among individuals and populations in this key life-history characteristic; therefore, individuals might mature at a younger or older age (Bernardo, 1993). Most studies of growth and maturity in gartersnakes are from populations at higher latitudes than the present study area, where snakes presumably have shorter active seasons. Carpenter (1952) concluded that female T. butleri (Butler's gartersnake), T. sauritus (ribbon snake), and T. sirtalis (common gartersnake) in Michigan, USA, can reach maturity at 2 years old. Female T. radix (plains gartersnake) in Illinois, USA, and Alberta, Canada, reach asymptotic lengths of 582 and $757 \mathrm{~mm} \mathrm{SVL}$, respectively, and first reproduce at 2 or 3 years old on average (Stanford and King, 2004; Tuttle and Gregory, 2012). Likewise, female T. sirtalis in Kansas can reach maximum lengths of $950 \mathrm{~mm}$ and first reproduce at 2 years old (Fitch, 1965). Among new world Natricinae, the asymptotic length of giant gartersnakes is most similar to Nerodia sipedon (common watersnake). Male $N$. sipedon sipedon (northern watersnake) in Ontario, Canada, and $N$. sipedon insularum (Lake Erie watersnake) in Ohio approach $620 \mathrm{~mm}$ and $714 \mathrm{~mm}$ SVL, respectively; females approach $920 \mathrm{~mm}$ and $1,015 \mathrm{~mm}$ and reach sexual maturity at 4 years old on average (Brown and Weatherhead, 1999a, 1999b; King and others, 2016). The warmer climate and longer active season in central California could allow giant gartersnakes to reach comparable sizes in a shorter time than populations of $N$. sipedon in colder climates. Life histories can be variable within a species of Thamnophis (Miller and others, 2011), and the faster growth and smaller asymptotic length of snakes at Gilsizer Slough hints at the potential for life-history differences among giant gartersnake populations. More data on spatial variation in the size and age at sexual maturity are needed to determine whether giant gartersnake populations exhibit different life histories. 
The growth of giant gartersnakes might not be expected to be affected by local precipitation, given snakes largely occupy habitats supplied by managed water deliveries during the summer. The productivity of a site may depend on precipitation, however. Rainfall stimulates amphibian breeding activity (Todd and Winne, 2006), with wetter years producing greater reproductive output (Jensen and others, 2003). Thus, higher precipitation may increase the abundance of amphibian prey available for giant gartersnakes. Greater precipitation also could increase the productivity of insects that support populations of giant gartersnake prey (Batzer and Wissinger, 1996). Years with higher precipitation might also make more water available to grow rice and increase the available area of wetland-like habitat. The importance of precipitation to giant gartersnake growth is further supported by the slower growth from 2012 to 2015 during an extreme drought (Robeson, 2015). Altered precipitation patterns in California because of climate change (Hayhoe and others, 2004) could affect giant gartersnake growth.

The contrasting effects of frog and fish abundance on giant gartersnake growth and the evidence for a fish-frog interaction point to the complexity of interspecific interactions in wetland communities. In general, greater abundance of frogs at a site was related to faster growth, and greater abundance of fish was related to slower growth, but in each case the effect of one prey type was conditional on the abundance of the other. Snakes may grow faster when frogs are more abundant because giant gartersnakes prefer frogs over other prey (Ersan, 2015), but this positive relationship was attenuated at high levels of fish abundance. In contrast, there was a negative relationship between fish abundance and giant gartersnake growth when frog abundance was high but a positive relationship between fish abundance and giant gartersnake growth when frog abundance was low. One potential explanation for this interaction is that when frog abundance is low, fish are the only available prey; thus, increased fish abundance provides more food. When frog abundance is high, however, increasing fish abundance might lead to competition between fish and giant gartersnakes for anuran prey (frog or toad). For example, the presence of fish reduces the survival of native Pseudacris regilla (Pacific treefrogs; Adams, 2000). Our simple index of fish abundance does not account for the size or type of fish present, and different fish species could serve as prey, predators, or competitors for giant gartersnakes. Future studies could investigate how fish species composition affects the growth of giant gartersnakes.

Growth data are necessary for demographic models used to design and evaluate management efforts for wildlife. The spatial and temporal variability in growth seen in many reptiles, including giant gartersnakes, demonstrates that researchers need to be cautious about drawing conclusions on the basis of short-term studies, or extrapolating data from one population to infer the status of another. The sensitivity of population growth to changes in vital rates can vary among populations of the same species (Miller and others, 2011). Thus, management based on information from a population with fast growth and smaller adult body sizes, might not be optimal for a population with slower growth and larger adult size. Likewise, data collected over a short period are unlikely to encapsulate the natural variance in vital rates, such as growth (Beissinger and Westphal, 1998). For example, if population projections are based on growth during years with a favorable climate, managers might adopt an overly optimistic outlook for the future of an imperiled species. Demographic models for threatened reptiles, such as giant gartersnakes, can provide sound tools for conservation and management when they include spatial and temporal variation in growth. Our results reinforce the rationale for long-term, multi-site studies of growth among reptile populations. Ignoring spatial and temporal variation in growth could be detrimental to the conservation and management of an imperiled species. 


\section{Section 2: Reproductive Frequency and Size-Dependence of Fecundity in the Giant Gartersnake}

\section{Introduction}

The age or size at which individuals reach maturity, the frequency of reproduction, and the manner in which fecundity changes with age and size are fundamental life-history traits. In snakes, fecundity varies among closely related species (Rossman and others, 1996; Gibbons and Dorcas, 2004), among populations within species (Bronikowski and Arnold, 1999), and among individuals within a population (Aldridge and others, 1995; Weatherhead and others, 1999). In natricine colubrids there is generally a positive relationship between litter size and maternal size, whereas relationships between neonate size, litter size, and maternal size are more complex (Ford and Killebrew, 1983; Ford and Karges, 1987; King, 1993; Weatherhead and others, 1999). Within a species, there can be variation in the timing of reproduction within individuals' lives and the amount of energy invested into individual litters. For example, nearby populations of Thamnophis elegans (western terrestrial gartersnake) in different habitats differ in their growth rates, age at maturity, fecundity, and even whether fecundity is related to maternal size (Bronikowski and Arnold, 1999). Populations of $T$. sirtalis (common gartersnake) in Canada exhibit substantial variability in reproductive characteristics, such as litter size and average neonate size (Gregory and Larsen, 1993). There can also be inter-annual variation in female fecundity within a population (Seigel and Fitch, 1985). Because of this interspecific and intraspecific variation in reproductive characteristics, it may be inappropriate to make assumptions about the life history of a poorly studied species on the basis of knowledge from closely related species.

Although Thamnophis have often been the subject of studies of snake reproductive biology, little is known about reproduction in the giant gartersnake, T. gigas. Previous studies of the reproductive biology of giant gartersnakes have been limited by small sample sizes and did not investigate (Hansen and Hansen, 1990) or find evidence of (Halstead and others, 2011b) sizedependent fecundity. Giant gartersnakes may be expected to differ in reproductive biology and life history from other Thamnophis because of their larger size, and because giant gartersnakes inhabit a much warmer climate than populations of $T$. sirtalis and T. elegans whose reproductive biology and life history have been the subject of thorough studies (Gregory and Larsen, 1993; Bronikowski and Arnold, 1999). A better understanding of the reproductive biology and life history of giant gartersnakes is needed to inform effective conservation and management of this species.

We studied reproductive characteristics of female giant gartersnakes from six regions distributed throughout its range in the Sacramento Valley of California. X-radiography of adult females and litters born in captivity were used to measure litter size. We tested whether litter size and offspring size were related to maternal size. We also report variation in the proportion of females that are reproductive each year and the incidence of stillbirth in litters born in captivity. On the basis of studies of other Thamnophis, we predicted that larger females would produce larger litters. The results of this study contribute to the understanding of the reproductive biology of a threatened species, and fill a gap in the knowledge of the reproductive biology of species in the genus Thamnophis. 


\section{Methods}

\section{Data Collection}

Female giant gartersnakes were captured in six regions of the Sacramento Valley from 2013 to 2016 (fig. 7, table 3). Study sites in the Sutter Basin, Central Colusa Basin, and Natomas Basin regions include canals associated with rice agriculture and restored wetlands managed for giant gartersnakes and other aquatic wildlife. The habitats in the Southern Colusa Basin and Northern and Southern Butte Basins consist of canals adjacent to rice agriculture (fig. 7). Snakes were captured by hand and by using modified aquatic funnel traps (Casazza and others, 2000; Halstead and others, 2013) placed along the edges of wetlands or emergent vegetation. All captured snakes were uniquely marked by branding ventral scales (Winne and others, 2006) and inserting passive integrated transponder (PIT) tags. We determined the sex and measured the snout-vent-length (SVL) and mass of each snake. We took females to the Sacramento Zoo and performed X-radiography to count the number of ovulated eggs or developing embryos in the females' oviducts. We examined 73 individuals; 47 individuals were examined in only 1 year, 21 were examined in 2 years, and 5 were examined in 3 years, resulting in a total of 104 X-rays. Of the 73 snakes examined, 56 were part of a radio-telemetry study and previously had surgery to implant and remove radio transmitters. These 56 females were chosen because they were large enough to accommodate radio-transmitters, their putative reproductive status did not influence their inclusion in the study. Data from the 56 radio-telemetry females were used in the analysis of both litter size and reproductive status/frequency. The remaining 17 snakes that were Xrayed in 2016 were chosen because they appeared gravid when captured, and data from these snakes was only used for the analysis of litter size and its relationship to maternal size. To determine whether a female appeared gravid, we first palpated the abdomen in the field to feel for the presence of embryos or ovulated eggs. In addition to the X-rayed snakes, 13 gravid females were collected from the Central Colusa Basin ( $n=4)$, Sutter Basin $(n=4)$, and Natomas Basin $(n=5)$ from 2013 to 2014 and kept in captivity until parturition for a study on neonate prey preference (Ersan, 2015). After parturition, we counted the number of live and stillborn neonates in these captive-born litters and weighed and measured the SVL of all live neonates within 4 days of their birth. We also included records of litter size, maternal size, and neonate SVL from captive-born litters in two previous studies of reproduction of giant gartersnakes in some of our analyses described below (Hansen and Hansen, 1990; Halstead and others, 2011b). 
Table 3. Sampling period, number of individuals, and sources of litter size estimates for the giant gartersnakes in Sacramento Valley, California.

[Gravid refers to the number of X-rays in which the individuals were gravid. Individuals includes snakes that were X-rayed and snakes that gave birth to litters in captivity. NWR, National Wildlife Refuge]

\begin{tabular}{llccccl}
\hline \multicolumn{1}{c}{ Site } & \multicolumn{1}{c}{$\begin{array}{c}\text { Years } \\
\text { sampled }\end{array}$} & Individuals & X-rays & Gravid & $\begin{array}{c}\text { Captive } \\
\text { litters }\end{array}$ & Source \\
\hline Northern Butte Basin & $2015-2016$ & 7 & 9 & 6 & 0 & This study \\
Southern Butte Basin & $2015-2016$ & 6 & 7 & 5 & 0 & This study \\
Central Colusa Basin & $2014-2016$ & 20 & 24 & 9 & 4 & This study \\
Southern Colusa Basin & $2014-2016$ & 21 & 31 & 19 & 0 & This study \\
Sutter Basin & $2013-2016$ & 26 & 33 & 11 & 4 & This study \\
Natomas Basin & $2013-2014$ & 5 & 0 & 0 & 5 & This study \\
Badger Creek & 1997 & 2 & 0 & 0 & 2 & Halstead and others (2011b) \\
Colusa NWR & 1997 & 1 & 0 & 0 & 1 & Halstead and others (2011b) \\
Gilsizer Slough & $1995-1996$ & 6 & 0 & 0 & 6 & Halstead and others $(2011 \mathrm{~b})$ \\
Sacramento NWR & $1995-1997$ & 2 & 0 & 0 & 2 & Halstead and others $(2011 \mathrm{~b})$ \\
Fresno County & 1978 & 4 & 0 & 0 & 4 & Hansen and Hansen $(1990)$ \\
Sacramento County & $1987-1990$ & 12 & 0 & 0 & 12 & Hansen and Hansen $(1990)$ \\
\hline
\end{tabular}




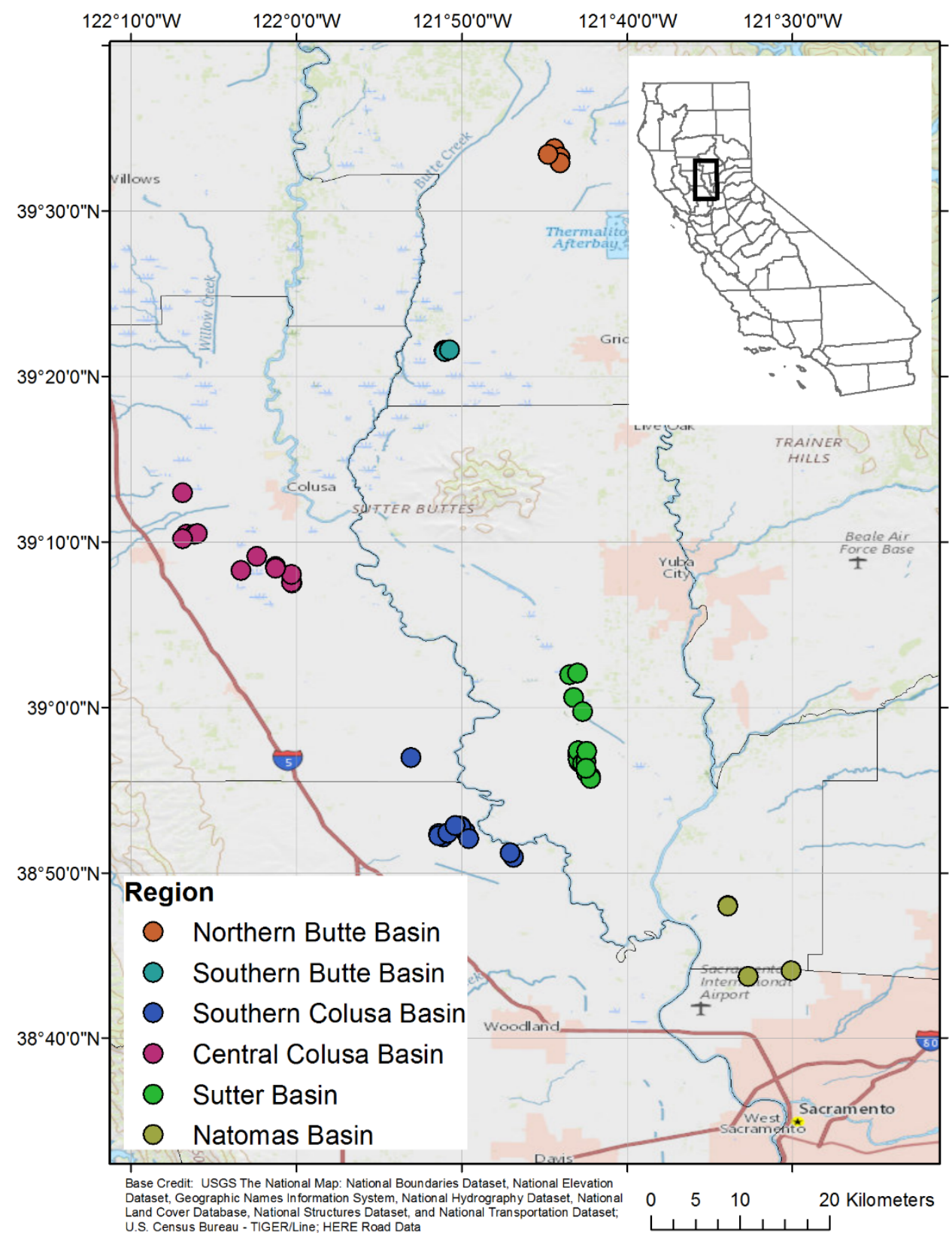

Figure 7. Location of study sites for giant gartersnake fecundity in the Sacramento Valley of California. 


\section{Statistical Analysis}

All of the fecundity data were analyzed using Bayesian hierarchical models. We modeled the number of neonates produced by a female (litter size) using a Poisson response with a log link function. Live and stillborn offspring were included in the measure of litter size, following the recommendations of Gregory and others (1992). Some of our fecundity data come from X-rays of gravid females, and we do not know how many of the eggs or embryos counted were carried to term and born alive. By including the sum of live and dead young from captive litters in the measure of litter size, we felt that litter size data from these two sources were comparable and could be pooled for some analyses. In addition, the number of live and stillborn offspring is likely a better measure of female investment into reproduction than live offspring alone. Because we had multiple sources of data on litter size and female SVL, we fit three separate models. First, we tested for a relationship between female SVL and litter size using X-rayed females and captive-born litters from the current study only with a fixed effect of data source on the intercept. Second, we fit a model to a pooled dataset that combined litters from the current study with litters born in captivity from 1995 to 1997 (Halstead and others, 2011b) and from 1978 to 1990 (Hansen and Hansen, 1990). Random effects of region, year, and individual on the intercept were included in all models to reflect the geographical clustering of regions, temporal variability, and repeated measures of fecundity of some snakes. Finally, we initially included a fixed effect of study on litter size as well, but preliminary models showed no difference in the intercept among studies, so our final model included no effect of study. To generate a model with parameters comparable to earlier studies of maternal size-fecundity relationships in natricine snakes (for example, Ford and Karges, 1987; Bronikowski and Arnold, 1999), we also fit a simple linear regression of litter size on maternal SVL without transforming either variable.

Relationships between neonate size (neonate SVL and mass as response variables) and maternal SVL and litter size (predictors) were tested by fitting a linear model to data from 12 captive litters in this study (1 litter was lacking morphology data from the sole live neonate) and 168 neonates from 11 captive litters reported by Halstead and others (2011b). The model included a fixed effect of study to model potential differences in neonate size between the two studies and a random effect of litter to account for the non-independence among neonates from the same litter. We tested for effects of maternal SVL and litter size on the probability that a litter contained stillborn offspring by pooling data from this study and from Halstead and others (2011b) and fitting a binomial model with random effects of site and year.

Using data from X-rays only, we estimated the proportion of females that were reproductive in a given year and the frequency of reproduction in individuals that were examined in more than 1 year. This analysis was limited to radio-telemetry females that were X-rayed from 2014 to 2016 during the "pre-parturition period" between 1 May, when females are likely to have enlarged eggs that are visible on radiographs, and 11 July, the earliest recorded date of parturition in giant gartersnakes (Halstead and others, 2011b). We did not include the 17 females that were X-rayed in July and August 2016, because these females were examined because they appeared gravid, and thus represent a biased sample. We fit a binomial model with a fixed effect of year on the proportion of females that were reproductive $(p)$ and random effects of site and individual. We tested for differences in the proportion of reproductive females each year by calculating the difference between the estimates of $p$ for each year. We also included a fixed effect of female SVL, and linear and quadratic effects of Julian date on $p$, to account for potential differences in the size of females and timing of examinations each year. 
For 12 females, we did not measure the SVL for 1 year in which they were captured and examined, but we had a measure of SVL from the previous year. The SVLs were imputed for the missing year using Bayesian imputation (Bonner and others, 2010) and a von Bertalanffy growth curve for T. gigas (section 1). This allowed us to include these individuals in our analysis while accounting for uncertainty in their size in years in which they were examined but not measured.

We analyzed all regression models using JAGS (Plummer, 2003), accessed through R version 3.3.1 (R Core Team, 2016) using the R2jags package (Su and Yajima, 2015). Uniform $(0,1)$ priors were used on all probability parameters, and a weakly informative $\operatorname{Normal}(\mu=0, \sigma=10)$ prior was used for the effect of maternal SVL on litter size, neonate SVL, and neonate mass. All covariates were standardized by subtracting the mean and dividing by the standard deviation to ease interpretation of model coefficients. Half-Cauchy priors were used for the standard deviation of random effects (Gelman, 2006). Models were run on 7 chains for 400,000 iterations after a burn-in of 100,000, and the resulting chains were thinned by a factor of 40 , resulting in a total of 70,000 iterations. We visually inspected trace plots to ensure chains were well mixed and evaluated convergence using the $\hat{R}$ statistic: all $\hat{R}$ values were less than 1.01 (Brooks and Gelman, 1998). To assess the evidence for a relationship between a covariate and the response variable, we measured the posterior probability that the coefficient for the covariate had the same sign as its median estimate. Unless otherwise noted, throughout the results in this section we present this posterior probability, along with posterior median estimates and 95-percent credible intervals (CRI) for all model parameters.

\section{Results}

X-rayed females ranged from 514 to 1,040 millimeters $(\mathrm{mm})$ SVL (mean $=790 \mathrm{~mm})$ and 99 815 grams $(\mathrm{g})$ mass (mean $=317 \mathrm{~g}$ ) (table 2). Of the $104 \mathrm{X}$-ray examinations, 50 snakes were gravid, and we counted the number of eggs or embryos. Gravid females were slightly shorter on average than non-gravid females $(\bar{x}=773 \mathrm{~mm} \mathrm{SVL}$ versus $\bar{x}=806 \mathrm{~mm} \mathrm{SVL}, \mathrm{p}$ (gravid<non-gravid) $=0.96$ ). The smallest gravid female was $541 \mathrm{~mm}$ SVL. The earliest a female was determined to be gravid from an X-ray was April 8, and the latest was September 1. The 13 females that gave birth to litters in captivity had parturition dates from July 12 to September 12. Females that gave birth in captivity were slightly shorter $(731.5 \mathrm{~mm})$ and lighter on average than the X-rayed females (table 4$)$.

Table 4. Characteristics of examined female giant gartersnakes and their litters, Sacramento Valley, California.

[SVL, snout to vent length; N, number of individuals; SD, standard deviation; N, number; mm, millimeter; g, gram]

\begin{tabular}{lrcrll}
\hline \multicolumn{1}{c}{ Parameter } & N & Mean & SD & \multicolumn{1}{c}{ Range } & \multicolumn{1}{c}{ Source } \\
\hline Maternal SVL (mm) & 104 & 790 & 88.6 & $514-1040$ & X-rays \\
& 13 & 731.5 & 90.5 & $640-910$ & Captive females \\
Maternal mass (g) & 104 & 317 & 114.3 & $99-815$ & X-rays \\
& 13 & 293.2 & 143.6 & $186-664$ & Captive females \\
Litter size & 50 & 15.9 & 4.6 & $8-33$ & X-rays \\
& 13 & 15.5 & 7.9 & $5-35$ & Captive litter (Total) \\
Neonate SVL (mm) & 13 & 11.8 & 6.3 & $1-23$ & Captive litter (Live) \\
Neonate mass (g) & 154 & 187.7 & 10.1 & $146-207$ & Captive litter (Live) \\
Date of parturition & 154 & 4.3 & 1 & $2-7.3$ & Captive litter (Live) \\
\hline
\end{tabular}




\section{Litter Size, Neonate Size, and Maternal Size}

Counts of embryos and ovulated eggs from X-rays had an average of 15.9 offspring (range 833). The 13 litters born in captivity had an average total litter size of 15.5 offspring (5-35), of which an average of 11.8 (1-23) individuals were born alive. Seven out of 13 litters had at least one dead neonate, and out of 202 neonates, 48 were stillborn. At least one litter from each of the three regions had dead neonates. The number of stillborn offspring in a litter ranged from 0 to 22, with an average of 3.7 stillborn offspring per litter. When we pooled data on stillbirth from this study with data from 11 captive-born litters from Halstead and others (2011b), 13 of 24 litters had at least one stillborn neonate. There was some evidence for a positive effect of maternal SVL on the probability that a litter contained at least one stillborn neonate in the pooled data, although the credible interval overlapped zero $\left(0.73,-0.40-2.01 ; \mathrm{p}\left(\beta_{\mathrm{SVL}}>0\right)=0.89\right)$. Litter size was not related to the incidence of stillbirth $\left(0.02,-0.12-0.17 ; \mathrm{p}\left(\beta_{\mathrm{Fec}}>0\right)=0.62\right)$.

There was slight variation in average litter size among regions. The Natomas Basin had the smallest average litter size with 12.2 offspring $(n=5)$, and the largest average litter size was 17.3 offspring $(\mathrm{n}=15)$ for snakes from the Sutter Basin. Average litter size was consistent among years, ranging from 15 offspring in $2013(n=3)$ to 16.9 in $2015(n=17)$. The variance in litter size among regions and years was minimal after accounting for maternal size (see next paragraph below).

There was a clear positive relationship between female SVL and litter size: longer females produced larger litters (table 5; fig. 8). There was no difference in the average litter size between Xrayed female litters and captive-born litters $\left(0.08,-0.12-0.27 ; \mathrm{p}\left(\alpha_{\mathrm{cap}}>\alpha_{\mathrm{X}-\text { ray }}\right)=0.79\right)$. After accounting for maternal SVL, the variation in litter size attributable to year, region, and individual were each less than 2 percent of the natural log of litter size for a female of average SVL (table 5). We also fit a model that pooled data from this study with data from 11 captive-born litters from 1995 to 1997 (Halstead and others, 2011b) and 16 captive-born litters from 1978 to 1990 (Hansen and Hansen, $1990)$, resulting in 90 total litters. The estimated intercept $\left(\alpha_{0}=2.79,2.70-2.89\right)$ and slope $\left(\beta_{\mathrm{SVL}}=\right.$ $0.22,0.17-0.27)$ of the size-fecundity relationship when all four data sources were pooled were nearly equal to the estimates from the model of the current study alone (table 5). When we fit a simple linear regression of litter size on untransformed maternal SVL, there was again a strong positive relationship $\left(\beta_{\mathrm{SVL}}=0.039,0.029-0.048\right)$.

Table 5. Parameter estimates from a Poisson regression model of the effect of maternal snout-vent length on litter size of the giant gartersnake, Sacramento Valley, California.

[Data include captive-born litters $\left(\alpha_{\text {cap }}, \mathrm{n}=13\right)$ and X-ray embryo counts $\left(\alpha_{X}\right.$-ray,$\left.n=50\right) . \%$, percent; SVL, snout-vent length, $\alpha_{\text {cap }}$ and $\alpha_{\mathrm{X} \text {-ray }}$ represent the intercepts for the captive-born litters and X-ray embryo counts respectively. $\beta_{\mathrm{SVL}}$ represents the slope of the litter-size maternal SVL relationship, and $\sigma_{\text {ind, }}, \sigma_{\text {site, }}$ and $\sigma_{\text {year }}$ represents the standard deviation of individual, site, and year random effects. All parameters are on the natural log scale]

\begin{tabular}{lccc}
\hline \multirow{2}{*}{ Parameter } & \multirow{2}{*}{ Median } & \multicolumn{2}{c}{$\mathbf{9 5 \%}$ credible interval } \\
\cline { 3 - 4 } & & $\mathbf{2 . 5 \%}$ & $\mathbf{9 7 . 5 \%}$ \\
\hline$\alpha_{\text {cap }}$ & 2.85 & 2.63 & 3.06 \\
$\alpha_{\mathrm{X}-\text {-ray }}$ & 2.77 & 2.58 & 2.93 \\
$\beta_{\text {SVL }}$ & 0.22 & 0.16 & 0.28 \\
$\sigma_{\text {ind }}$ & 0.046 & 0.0018 & 0.15 \\
$\sigma_{\text {site }}$ & 0.047 & 0.0021 & 0.21 \\
$\sigma_{\text {year }}$ & 0.054 & 0.0025 & 0.38 \\
\hline
\end{tabular}




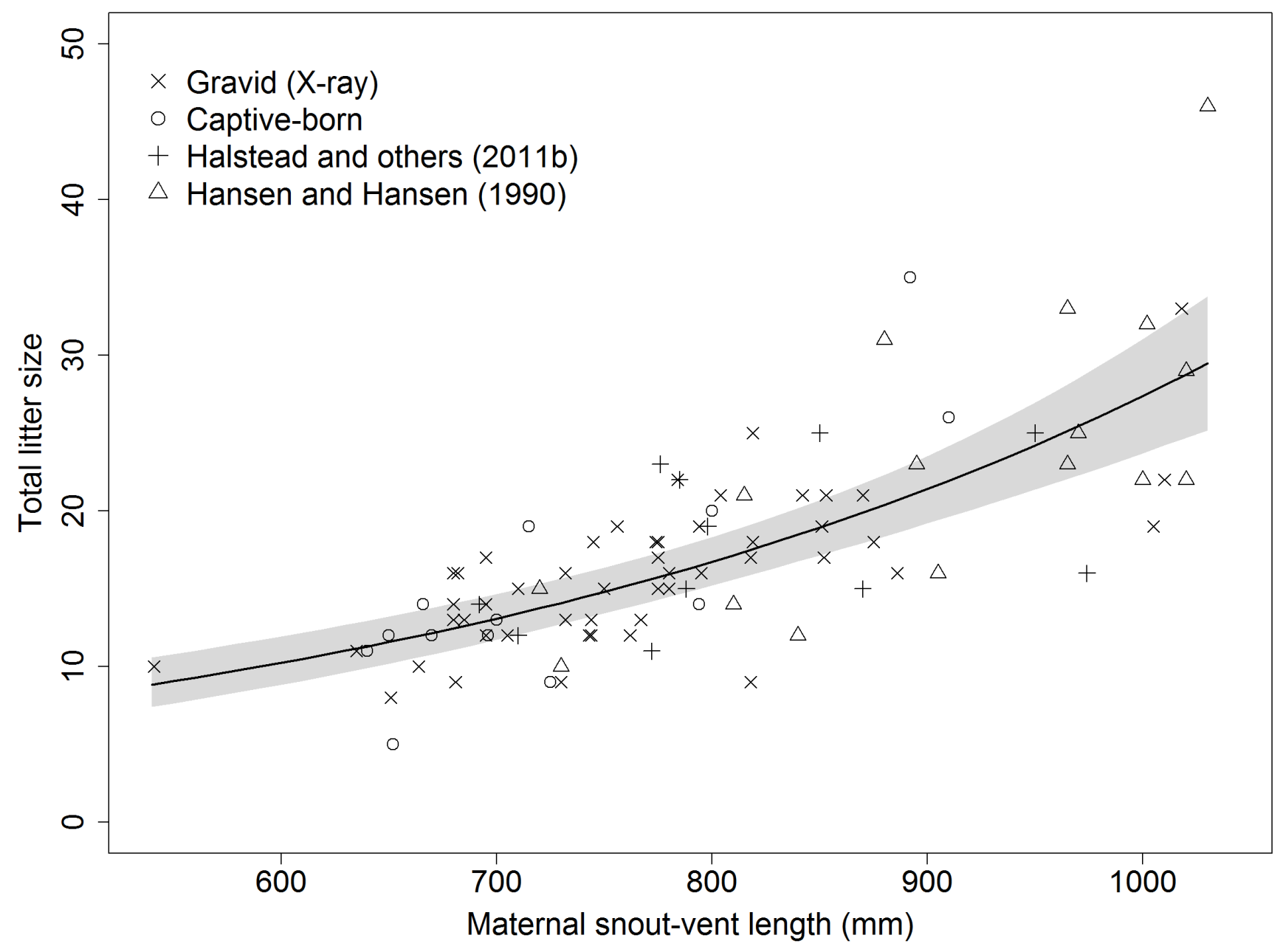

Figure 8. Maternal snout-vent length of the giant gartersnake in relation to litter size for data from the current study in Sacramento Valley, California (Hansen and Hansen, 1990; Halstead and others, 2011b). The black line represents the median relation, and the gray shading represents the 95-percent credible interval. (mm, millimeter.) 
Live neonates $(\mathrm{n}=154)$ averaged $187.7 \mathrm{~mm}$ SVL $(146-207)$ and $4.3 \mathrm{~g}$ mass $(2-7.3)$. The average SVL of neonates varied among litters from a low of $174.8 \mathrm{~mm}(\mathrm{n}=5)$ to a high of $199.3 \mathrm{~mm}$ $(\mathrm{n}=12)$. Neonates in the current study were shorter on average than neonates from Halstead and others (2011b) $(-15.9 \mathrm{~mm},-24.1$ to $-7.04 ; \mathrm{p}(\operatorname{diff}<0)=1)$, but average neonate mass did not differ between the two studies $(-0.19 \mathrm{~g},-0.99-0.59 ; \mathrm{p}(\operatorname{diff}<0)=0.69)$. There was some evidence that neonate size (mass and SVL) was negatively related to total litter size, although credible intervals overlapped zero. Similarly, there was support for a negative relationship between neonate mass and maternal SVL, but evidence of a positive relationship between neonate SVL and maternal SVL was weaker (fig. 9, table $6)$.

Table 6. Parameter estimates from a regression model of the effects of giant gartersnake maternal snout-vent length and litter size on neonate mass and snout-vent length, Sacramento Valley, California.

[Data are captive-born litters from this study $\left(\alpha_{R}, n=12\right)$ and Halstead and others $(2011 b)\left(\alpha_{H}, n=11\right)$. $\beta_{F e c}$ represents the effect of litter size on neonate size, and $\beta_{\mathrm{SVL}}$ represents the effect of maternal SVL on neonate size. $\sigma_{\text {mass }}$ and $\sigma_{\mathrm{SVL}}$ are the variation in mass and snout-vent length (SVL) among neonates, whereas $\sigma_{\text {litter }}$ represents the random effect of litter on neonate SVL and mass]

\begin{tabular}{clrrr}
\hline \multirow{2}{*}{ Response } & \multirow{2}{*}{ Parameter } & \multirow{2}{*}{ Median } & \multicolumn{2}{c}{$95 \%$ credible interval } \\
\cline { 4 - 5 } & & & $\mathbf{2 . 5 \%}$ & $\mathbf{9 7 . 5 \%}$ \\
\hline Neonate mass & $\alpha_{\mathrm{R}}$ & 4.31 & 3.75 & 4.87 \\
& $\alpha_{\mathrm{H}}$ & 4.51 & 3.95 & 5.06 \\
& $\beta_{\mathrm{Fec}}$ & -0.25 & -0.62 & 0.12 \\
& $\beta_{\mathrm{SVL}}$ & -0.09 & -0.18 & 0.00 \\
& $\sigma_{\text {mass }}$ & 0.43 & 0.39 & 0.46 \\
& $\sigma_{\text {litter }}$ & 0.90 & 0.67 & 1.28 \\
& & & & \\
Neonate SVL & $\alpha_{\mathrm{R}}$ & 202.43 & 194.83 & 210.07 \\
& $\alpha_{\mathrm{H}}$ & 188.36 & 180.66 & 196.00 \\
& $\beta_{\mathrm{Fec}}$ & -4.19 & -10.11 & 1.92 \\
& $\beta_{\mathrm{SVL}}$ & 3.41 & -3.15 & 9.72 \\
& $\sigma_{\mathrm{SVL}}$ & 7.37 & 6.82 & 8.00 \\
& $\sigma_{\text {litter }}$ & 11.71 & 8.72 & 16.73 \\
\hline
\end{tabular}



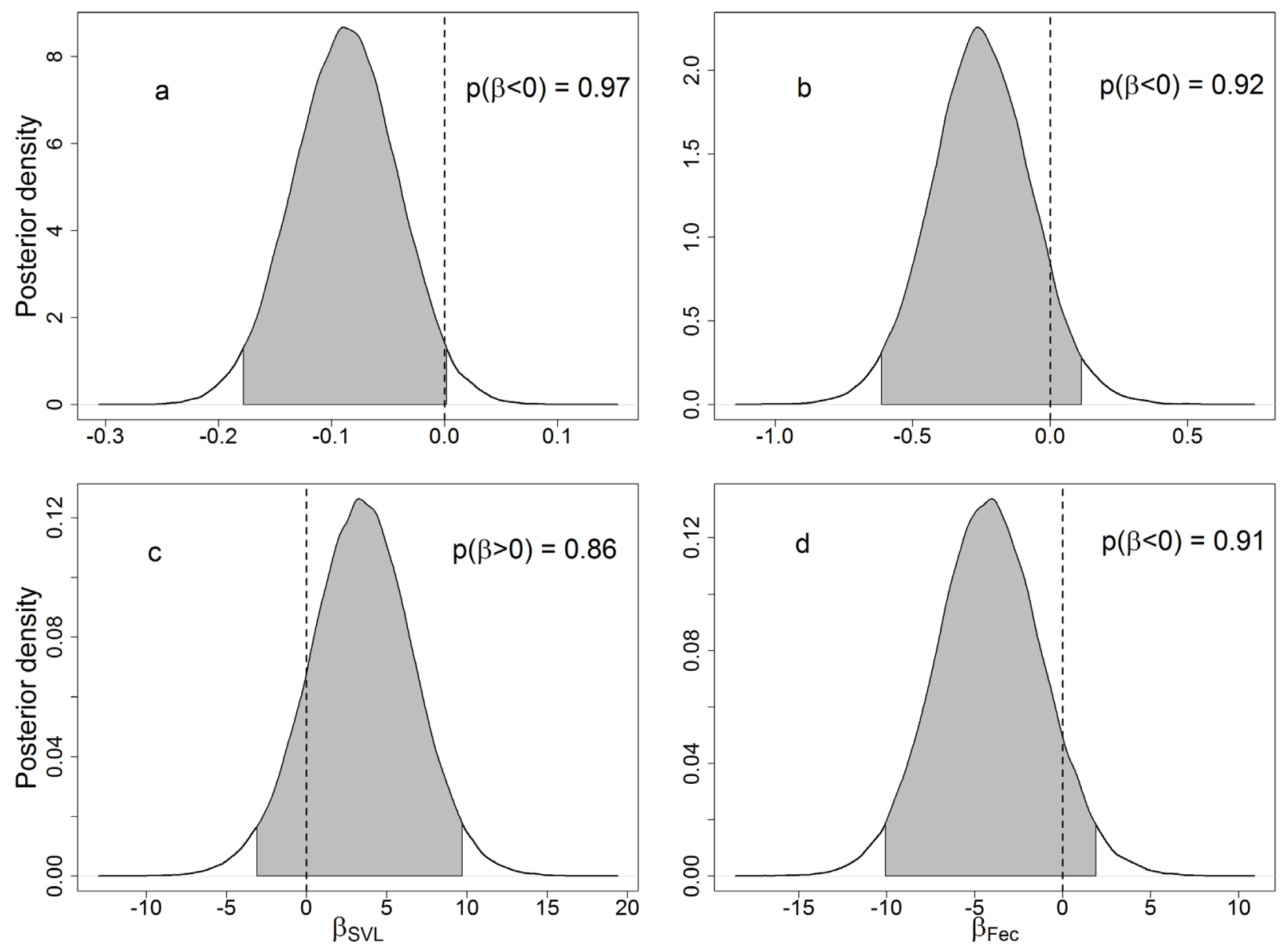

Figure 9. Posterior probability of slope parameters for the effect of maternal snout-vent length (SVL) of the giant gartersnake on (a) neonate mass or (c) neonate SVL, and for the effect of total litter size on (b) neonate mass or (d) neonate SVL. $\beta_{\text {Fec }}$ represents the effect of litter size on neonate size, and $\beta_{s v L}$ represents the effect of maternal SVL on neonate size. The vertical dashed line represents a value of 0 , the gray shading is the $95-$ percent credible interval of the parameter estimate, and the value in the upper right corner represents the posterior probability that the parameter has the same sign as its median estimate. 


\section{Annual Proportion of Gravid Females and Reproductive Frequency}

We performed $65 \mathrm{X}$-rays on 46 radio-telemetry snakes during the pre-parturition period; these snakes averaged $793.1 \mathrm{~mm}$ SVL (range $664-1018 \mathrm{~mm}$ ). The proportion of females that were gravid during the pre-parturition period was highest in 2016 (0.64; table 7), followed by 2014 (0.50) and 2015 (0.47). In 2014, the most snakes were examined in June, and in 2015 and 2016, the most snakes were examined in May (table 7). When we included year, Julian date (linear and quadratic), and maternal SVL as fixed effects in a binomial model of reproductive status, the estimated probability that a female was gravid was slightly higher in 2016 than $2015(0.16,-0.12-0.41 ; \mathrm{p}(2016>2015)=0.88)$ and 2014 $(0.25,-0.09-0.55 ; \mathrm{p}(2016>2014)=0.92)$, but there was no difference between 2014 and $2015(0.09$, $-0.20-0.36 ; \mathrm{p}(2015>2014)=0.73)$.

To get an estimate of the frequency with which individual females reproduce, we looked at a subset of 17 individuals that were examined with X-rays in 2 years or more during the pre-parturition period. Of 2 females examined in 3 years, 1 was gravid all 3 years, and 1 was gravid in only 1 of 3 years. Of the 15 females that were examined in 2 years, 4 were gravid in both years, 5 were gravid in 1 of 2 years, and 6 did not reproduce in either year. Overall, the average annual frequency of reproduction among 17 females examined in more than 1 year was 47 percent.

Table 7. Number of giant gartersnakes examined by X-ray and number gravid, by month, Sacramento Valley, California, 2014-2016.

[Only females examined from 1 May to 11 July are included]

\begin{tabular}{c|cc|ccccc|cc|c}
\hline \multirow{2}{*}{ Year } & \multicolumn{2}{|c|}{ May } & \multicolumn{2}{c|}{ June } & \multicolumn{2}{c|}{ July } & \multicolumn{2}{c|}{ Total } & \multirow{2}{*}{ Proportion } \\
\cline { 2 - 8 } & Examined & Gravid & Examined & Gravid & Examined & Gravid & Examined & Gravid & reproductive \\
\hline 2014 & 0 & 0 & 17 & 8 & 5 & 3 & 22 & 11 & 0.50 \\
2015 & 16 & 7 & 10 & 6 & 6 & 2 & 32 & 15 & 0.47 \\
2016 & 7 & 3 & 4 & 4 & 0 & 0 & 11 & 7 & 0.64 \\
\hline
\end{tabular}




\section{Discussion}

Despite its large size, many reproductive traits of giant gartersnakes appear to be similar to other Thamnophis and ecologically similar watersnakes in the genus Nerodia. The average litter sizes for giant gartersnakes estimated from X-rays (15.9) and captive litters (15.5) in this study are larger than that reported for T. marcianus (checkered gartersnake) (9.5) from Texas (Ford and Karges, 1987) and 6 populations of western terrestrial gartersnakes from California (5.2-10.5), but within the range of average litter sizes (7.6-23) reported for 11 populations of common gartersnakes distributed throughout Canada (Gregory and Larsen, 1993) and close to that reported for T. ordinoides (northwestern gartersnake) from Oregon (16.6) (Stewart and others, 1990). Female Nerodia sipedon sipedon (northern watersnake) in Ontario, Canada, and $N$. sipedon insularum (Lake Erie watersnake) in Lake Erie reach maximum lengths (asymptotic SVL $=920 \mathrm{~mm}, 1,015 \mathrm{~mm} \mathrm{SVL}$ ) similar to those of giant gartersnakes and produce larger litters on average (20 and 22.9, respectively) (King, 1986; Brown and Weatherhead, 1999a; Weatherhead and others, 1999; King and others, 2016). Larger female giant gartersnakes produce larger litters, and the slope of the maternal size-fecundity relationship from our linear regression model with untransformed maternal SVL (0.039) was similar to that reported for other new-world natricine snakes: Lake Erie watersnakes $(0.041)$, checkered gartersnakes (0.038), northwestern gartersnakes (0.06), common gartersnakes (0.054), and western terrestrial gartersnakes (0.035-0.047) (King, 1986; Ford and Karges, 1987; Stewart and others, 1990; Larsen and others, 1993; Bronikowski and Arnold, 1999, respectively).

In contrast to the clear maternal size-fecundity relation, our small sample of captive-born litters made it difficult to disentangle the effects of maternal size and litter size on neonate size. Our results indicate that neonates from larger litters were shorter and lighter than neonates from smaller litters. Surprisingly, larger females appeared to produce lighter neonates, after accounting for the effect of female size on fecundity. Studies of natricine snakes have generally found a positive relationship between maternal size and neonate size, after accounting for negative effects of litter size on neonate size (King, 1993; Weatherhead and others, 1999). Elucidating how maternal size interacts with litter size to affect neonate length and mass in T. gigas will require data on neonate size from a larger sample of litters.

The smallest gravid female recorded was $541 \mathrm{~mm} \mathrm{SVL}$, and 18 of 63 gravid females had SVLs less than $700 \mathrm{~mm}$. On the basis of growth trajectories of giant gartersnakes, it is likely that on average, females first reproduce at 3 years old (section 1). Female T. radix (plains gartersnake) and common gartersnakes have been reported to first reproduce at 2 or 3 years old (Fitch, 1965; Stanford and King, 2004; Tuttle and Gregory, 2012), and populations of western terrestrial gartersnakes vary in age at maturity from 3 to 5 years old (Bronikowski and Arnold, 1999). Therefore, the life history from our study populations appears to fit the general pattern seen in many Thamnophis species, and the large size of giant gartersnakes is not necessarily indicative of later maturity and higher fecundity than that of other gartersnakes. 
One area for further exploration is variation in reproductive traits throughout the range of giant gartersnakes. The average litter size of giant gartersnakes in this study (15.9 from X-rays and 15.5 from captive litters) was similar to the average of 17 neonates (range 13-21) reported by Halstead and others (2011b), but our litter sizes were smaller than the average litter size of 23 neonates (range 1046) reported by Hansen and Hansen (1990). The average size of neonates in this study (187.7 mm SVL) was smaller than that reported by Halstead and others (2011b; $208.8 \mathrm{~mm} \mathrm{SVL})$ and Hansen and Hansen (1990; $206.4 \mathrm{~mm} \mathrm{SVL})$. Halstead and others (2011b), collected females from four sites in the Sacramento Valley, including two sampled in this study (Central Colusa Basin, including Colusa NWR, and Sutter Basin, including Gilsizer Slough), but Hansen and Hansen (1990) measured captiveborn litters in populations in Sacramento County, the southern limit of our study area, and farther south, in the San Joaquin Valley. Unfortunately, because giant gartersnakes have been almost extirpated from the San Joaquin Valley (U.S. Fish and Wildlife Service, 2017), it is unlikely we will be able to collect reproductive data from southern populations for comparison. Likewise, little historical reproductive data are available for populations in the Sacramento Valley. Therefore, we are unable to evaluate whether the smaller neonate size and litter size found in this study represent a trend or natural spatial and temporal variability. Future studies could sample a variety of sites within the remaining distribution of giant gartersnakes to further the understanding of variation in reproductive traits. Because giant gartersnakes inhabit natural and restored wetlands, as well as agricultural canals, another potentially fruitful avenue for future work is to evaluate whether the reproduction and life history of this species varies among habitat types.

The data in this study were collected during a period of drought (2013-15) and the first average water year following a prolonged drought (2016). Some reproductive characteristics could be different during a period of normal or wet years. For example, a study in Kansas found that clutch or litter size was higher in years with higher rainfall for three snake species (Seigel and Fitch, 1985). Our results hint at potential annual variation in reproduction of giant gartersnakes because the proportion of females that were gravid was higher in 2016 than in 2014 and 2015. The proportion of females that reproduce each year is a key component of demographic models, and failure to accurately estimate this parameter could lead to overestimates or underestimates of productivity. Future studies during periods of normal or above-average rainfall would provide useful information on the natural variability in the reproductive ecology of this species.

A few issues related to our sampling methods may have affected the results of this study. First, most females we examined had radio transmitters surgically implanted. It is possible that carrying a radio transmitter affected the reproductive traits of these females, though this question has rarely been investigated for snakes. A study of Elaphe spiloides (gray ratsnakes) in Ontario, Canada, found that females with surgically implanted radio transmitters produced lighter clutches of eggs, relative to their body mass, than comparable females without radio transmitters (Weatherhead and Blouin-Demers, 2004). If the presence of radio-transmitters affected the ability of female giant gartersnakes to capture prey and therefore acquire energy stores to use in reproduction, it is possible that their frequency of reproduction and litter sizes would differ from females without radio transmitters. Future studies could collect reproductive data from a larger sample of females without radio transmitters to compare reproductive characteristics between females with and without radio transmitters. Also, because only large females could accommodate radio-transmitters, we did not X-ray small, but potentially sexually mature females. Thus our estimates of the proportion of adult females that are reproductive could be positively biased compared to the wider population. To obtain unbiased estimates of the proportion of reproductive females and to estimate the relationship between female size and the probability of reproduction, it would be beneficial to X-ray all captured females in the late spring and early summer to determine their reproductive status. 
This study shows the usefulness of X-radiography as a non-invasive method for counting litter size in snakes. Indeed, the fact that some non-reproductive females (confirmed from X-rays) were believed to be gravid, based on manual palpation, shows that palpation alone may not be reliable as a method of determining reproductive status. In the future, it would be valuable to compare embryo counts from X-rays to observed litter sizes in the same individuals. An advantage of X-radiography for measuring litter size is that it does not require females be kept in captivity for an extended period of time. One limitation of X-radiography is that it cannot be used to determine how many embryos are non-viable or how many neonates will be stillborn. Stillbirth might be a general phenomenon in giant gartersnakes; it has been documented in multiple populations and in studies separated by nearly 20 years (Halstead and others, 2011b). The proportion of live to stillborn neonates varied greatly among giant gartersnake litters, but on average, approximately 80 percent of neonates were born alive. Also, we found some evidence for a positive relationship between maternal size and the incidence of stillbirth in a litter, although we caution that our small sample limits our ability to elucidate this relation. The frequency of stillbirth in giant gartersnake litters could be a reason for concernincreased incidence of stillborn offspring was linked to inbreeding in an isolated population of Vipera berus (adder; Madsen and others, 1996). On the other hand, stillbirth has also been found in common, non-threatened natricine snakes such as western terrestrial gartersnakes, common gartersnakes, and northern watersnakes (Gregory and others, 1992; Weatherhead and others, 1998; Sparkman and others, 2007). For demographic studies of giant gartersnakes and other snakes in which stillbirth is common, estimates of fecundity based on X-rays need to be adjusted to account for the fact that not all embryos are likely to be born alive. More data on the frequency of stillbirth and variation in stillbirth among populations could help to elucidate drivers of this phenomenon and whether it represents a cause for concern in this threatened species.

This study is the first to document size-dependent fecundity and report reproductive frequency in female giant gartersnakes; it provides valuable information on reproduction in this threatened species. The relationship between fecundity and female size in giant gartersnakes and the indeterminate growth in this species (section 1) indicate that the largest, and therefore likely oldest females, have the greatest reproductive output. Results indicate that improved survival of large adult females should increase recruitment in giant gartersnakes, and thus it might be important to manage habitats with this in mind. Females spend greater than 50 percent of their time in terrestrial habitat, primarily taking refuge under cover, even during the active season (Halstead and others, 2015). Survival of large adult female giant gartersnakes is higher in terrestrial habitat than in aquatic habitat (Halstead and others, 2012). Ensuring that suitable terrestrial habitat is available near wetlands and canals, and limiting disturbance to terrestrial habitat when giant gartersnakes are likely to be present, could be important management actions to ensure that females can survive to older ages and produce large litters. 


\section{Section 3: Integrating Growth and Capture-Mark-Recapture Models to Estimate Size-Dependent Survival in Giant Gartersnakes}

\section{Introduction}

Wildlife populations are in decline worldwide. Under pressure from habitat loss, climate change, disease, introduced species, and overexploitation, distributions of native species have contracted, populations have decreased in abundance, and many are now faced with the threat of extinction (Brook and others, 2008; Dirzo and others, 2014). One way these drivers of species decline affect individual animals is to decrease their survival rate; thus, estimating the survival rate of individuals may be a useful metric for projecting future trends of population growth or decline. There is a wealth of literature devoted to estimating survival in animal populations on the basis of samples of marked individuals using capture-mark-recapture (CMR) methods (for example, Lebreton and others, 1992; Thomson and others, 2009). This work has been facilitated by the development of specialized software (White and Burnham, 1999) and the willingness of researchers to share their models with the wider community (Kéry and Schaub, 2012). As a result, we can now obtain answers to pressing conservation questions that would have been difficult to address in the past.

Even with these sophisticated tools, estimating survival of wild populations is difficult, especially for rare or elusive species that are infrequently observed or captured (hereafter, "elusive species"). Low capture probabilities lead to high uncertainty in estimates of survival (Pike and others, 2008; Papadatou and others, 2012). One solution to improve survival estimates when capture probabilities are low is to assume constant survival rates (Pollock and others, 1990). Survival often varies over time and among populations, however (Brown and Weatherhead, 1999b; Gaillard and Yoccoz, 2003; Miller and others, 2011), and it is important to quantify this variability to understand natural population dynamics (Rees and Ellner, 2009). Obtaining precise yearly or population-specific survival estimates is even more challenging for elusive species because increasing the number of parameters to be estimated from sparse data leads to greater uncertainty for each parameter (Pollock and others, 1990); this can inhibit the ability of researchers to estimate annual variation in survival (Hyslop and others, 2012). One way to improve precision while simultaneously obtaining site and year-specific survival estimates is to share information about the capture and survival process among years and among sites using a hierarchical model (Kéry and Schaub, 2012; Papadatou and others, 2012). For example, if the same species is sampled using the same methods at different sites, year after year, we may expect that the capture process should be very similar among sites and over time. Rather than treat data from each site and year as independent, we can allow the model to adaptively pool information on capture probabilities from each site and each year, and use these pooled data to increase the precision of estimates at each site in each year. 
In addition to modeling spatial and temporal variability in survival, there is a need to identify factors that drive variation in survival among individuals in the same population. Individual characteristics such as size, age, sex, and body condition can affect the probability of survival (Stanford and King, 2004; Altwegg and others, 2005; Blums and others, 2005). Including discrete variables such as age and sex as covariates of survival and capture probability is straightforward, provided an individual's state during its initial capture can be determined. If an individual is captured and classified once, its state is either constant (sex) or increases in a predictable, deterministic fashion (age). Measures of body size may be more closely related to survival than age, however (for example, in a population under a size-selective harvesting regime; Wallace and others, 2013), and some species are difficult to reliably age (Halliday and Verrell, 1988). Therefore a continuous measure of body size, such as mass or length, may be a better way to characterize an individual's condition.

Modeling the relationship between survival and continuous, time-varying individual covariates such as mass, length, or body condition is difficult because the state of the individual is unknown when it is not captured and measured. This problem is exacerbated for species with low capture probability because the vast majority of individuals may be captured only once or twice, and so most individual covariate data are missing. Recently, statisticians have developed methods to include time-varying individual covariates, such as body size, in CMR models using Bayesian imputation. In Bayesian imputation, a model is developed to predict the missing covariate values. These model-predicted covariate values, with their associated uncertainty, are used as covariates of the response variables of interest, such as capture probability and survival (Bonner and others, 2010; Schofield and Barker, 2011). Bayesian imputation can reveal previously obscured size-dependent relationships (Schofield and Barker, 2011, Rose and Todd, 2017) and is the preferred method for handling time-varying individual covariates when capture probabilities are low (Bonner and others, 2010). These advances empower us to answer questions about the relationship between body size and survival in elusive species that would have been difficult or impossible to address until recently.

Natricine snakes (family Colubridae, subfamily Natricinae) present good subjects for examining size-dependent survival because individuals rapidly increase in size early in life and survival often differs between juveniles and adults (Stanford and King, 2004; Miller and others, 2011). Body size may be a better predictor of survival rate than age because, although growth is usually rapid for young snakes and slows with age, adults can be highly variable in their maximum body size (King and others, 2016), and mortality resulting from predation may decrease for the largest individuals (Sparkman and others, 2013). Most studies of survival of natricine snakes have classified individuals into discrete classes on the basis of age or size and independently estimated survival for each class (Stanford and King, 2004; Lind and others, 2005). More recently, Hansen and others (2015) modeled size as a continuous covariate of survival in two populations of Thamnophis gigas (giant gartersnake). Hansen and others (2015) used a von Bertalanffy growth model to calculate the expected length of individuals when they were not captured and treated these expected size measurements as observed data in their CMR model. Hansen and others (2015) found that survival was positively related to snake size in the Natomas Basin, with large adult snakes having much higher survival than small, young snakes. The study by Hansen and others (2015) represents an important step forward, but using Bayesian imputation to incorporate uncertainty in snakes' sizes when they are not captured would better reflect variation in the growth trajectories among individual snakes and thus provide a more realistic model of this important covariate. In addition, Hansen and others (2015) included only a linear size effect on survival and did not test for more complex, non-linear relationships between individual size and survival probability. 
Survival is an important component of modern demographic models used for the conservation of threatened species, and the shape of the relationship between survival and individual size (for example, linear versus non-linear) affects conclusions about where to target management interventions (Easterling and others, 2000; Dahlgren and others, 2011). For example, some studies of natricine snakes have found survival initially increases with size to a peak, then declines for the largest or oldest individuals (Brown and Weatherhead, 1999b; Stanford and King, 2004), whereas others found support for a positive, linear relationship between size and survival (Hansen and others, 2015). If survival peaks for the intermediate sized individuals and declines for the largest individuals, this could have ramifications for the species' life history and for where managers should target conservation efforts. Modeling a more complex relationship between individual size and survival requires including additional terms in the survival model, such as a quadratic effect of size. We expanded on the methods used by Hansen and others (2015) to estimate survival and the size-survival relationship from 10 populations of the giant gartersnake in the Sacramento Valley of California. Specifically, we integrated a Bayesian von Bertalanffy growth model into a multi-state, hierarchical CMR model to fully account for the uncertainty in a snake's size when it is not captured and to share information on the capture and survival processes among sites and years. Our study had three main objectives.

1. Estimate the relationship between snake body size and survival.

2. Quantify variation in survival among populations and years.

3. Test for effects of precipitation, habitat composition, and prey abundance on survival.

The results have implications for the conservation and management of giant gartersnakes and their habitat, and illustrate the effectiveness of hierarchical Bayesian models for the study of rare and elusive species.

\section{Methods}

\section{Data Collection}

We performed CMR studies of giant gartersnakes at 10 sites within the Sacramento Valley, California, USA (fig. 10). Wetland habitat at our study sites comprised a mixture of remnant or created lentic wetlands and agricultural canals. The exceptions are Old Natomas North, Old Natomas Central, and Natomas 5, which comprised canals only, and Natomas 4, which comprised wetlands. Data were collected from 1999 to 2016, with individual sites sampled from 4 to 11 years (table 8). Only capture data from sites that were consistently sampled year after year for 4 or more years were used, although at some sites the position of the trap arrays changed between years to follow the availability of wetland habitat. We captured snakes using modified aquatic funnel traps (Casazza and others, 2000; Halstead and others, 2013) set in lines of 50-100 traps along the edges of canals or emergent vegetation in wetlands. At each site, 3-5 trap lines were set, resulting in a total of 150-250 traps per site. Sites were sampled from April to September each year, with an average of 72 consecutive days $(\mathrm{SD}=31)$ of sampling per site per year. We checked traps daily, processed snakes in the field, and released snakes on the day of capture, except for a small number of snakes that received radio transmitters as part of a separate study; these snakes were released approximately 7 days after capture. We sexed and measured the SVL of all snakes. Snakes were marked by branding ventral scales using a portable medical cautery unit (Winne and others, 2006), and PIT tags were inserted into snakes with greater than $30 \mathrm{~g}$ mass. 
Data on the habitat at each site were collected, including vegetation composition and prey abundance. We estimated the percent cover of emergent vegetation, floating vegetation, submerged vegetation, and terrestrial vegetation within a 1-meter $(\mathrm{m})$ radius of every fifth trap and within 10-20 circles with a 1-m radius randomly located along the trap array. Emergent vegetation at the study sites included Schoenoplectus acutus (tules), Typha spp. (cattails), and Ludwigia spp. (water primrose). Floating vegetation comprised Azolla spp. (mosquito fern), algae, and Lemna spp. (duckweed). Terrestrial vegetation was primarily grasses, weedy dicots, and small shrubs along the edge of wetland habitat. Submerged vegetation comprised Ceratophyllum demersum (coontail), Myriophyllum spp. (milfoil), and Potamogeton spp. (pondweed).

As an index of the prey base available at each site, the number of adult and larval anurans was counted, along with the number of fish within every fifth trap. The two anuran species present at the sites are the non-native Rana catesbeiana (American bullfrog) and the native Pseudacris regilla (Pacific treefrog), both of which are consumed by giant gartersnakes (Ersan, 2015). Many small fish that serve as prey for giant gartersnakes were captured by our minnow traps, including Gambusia affinis (western mosquitofish), Menidia beryllina (inland silverside), Lepomis spp. (sunfish), Micropterus spp. (black bass), and other non-native species in the families Cyprinidae, Centrarchidae, and Ictaluridae (Ersan, 2015).

Table 8. Summary of years and number of individual giant gartersnakes sampled at each site in Sacramento Valley, California, used in capture-mark-recapture analysis.

[NWR, National Wildlife Refuge]

\begin{tabular}{lllcc}
\hline \multicolumn{1}{c}{ Site } & Code & \multicolumn{1}{c}{ Years sampled } & Captures & Individuals \\
\hline Colusa NWR East & COE & $2012-2016$ & 152 & 78 \\
Colusa NWR West & COW & $2000-2005,2012-2016$ & 1478 & 675 \\
Gilsizer Slough & GI & $2007-2016$ & 676 & 377 \\
Natomas 1 & NAT1 & $2011-2016$ & 538 & 345 \\
Natomas 2 & NAT2 & $2011-2016$ & 263 & 166 \\
Natomas 3 & NAT3 & $2011-2016$ & 156 & 125 \\
Natomas 4 & NAT4 & $2011-2016$ & 13 & 9 \\
Natomas 5 & NAT5 & $2011-2016$ & 139 & 84 \\
Old Natomas North & NATN & $2000-2003$ & 135 & 90 \\
Old Natomas Central & NATC & $1999-2003$ & 220 & 136 \\
\hline
\end{tabular}




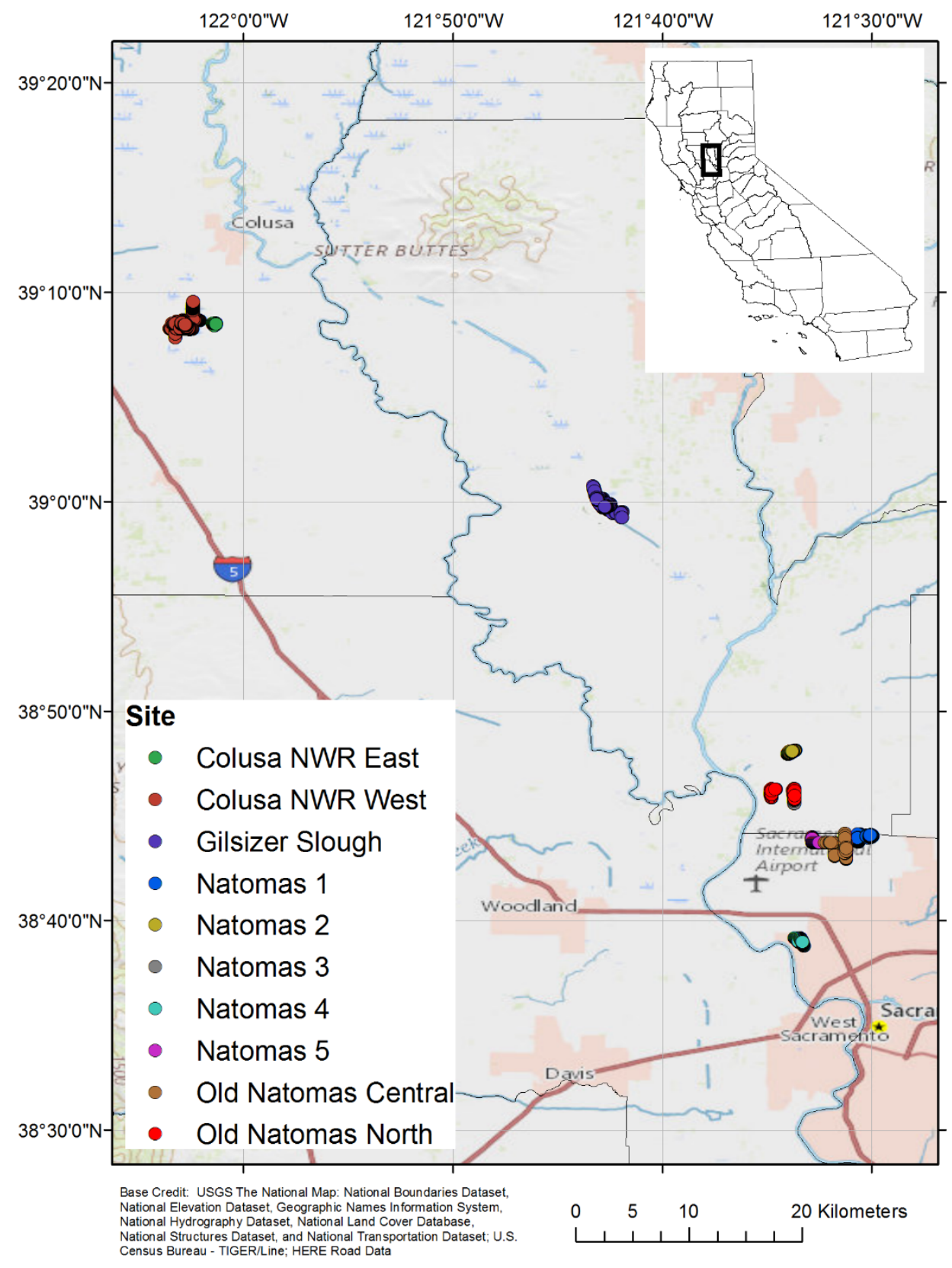

Figure 10. Location of capture-mark-recapture study sites within the Sacramento Valley, California. (NWR, National Wildlife Refuge) 


\section{Hierarchical Cormack-Jolly-Seber Model}

We fit a hierarchical, multi-state, robust-design Cormack-Jolly-Seber (CJS) model (Cormack, 1964; Jolly, 1965; Seber, 1965) to our CMR data to estimate annual apparent survival $(\phi)$, temporary emigration $\left(\gamma^{\prime \prime}\right)$, and daily (re)capture probability $(p)$ at each site in each year (Rankin and others, 2016). The model conditions on first capture, and individuals can occupy one of three states: dead, alive and outside the study area, and alive and inside the study area. Individuals are assumed to remain in the same state within a primary period (that is, population closure) but can change states between primary periods. Individuals temporarily emigrate from the study area with probability $\gamma^{\prime \prime}$ and stay inside with probability $1-\gamma^{\prime \prime}$. Individuals that are outside the study area remain outside with probability $\gamma^{\prime}$ and return to the study area with probability $1-\gamma^{\prime}$. We summed the number of captures for an individual in a given year and treated this total number of captures as a binomial response, with the size of the binomial equal to the number of days that a particular site was sampled that year.

Random effects of study site on $p, \phi$, and $\gamma^{\prime \prime}$, and nested random effects of year within site on all three parameters, were included. We modeled the probability of an individual staying offsite $\left(\gamma^{\prime}\right)$ as constant among sites and years because, with CMR data only, it is difficult for the model to estimate variation in this parameter.

Bayesian imputation was used to infer the size of snakes when they were not captured. We adapted the code from Schofield and Barker (2011) to implement a time-varying individual covariate in an open population model. We integrated a von Bertalanffy growth model for giant gartersnakes (section 1) into our hierarchical multi-state CJS model. The integrated growth model imputes a probability distribution for the size of a snake when it is not captured and therefore incorporates uncertainty in this unobserved covariate. We modeled linear $\left(\theta_{1}\right)$ and quadratic $\left(\theta_{2}\right)$ effects of snake SVL on $p$, as well as effects of sex (male, $\left.\theta_{3}\right)$ and trap modifications implemented in $2013\left(\bmod , \theta_{4}\right)$ on $p$, where $\omega$ is the intercept for capture probability on the logit scale (eq. 2 ).

$$
p=\frac{\exp \left(\omega+\theta_{1} * S V L+\theta_{2} * S V L^{2}+\theta_{3} * \text { male }+\theta_{4} * \bmod \right)}{1+\exp \left(\omega+\theta_{1} * S V L+\theta_{2} * S V L^{2}+\theta_{3} * \text { male }+\theta_{4} * \text { mod }\right)}
$$

We included linear $\left(\beta_{1}\right)$ and quadratic $\left(\beta_{2}\right)$ effects of snake SVL on $\phi$ (eq. 3). The logistic function asymptotes at a probability $=1$, but the average survival rate of large adult female $T$. gigas is much lower than $1(\hat{\phi}=0.61)$, based on radio-telemetry studies (Halstead and others, 2012). To allow for the size-survival function to asymptote at a probability of survival less than 1 , we included a "survival ceiling" parameter $(c)$ in the survival component of the CJS model (eq. 3).

$$
\phi=c * \frac{\exp \left(\alpha+\beta_{1} * S V L+\beta_{2} * S V L^{2}+\beta_{3} * \text { male }\right)}{1+\exp \left(\alpha+\beta_{1} * S V L+\beta_{2} * S V L^{2}+\beta_{3} * \text { male }\right)}
$$

The ceiling parameter is multiplied by the size-dependent survival probability and puts an upper limit on the survival rate (Hobbs and Hooten, 2015). For example, if survival exhibits a positive, linear relationship with size, and the ceiling parameter equals 0.8 , survival will increase with size and approach a maximum value of 0.8 , rather than 1 , for the largest individuals. We used an informative prior distribution for $c$, based on the mean estimated annual survival for radio-tracked adult female giant gartersnakes in the Sacramento Valley, with a slightly greater uncertainty around the mean (mean $=0.61, \mathrm{SD}=0.15$; Halstead and others, 2012). All other parameters had uninformative or weakly informative priors (table 9). 
Table 9. Cormack-Jolly-Seber model parameters, descriptions, and prior distributions.

[Normal distributions are defined by their mean and standard deviation, Half-Cauchy distributions by the location, scale, and degrees of freedom, Beta distributions by the two shape parameters ( $\alpha$ and $\beta$ ), and the Uniform distribution by lower and upper limits. SD, standard deviation]

\begin{tabular}{|c|c|c|c|c|}
\hline Model component & Parameter & Symbol & Prior distribution & Values \\
\hline \multirow[t]{7}{*}{ Survival } & Mean apparent survival & $\phi$ & Beta & $(1,1)$ \\
\hline & $\mathrm{SD}$, site random effect on $\phi$ & $\sigma_{\phi \text {-site }}$ & Half-Cauchy & $(0,1,1)$ \\
\hline & SD, year random effect on $\phi$ & $\sigma_{\phi-t}$ & Half-Cauchy & $(0,1,1)$ \\
\hline & Linear size effect on $\phi$ & $\beta_{\mathrm{SVL}}$ & Normal & $(0,1)$ \\
\hline & Quadratic size effect on $\phi$ & $\beta_{\mathrm{SVL} 2}$ & Normal & $(0,1)$ \\
\hline & Sex effect on $\phi$ (male) & $\beta_{\text {male }}$ & Normal & $(0,1)$ \\
\hline & Ceiling effect on $\phi$ & $c$ & Normal & $(0.61,0.15)$ \\
\hline \multirow[t]{7}{*}{ Capture } & Mean capture probability & $p$ & Uniform & $(0,1)$ \\
\hline & $\mathrm{SD}$, site random effect on $p$ & $\sigma_{p \text {-site }}$ & Half-Cauchy & $(0,1,1)$ \\
\hline & $\mathrm{SD}$, year random effect on $p$ & $\sigma_{p-\mathrm{t}}$ & Half-Cauchy & $(0,1,1)$ \\
\hline & Linear size effect on $p$ & $\theta_{\mathrm{SVL}}$ & Normal & $(0,1)$ \\
\hline & Quadratic size effect on $p$ & $\theta_{\mathrm{SVL} 2}$ & Normal & $(0,1)$ \\
\hline & Sex effect on $p$ (male) & $\theta_{\text {male }}$ & Normal & $(0,1)$ \\
\hline & Trap modification effect on $p$ & $\theta_{\text {mod }}$ & Normal & $(0,1)$ \\
\hline \multirow[t]{4}{*}{ Emigration } & Probability of staying offsite & $\gamma^{\prime}$ & Beta & $(1.3,1.3)$ \\
\hline & Probability of moving offsite & $\gamma^{\prime \prime}$ & Beta & $(1,1)$ \\
\hline & $\mathrm{SD}$, site random effect on $\gamma^{\prime \prime}$ & $\sigma_{\gamma^{\prime \prime} \text {-site }}$ & Half-Cauchy & $(0,1,1)$ \\
\hline & $\mathrm{SD}$, year random effect on $\gamma^{\prime \prime}$ & $\sigma_{\gamma^{\prime \prime}-t}$ & Half-Cauchy & $(0,1,1)$ \\
\hline Sex ratio & Proportion of males & $\operatorname{Pr}($ male $)$ & Uniform & $(0,1)$ \\
\hline \multicolumn{5}{|l|}{ Growth } \\
\hline & Asymptotic length (female) & $L_{\infty}$ & Normal & $(910.6,20.5)$ \\
\hline & Male effect on asymptotic length & $L_{\infty \text { male }}$ & Normal & $(-216.3,14.7)$ \\
\hline & Growth coefficient (female) & $k$ & Normal & $(1.308,0.241)$ \\
\hline & Male effect on $\mathrm{k}$ & $k_{\mathrm{m}}$ & Normal & $(0.296,0.143)$ \\
\hline
\end{tabular}

\section{Environmental Covariates}

To evaluate the importance of environmental variables, we fit a second CJS model to our CMR data that included annual precipitation, prey abundance (fish, frogs, and tadpoles), and habitat composition as covariates. This second CJS model was fit to our CMR data collected at Natomas 1-5, Colusa NWR West, Colusa NWR East, and Gilsizer Slough from 2011 to 2016 because those were the sites and years for which we had data on prey abundance and habitat composition. Annual precipitation for year $t$ is the amount of precipitation that fell during the water year from 1 October in year $\mathrm{t}-1$ to 30 September in year $\mathrm{t}$. Annual precipitation was calculated from the PRISM climatic database (PRISM Climate Group, 2016) and included precipitation in the previous year ( $\mathrm{t}-1)$ and precipitation in the current year $(\mathrm{t})$ of the survival interval as covariates in the model. We summarized prey abundance with three covariates representing the capture rate of fish, adult anurans (frogs), and larval anurans (tadpoles) at each site in each year. The capture rate of prey was calculated using a zeroinflated Poisson model implemented in Stan (Carpenter and others, 2017), which shares information 
among sites and years to provide better estimates for small samples (see section 1 for details of this model). Habitat composition covariates included the average percent cover of emergent, terrestrial, floating, and submerged vegetation at a site in a given year. All covariates (including snake SVL) were standardized before including them in the model in order to improve the efficiency of the Markovchain Monte Carlo (MCMC) sampler and make interpretation of model coefficients easier. Covariate values were standardized by subtracting the mean from each value and dividing by the standard deviation so that each standardized covariate had a mean of zero and standard deviation of one.

\section{Model Comparison and Model Averaging}

Indicator variable selection was used to estimate support for the inclusion of covariates and to compare alternative models (Kuo and Mallick, 1998). In short, each covariate was multiplied by a dummy indicator variable that can take on a value of 1 if the covariate is "turned on" and 0 if the covariate is "turned off." This binary indicator variable allows the MCMC sampler to choose whether or not a covariate effect is included in the model; important covariates are included in additional model iterations (Hooten and Hobbs, 2015). Each indicator variable had a Bernoulli(0.5) prior, so most covariates had a 50-percent prior probability of being included in the model. For quadratic effects of size on survival and $p$, we multiplied the indicator variable for the quadratic effect by the indicator variable for the linear effect so that quadratic effects could be turned on only if the linear effect was also turned on. The indicator variable for a quadratic size effect had a 25-percent prior probability of inclusion $(0.5 \times 0.5)$. The indicator variable for the survival ceiling parameter was designed such that if the ceiling effect was turned off, the parameter was set to 1 (for example, the usual logistic function), whereas if the ceiling effect was turned on, the $c$ parameter would take on a value of less than 1 chosen by the sampler. In the results, we present the posterior probability of individual models, and for covariates that appear important, we plot model-averaged relationships between the covariate and the response parameter ( $p$ or $\phi)$. We fit our CMR models in JAGS (version 4.2.0; Plummer, 2003), accessed through R (version 3.3.1; R Core Team, 2016) using the "runjags" package (Denwood, 2015). All models were run on 5 chains for 10,000 sampling iterations after an adaptation period of 5,000 iterations and a burn-in of 5,000 iterations. Mixing and convergence of chains were evaluated by inspecting trace plots and calculating the $\hat{R}$ statistic, which compares the within-chain variance to variances among chains (Brooks and Gelman, 1998). All parameters had $\hat{R}$ less than 1.05. Throughout the results, we present posterior means and symmetric 95-percent credible intervals for all parameters unless specified otherwise.

\section{Results}

\section{Capture Data Summary}

We made 3,770 captures of 2,085 individuals over the 17 years of study. The sex ratio of captured snakes from all sites was 1.2 females : 1 male; 1,123 individuals were female, 933 were male, and 29 were of unknown sex. The largest number of individuals was captured at Colusa NWR West, followed by Gilsizer Slough and Natomas 1 (table 8). Of 2,085 captured individuals, 1,838 were captured in only 1 year, 200 were captured in 2 years, 38 were captured in 3 years, and 9 were captured in 4 years. The longest interval between the first and last capture of a snake was 10 years, but most recaptured snakes were recaptured 1 year (63 percent of recaptures) or 2 years (25 percent) after their most recent capture. Within a year, individuals were captured from 1 to 30 times (mean \# of captures given a snake was captured once $=1.6, \mathrm{SD}=1.5$ ). 


\section{CJS Survival Model}

There was strong support for size-dependent capture probability, with a linear size effect included in 100 percent of model iterations and a quadratic effect in 99 percent of iterations (table 10). Capture probability exhibited a unimodal relationship with size, with the highest capture probability for individuals between 500 and $900 \mathrm{~mm} \mathrm{SVL}$. There was no support for a sex difference in capture probability. Capture probability was higher after trap modifications were implemented (fig. 11a). Even after accounting for the effect of trap modifications, there was large variation in capture probability attributable to site and year. The variation in daily capture probability among years $\left(\sigma_{p-\mathrm{t}}=0.0062,95\right.$ percent $\mathrm{CRI}=0.0036-0.0099)$ was much higher than variation among sites $\left(\sigma_{p \text {-site }}=0.0026,0.0001-\right.$ $\left.0.0071 ; \mathrm{p}\left(\sigma_{p \text {-t }}>\sigma_{p \text {-site }}\right)=0.96\right)$, but both represent substantial variation in this parameter. The SD of the year random effect on capture probability was 74 percent (56-96 percent) of the mean capture probability, whereas the standard deviation of the site random effect was 31 percent (1-78 percent) of the mean capture probability.

There was also clear support for size-dependent survival, with a snake's survival probability increasing with size, up to a peak, after which survival declines or plateaus (fig. 11b). The linear effect of size on survival was included in 100 percent of model iterations, and the survival ceiling parameter was included in 86 percent of iterations and all of the top eight ranked models. There was slightly weaker support for a quadratic effect of size on survival. The quadratic size parameter was included only in 64 percent of iterations, although its prior inclusion probability was only 25 percent compared to 50 percent for the linear size effect (table 10). The combination of these parameter effects on survival is illustrated by looking at the model-averaged relationship between snake size and survival. The model-averaged survival curve showed an initial increase in survival probability with size, going from a survival rate of less than 0.2 for snakes under $400 \mathrm{~mm}$ SVL to a peak survival rate of 0.5 for snakes 700-800 mm SVL, beyond which survival decreased slightly with increasing size on average (fig. 11b). There was substantial uncertainty around the size-survival relationship for very large snakes equal to or greater than $900 \mathrm{~mm}$ SVL. For the upper limit of the 95-percent credible interval, there is a plateau in survival for snakes equal to or greater than $700 \mathrm{~mm}$ SVL, but the lower limit of the 95percent credible interval exhibits a sharp decline in survival for snakes greater than $800 \mathrm{~mm}$ SVL. There was little support for sex differences in survival (table 10).

Table 10. The top eight models with posterior probability greater than or equal to 0.05 for the capture probability and survival of giant gartersnakes from 10 sites in the Sacramento Valley, California, sampled from 1999 to 2016.

[Each row represents a different model, the right-most column is the posterior probability of that model being chosen. The bottom row shows the probability of individual covariates being included in the model, based on the number of model iterations in which that covariate effect was included. Parameters are defined in table 9. $>$, greater than. Pr(Included) represents the posterior probability of that parameter being included in the model]

\begin{tabular}{|c|c|c|c|c|c|c|c|c|c|}
\hline Rank & $\theta_{\mathrm{SVL}}$ & $\theta_{\text {svL2 }}$ & $\theta_{\text {male }}$ & $\theta_{\text {mod }}$ & $\beta$ svL & $\beta$ svL2 & $\beta_{\text {male }}$ & c & $\begin{array}{l}\text { Posterior } \\
\text { probability }\end{array}$ \\
\hline 1 & 1 & 1 & 0 & 1 & 1 & 1 & 0 & 1 & 0.16 \\
\hline 2 & 1 & 1 & 0 & 1 & 1 & 1 & 1 & 1 & 0.14 \\
\hline 3 & 1 & 1 & 0 & 1 & 1 & 0 & 0 & 1 & 0.11 \\
\hline 4 & 1 & 1 & 0 & 1 & 1 & 0 & 1 & 1 & 0.08 \\
\hline 5 & 1 & 1 & 1 & 1 & 1 & 1 & 0 & 1 & 0.07 \\
\hline 6 & 1 & 1 & 0 & 0 & 1 & 0 & 0 & 1 & 0.06 \\
\hline 7 & 1 & 1 & 0 & 0 & 1 & 1 & 1 & 1 & 0.05 \\
\hline 8 & 1 & 1 & 0 & 0 & 1 & 1 & 0 & 1 & 0.05 \\
\hline $\operatorname{Pr}$ (Included) & $>0.99$ & 0.99 & 0.20 & 0.74 & $>0.99$ & 0.64 & 0.38 & 0.86 & \\
\hline
\end{tabular}



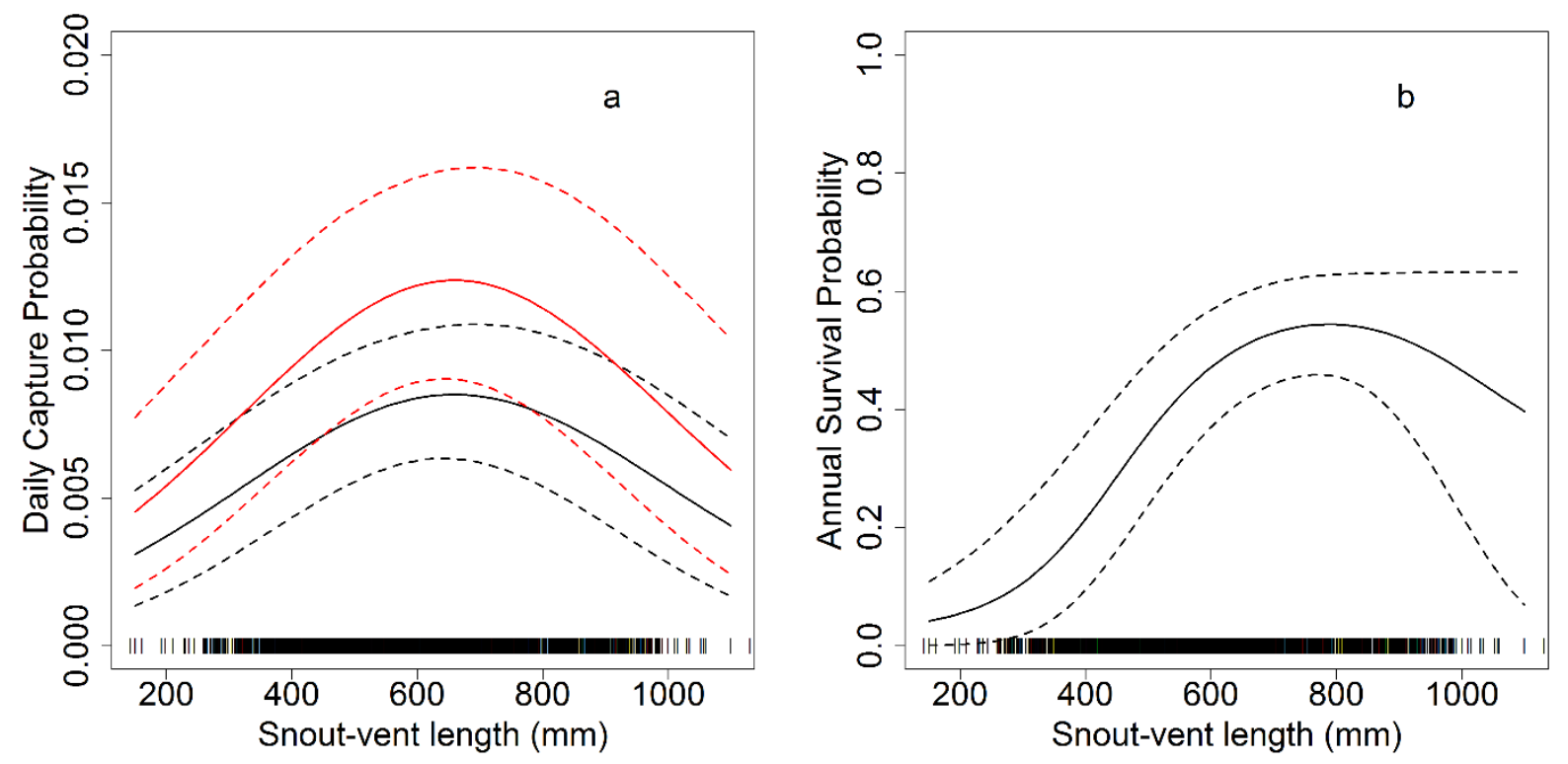

Figure 11. Model-averaged relationships between snake size and (a) daily capture probability (p) and (b) apparent annual survival ( $\phi)$. The red lines in panel a represent capture probability from 2013 to 2016 when traps were modified to improve capture rates. The black lines represent capture probability before these improvements. Solid lines represent the mean relationship for females; dashed lines are 95-percent credible intervals. Vertical dashes along the $x$-axis represent the sizes of captured snakes. (mm, millimeter)

The average survival probability, and the relationship between snake size and survival, varied among sites (fig. 12). For example, at Natomas 3, there is a greater contrast in survival between large and small snakes. However, at Natomas 1, survival for small snakes was relatively high compared to other sites, but the survival of large adults was lower than other sites (fig. 12). The average annual survival probability also varied over time and among sites (figs. 13-15). The average survival rate was highest at Colusa West $(\phi=0.58,0.47-0.71)$ and lowest at Natomas $1(0.39,0.18-0.59)$ and Natomas $5(0.39,0.15-0.60)$. The variation in survival among sites $(\mathrm{SD}=0.179,0.014-0.576)$ and years $(\mathrm{SD}=$ $0.177,0.016-0.394)$ was nearly equal, approximately 18 percent $(1.0-60.7$ percent) and 19 percent (1.3-54.8 percent) of the average annual survival, respectively.

Snakes had a nearly equal annual probability of remaining onsite or temporarily emigrating offsite, with the mean value of $\gamma^{\prime \prime}$ equal to 0.496 (table 11). The variation in temporary emigration among years $(\mathrm{SD}=0.168,0.01-0.344)$ was 36 percent $(2-88$ percent $)$ of the mean temporary emigration probability and was greater than variation among sites $(\mathrm{SD}=0.077,0.003-0.235)$, which was 17 percent (1-58 percent) of the mean value of $\gamma^{\prime \prime}$. The probability of a snake remaining offsite was greater than the probability of a snake immigrating back onsite $\left(\operatorname{Pr}\left(\gamma^{\prime}>0.5\right)=0.89\right)$. 
Table 11. Model-averaged parameter estimates and 95-percent credible intervals from the Cormack-Jolly-Seber model with all capture-mark-recapture data on giant gartersnakes collected from 10 sites in Sacramento Valley, California, from 1999 to 2016.

[Note the mean apparent survival, $\bar{\phi}$, must be multiplied by the survival ceiling parameter, $\mathrm{c}$, to get the predicted apparent survival value, $\phi$. SD, standard deviation; SVL, snout-vent length; \%, percent]

\begin{tabular}{|c|c|c|c|c|c|}
\hline \multirow{2}{*}{ Model component } & \multirow{2}{*}{ Parameter } & \multirow{2}{*}{ Symbol } & \multirow{2}{*}{ Mean } & \multicolumn{2}{|c|}{$95 \%$ credible interval } \\
\hline & & & & $2.50 \%$ & $97.50 \%$ \\
\hline \multirow[t]{7}{*}{ Survival } & Mean apparent survival & $\bar{\phi}$ & 0.715 & 0.431 & 0.964 \\
\hline & $\mathrm{SD}$, site random effect on $\phi$ & $\sigma_{\phi \text {-site }}$ & 0.179 & 0.014 & 0.576 \\
\hline & $\mathrm{SD}$, year random effect on $\phi$ & $\sigma_{\phi-\mathrm{t}}$ & 0.177 & 0.016 & 0.394 \\
\hline & Linear size effect on $\phi$ & $\beta_{\mathrm{SVL}}$ & 1.126 & 0.194 & 2.375 \\
\hline & Quadratic size effect on $\phi$ & $\beta_{\text {SVL2 }}$ & -0.278 & -0.886 & 0.005 \\
\hline & Sex effect on $\phi$ (male) & $\beta_{\text {male }}$ & -0.186 & -1.281 & 0.281 \\
\hline & Ceiling effect on $\phi$ & $c$ & 0.690 & 0.514 & 1.000 \\
\hline \multirow[t]{7}{*}{ Capture } & Mean capture probability & $p$ & 0.008 & 0.005 & 0.012 \\
\hline & $\mathrm{SD}$, site random effect on $p$ & $\sigma_{p \text {-site }}$ & 0.003 & 0.000 & 0.007 \\
\hline & $\mathrm{SD}$, year random effect on $p$ & $\sigma_{p-\mathrm{t}}$ & 0.006 & 0.004 & 0.010 \\
\hline & Linear size effect on $p$ & $\theta_{\mathrm{SVL}}$ & 0.063 & -0.231 & 0.335 \\
\hline & Quadratic size effect on $p$ & $\theta_{\mathrm{SVL} 2}$ & -0.120 & -0.196 & -0.037 \\
\hline & Sex effect on $p$ (male) & $\theta_{\text {male }}$ & -0.039 & -0.378 & 0.000 \\
\hline & Trap modification effect on $p$ & $\theta_{\bmod }$ & 0.375 & 0.000 & 0.892 \\
\hline \multirow[t]{4}{*}{ Emigration } & Probability of staying offsite & $\gamma^{\prime}$ & 0.649 & 0.411 & 0.863 \\
\hline & Probability of moving offsite & $\gamma^{\prime \prime}$ & 0.496 & 0.312 & 0.645 \\
\hline & $\mathrm{SD}$, site random effect on $\gamma^{\prime \prime}$ & $\sigma_{\gamma^{\prime \prime} \text {-site }}$ & 0.077 & 0.003 & 0.235 \\
\hline & $\mathrm{SD}$, year random effect on $\gamma^{\prime \prime}$ & $\sigma_{\gamma^{\prime \prime}-t}$ & 0.168 & 0.010 & 0.344 \\
\hline Sex ratio & Proportion of males & $\operatorname{Pr}($ male $)$ & 0.455 & 0.433 & 0.476 \\
\hline \multicolumn{6}{|l|}{ Growth } \\
\hline & Asymptotic SVL (female) & $L_{\infty}$ & 886.710 & 862.650 & 908.740 \\
\hline & Sex effect on asymptotic SVL (male) & $L_{\infty m}$ & -215.110 & -240.190 & -185.230 \\
\hline & Growth coefficient (female) & $k$ & 1.826 & 1.715 & 1.929 \\
\hline & Sex effect on growth coefficient (male) & $k_{\mathrm{m}}$ & 0.570 & 0.423 & 0.723 \\
\hline
\end{tabular}




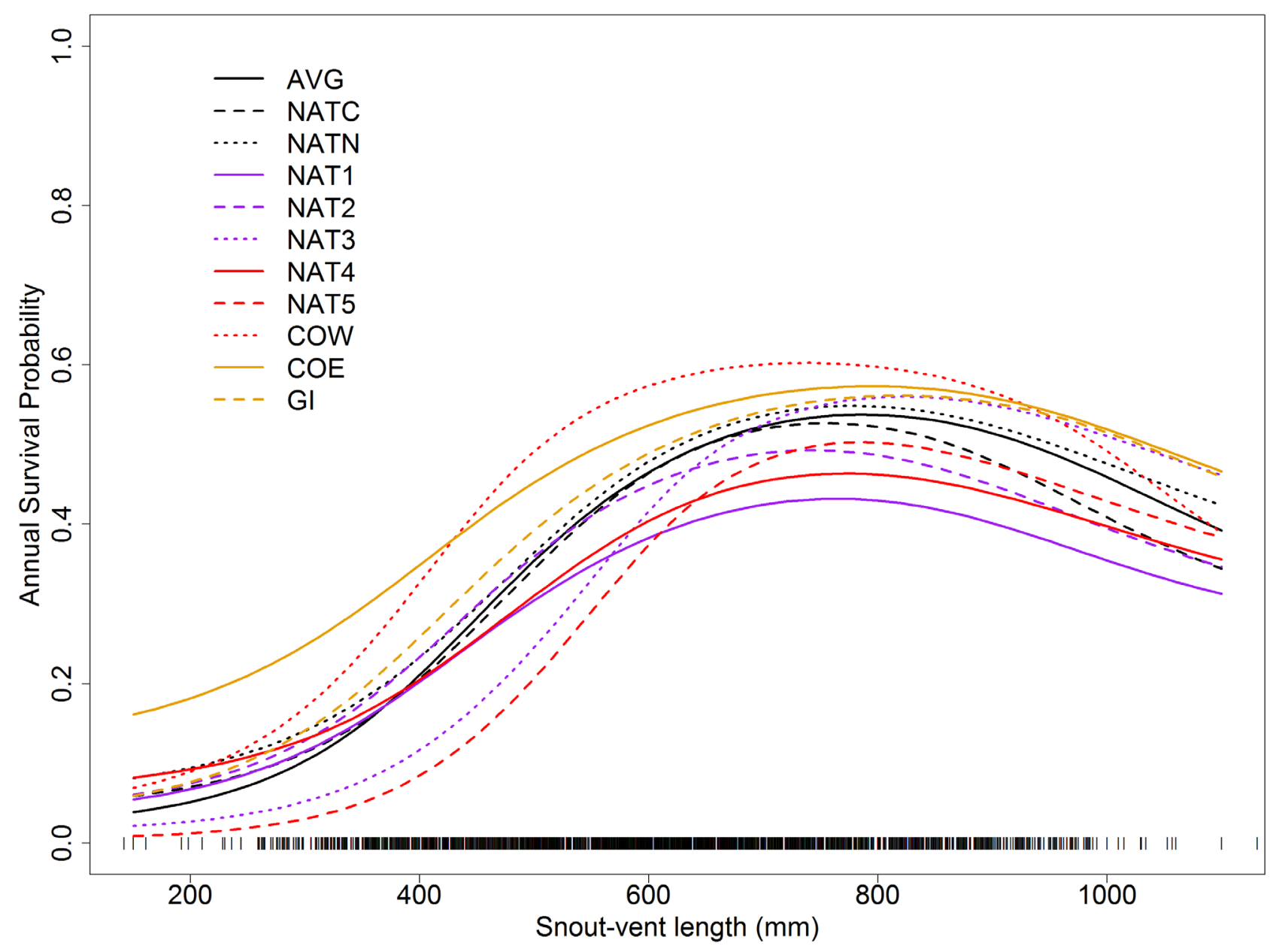

Figure 12. Mean size-survival relationships for each site in Sacramento Valley, California, along with the average of all sites. Vertical dashes along $x$-axis represent the snout-vent length of captured snakes. (Abbreviations for site names are defined in table 8; AVG, average) 


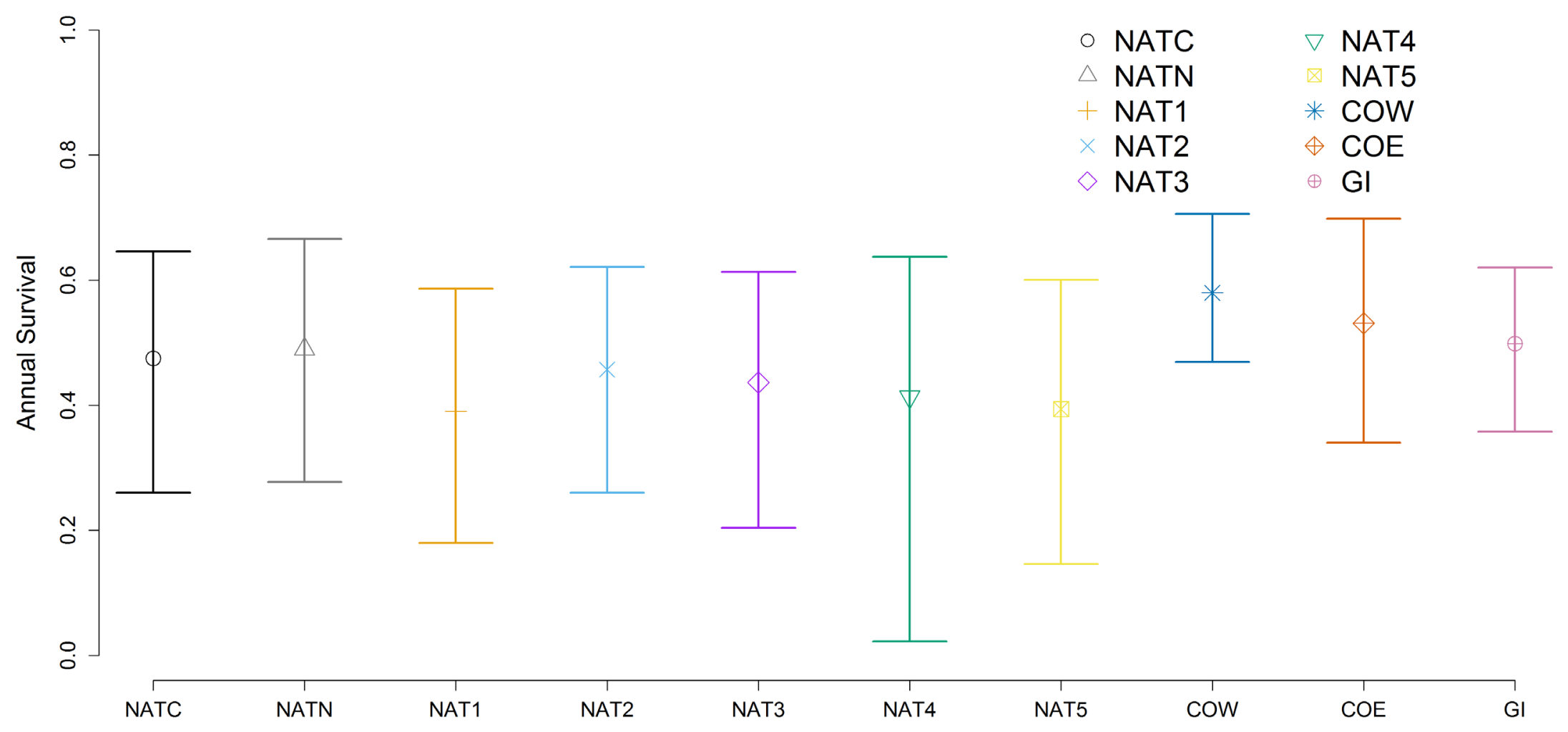

Figure 13. Average annual survival probability, $\phi$, for each site in Sacramento Valley, California. (Abbreviations are defined in table 8) 


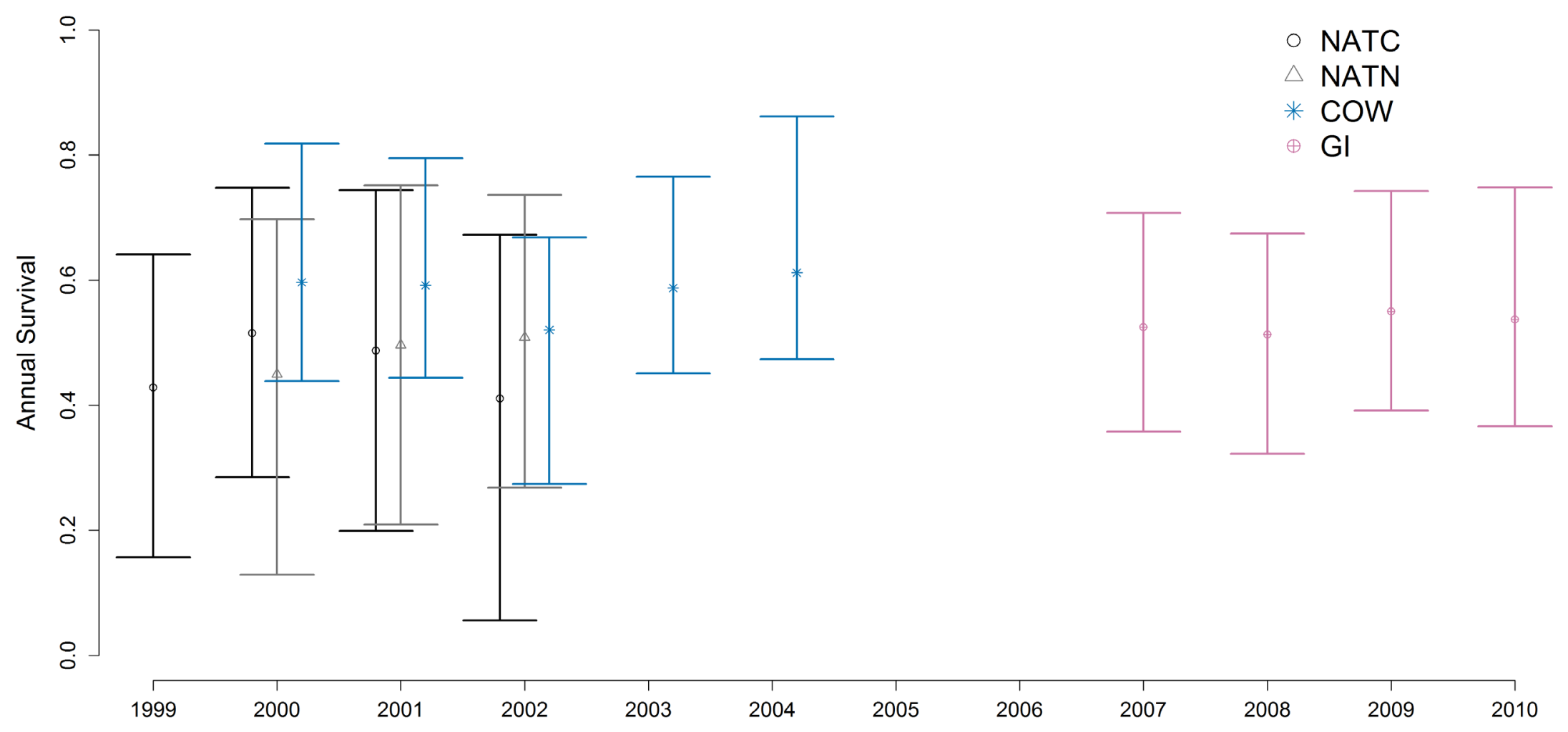

Figure 14. Average annual survival probability, $\phi$, by site in Sacramento Valley, California, for 1999-2010. (Abbreviations are defined in table 8) 


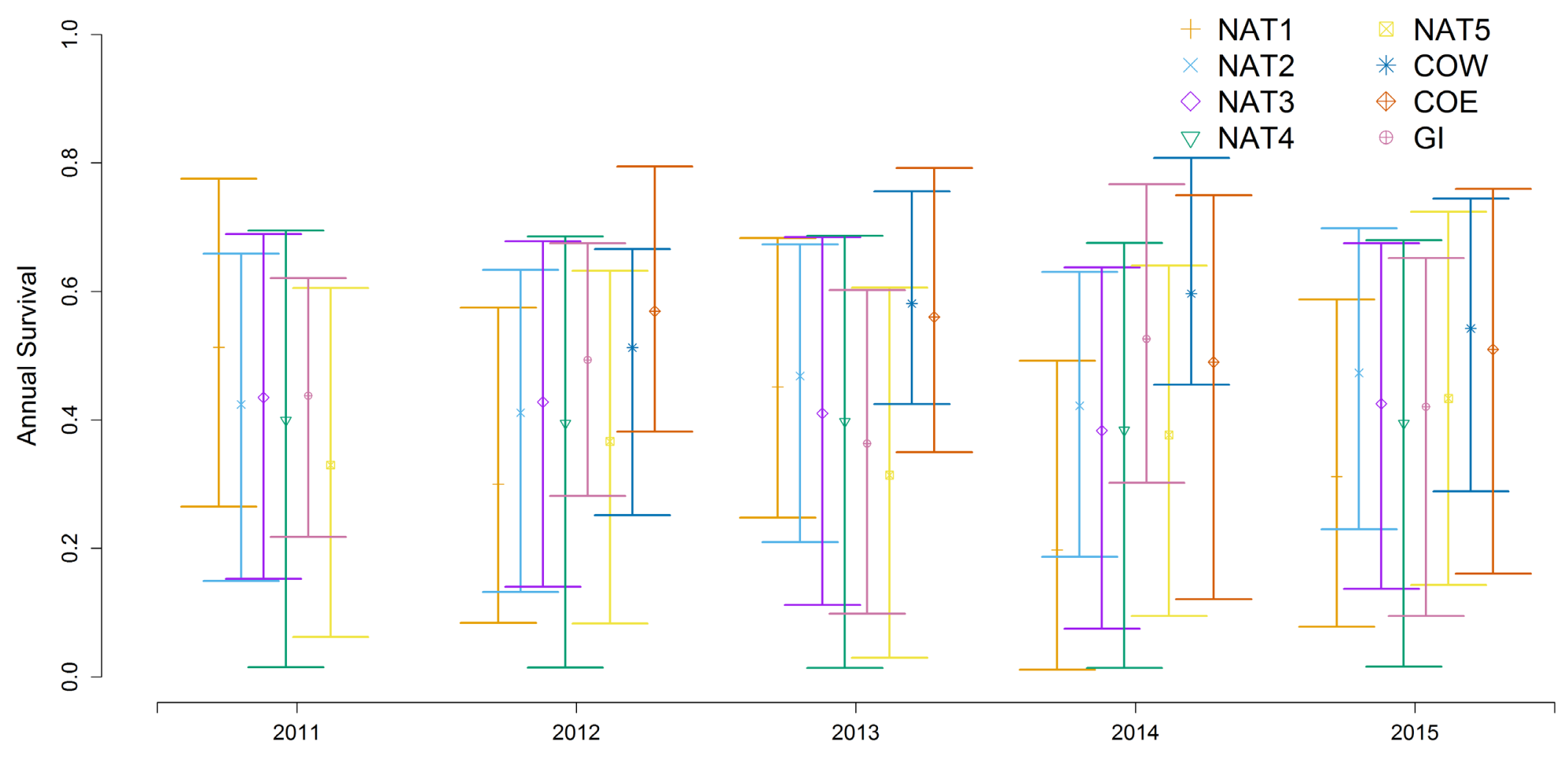

Figure 15. Average annual survival probability, $\phi$, by site in Sacramento Valley, California, 2011-15. (Abbreviations are defined in table 8) 


\section{Environmental Covariates of Survival}

For fitting the CJS model of environmental covariates of survival, we had 2,367 captures of 1,362 snakes at eight sites in the Sacramento Valley from 2011 to 2016. Emergent vegetation cover and fish abundance had the strongest relationship with survival, as these covariates were included in greater than 60 percent of model iterations and all of the top eight models. There was slightly weaker support for relationships between survival and previous-year precipitation and cover of floating vegetation, which were included in greater than 50 percent of model iterations (table 12). All other environmental covariates were included in less than or equal to 50 percent of model iterations. The top ranked model included effects of fish abundance, emergent vegetation cover, and floating vegetation cover on annual survival (table 12). The abundance of fish at a site and the proportion of emergent vegetation cover were both positively related to survival (table 13; fig. 16). Note that although the observed cover of emergent vegetation at a site ranged from 0 to 78 percent, most sites $(42 / 46)$ had less than 35 -percent emergent vegetation cover.

Table 12. The top eight models with posterior probability greater than or equal to 0.007 for the effect of environmental covariates on survival of giant gartersnakes from eight sites in the Sacramento Valley, California, sampled from 2011 to 2016.

[Each row represents a different model, the right-most column is the posterior probability of that model being chosen. The bottom row shows the probability of individual covariates being included in the model, based on the number of model iterations in which that covariate effect was included. Abbreviations are defined in table 13 . Pr(Included) represents the posterior probability of that parameter being included in the model]

\begin{tabular}{|c|c|c|c|c|c|c|c|c|c|c|}
\hline Rank & $\beta_{p p t, t-1}$ & $\beta_{\mathrm{ppt}, \mathrm{t}}$ & $\beta_{\text {fish }}$ & $\beta_{\text {frog }}$ & $\beta_{\text {tad }}$ & $\beta_{\mathrm{ev}}$ & $\beta_{\mathrm{tv}}$ & $\beta_{\mathrm{fv}}$ & $\beta_{\mathrm{sv}}$ & $\begin{array}{c}\text { Posterior } \\
\text { probability }\end{array}$ \\
\hline 1 & 0 & 0 & 1 & 0 & 0 & 1 & 0 & 1 & 0 & 0.010 \\
\hline 2 & 1 & 0 & 1 & 0 & 0 & 1 & 0 & 1 & 0 & 0.010 \\
\hline 3 & 1 & 1 & 1 & 0 & 0 & 1 & 0 & 1 & 0 & 0.009 \\
\hline 4 & 1 & 0 & 1 & 0 & 1 & 1 & 0 & 1 & 0 & 0.009 \\
\hline 5 & 1 & 0 & 1 & 1 & 0 & 1 & 0 & 1 & 0 & 0.009 \\
\hline 6 & 0 & 0 & 1 & 1 & 0 & 1 & 0 & 1 & 0 & 0.008 \\
\hline 7 & 1 & 0 & 1 & 0 & 0 & 1 & 0 & 1 & 1 & 0.007 \\
\hline 8 & 1 & 1 & 1 & 0 & 1 & 1 & 0 & 1 & 0 & 0.007 \\
\hline
\end{tabular}


Table 13. Model-averaged parameter estimates and 95-percent credible intervals for environmental covariates on survival from the Cormack-Jolly-Seber model using capture-mark-recapture data on giant gartersnakes collected in Sacramento Valley, California, 2011-2016.

[If a covariate was turned off in a given model iteration, its value was set to zero. \%, percent]

\begin{tabular}{llllll}
\hline \multirow{2}{*}{ Model component } & \multicolumn{1}{c}{ Parameter } & Symbol & Mean & \multicolumn{2}{c}{$\mathbf{9 5 \%}$ credible interval } \\
\cline { 5 - 6 } & & & & $\mathbf{2 . 5 0 \%}$ & $\mathbf{9 7 . 5 0 \%}$ \\
\hline Survival & Mean apparent survival & $\phi$ & 0.333 & 0.156 & 0.518 \\
& SD, site random effect on $\phi$ & $\sigma_{\phi-\text {-site }}$ & 0.154 & 0.004 & 0.565 \\
& SD, year random effect on $\phi$ & $\sigma_{\phi-\mathrm{t}}$ & 0.372 & 0.035 & 0.812 \\
& Precip (year t-1) effect on $\phi$ & $\beta_{\mathrm{ppt} \text { t-1 }}$ & 0.383 & -0.159 & 1.806 \\
& Precip (year t) effect on $\phi$ & $\beta_{\mathrm{ppt}, \mathrm{t}}$ & -0.004 & -1.044 & 1.080 \\
& Fish abundance effect on $\phi$ & $\beta_{\mathrm{fish}}$ & 0.394 & -0.054 & 1.412 \\
& Frog abundance effect on $\phi$ & $\beta_{\mathrm{frog}}$ & -0.110 & -1.307 & 0.770 \\
& Tadpole abundance effect on $\phi$ & $\beta_{\mathrm{tad}}$ & -0.233 & -1.456 & 0.405 \\
& Emergent vegetation effect on $\phi$ & $\beta_{\mathrm{ev}}$ & 0.630 & 0.000 & 1.918 \\
& Terrestrial vegetation effect on $\phi$ & $\beta_{\mathrm{tv}}$ & 0.117 & -0.241 & 1.021 \\
& Floating vegetation effect on $\phi$ & $\beta_{\mathrm{fv}}$ & 0.346 & -0.162 & 1.560 \\
& Submerged vegetation effect on $\phi$ & $\beta_{\mathrm{sv}}$ & -0.046 & -1.028 & 0.892 \\
\hline
\end{tabular}
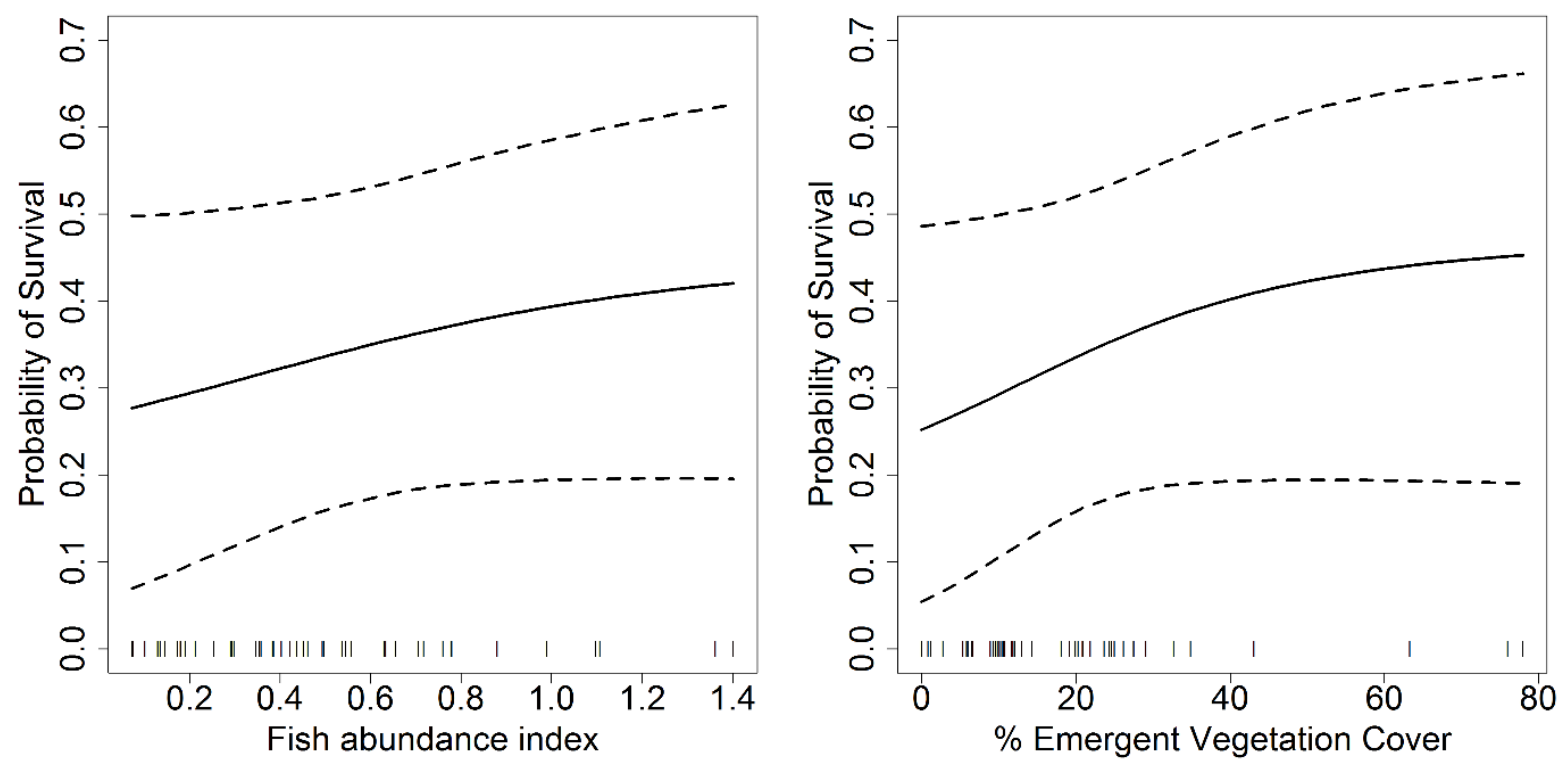

Figure 16. Model-averaged relationship between snake survival and (a) fish abundance and (b) percent cover of emergent vegetation, Sacramento Valley, California. Solid lines represent posterior mean relationships, and dashed lines are 95-percent credible intervals. Vertical dashes along the $x$-axis indicate observed values for the fish abundance and emergent vegetation covariates. 


\section{Discussion}

Integrating an individual growth model with a hierarchical, multi-state CMR model in a Bayesian framework enabled us to estimate the relationship between size and survival in a threatened species with very low capture probability. Survival was found to be positively related to individual size in giant gartersnakes. This relationship is not linear, however; survival only increases with size up to a point, after which survival either plateaus or decreases for the largest individuals. Even for large snakes with peak survival probability, the mean annual survival rate is only approximately 50 percent, which indicates this species is short-lived with high population turnover. Because a hierarchical model was used, we were able to obtain site and year specific estimates of survival and capture probability while estimating many fewer parameters than if we had treated site and year as fixed effects. Because of sparse capture data for some sites, we likely would have been unable to obtain year specific estimates of survival if we had analyzed data from each site separately, rather than in a hierarchical model. Survival varied substantially among both sites and among years within a given site. The observed spatial and temporal variation in survival highlights the need to incorporate such stochastic behavior in any demographic population model of giant gartersnakes.

The uncertainty in the size-survival relationship for large adult snakes is likely due to a combination of factors. First, we had few captures, and even fewer recaptures, of the largest individuals. Not only do large individuals make up a small proportion of the population, but only females reach lengths greater than $800 \mathrm{~mm}$ SVL, greatly limiting the number of individuals in this size range. Second, capture probabilities were low for the largest individuals as well, which reduces the precision with which we can estimate survival or any other vital rate of interest for large snakes. A final reason for the uncertainty in the survival of the largest snakes results from the model selection process. The uncertainty our model exhibits in choosing between a quadratic size-survival curve and a linear sizesurvival curve with a ceiling near 0.5 leads to a model-averaged curve that incorporates both of these possibilities. We think there is good reason to believe survival is relatively constant (that is, sizeindependent) for female snakes greater than approximately $800 \mathrm{~mm}$ SVL. Halstead and others (2012) used a hierarchical model to analyze survival data from several radio-telemetry studies of large adult female $T$. gigas, ranging from 648 to $1,040 \mathrm{~mm} \mathrm{SVL} \mathrm{(mean}=834 \mathrm{~mm}$ ). They found that the average annual survival rate of radio-tracked adult females was 0.61 , slightly higher than our estimate of approximately 0.5. Moreover, Halstead and others (2012) did not find support for an effect of snake SVL on survival within their sample of large adult females. Whether survival is nearly constant for giant gartersnakes above a certain size or declines slightly for the largest individuals, our results demonstrate that large adult snakes survive at a much higher rate than young, small individuals.

Our size-survival curves predict very low survival for small snakes, but we captured few snakes less than $300 \mathrm{~mm}$ SVL. The sparse capture data for very small snakes leads to great uncertainty in the survival of this size-class. These sizes represent individuals in their first year; by the time snakes are approximately 1 year old, they are greater than or equal to $400 \mathrm{~mm}$ SVL on average. Many more snakes in this 1-year-old class were captured than other age classes. Estimating the survival rate of neonate reptiles is notoriously challenging (Parker and Plummer, 1987; Pike and others, 2008). Some studies of natricine snakes have estimated the survival of neonates during their first year by releasing marked, captive-born neonates and recording the number recaptured or re-sighted the following year. A study of T. sirtalis fitchi (valley gartersnake) estimated first year survival probability to be 0.29 (Jayne and Bennett 1990), and a study of Nerodia sipedon sipedon (northern watersnake) estimated neonate survival probability was 0.31 from birth to emergence the following spring (Kissner and Weatherhead, 2005). Given the difficulty of capturing neonate giant gartersnakes, future demographic modeling 
efforts would be well served to model recruitment as the addition of 1-year-old snakes into the population because that is the age at which we can reliably begin tracking the growth, survival, and maturity of snakes. Nevertheless, designing studies to better quantify the first-year survival of giant gartersnakes might be important for understanding what drives variation in recruitment among sites and years. Specifically, it would be valuable to quantify the relative contributions of variation in female fecundity and variation in neonate survival to variation in recruitment over space and time.

The annual survival rates of giant gartersnakes in our study are similar to those from the only other comparable study of this species. Hansen and others (2015) studied two populations of giant gartersnakes over periods of 9 and 12 years. They estimated annual survival probabilities ranging from 0.27 to 0.62 in one population and found a positive relationship between snake size and survival in the other population. Although the Hansen and others (2015) study also documented size-dependent survival in giant gartersnakes, their estimates of survival for small snakes were much higher than ours. The smallest snake captured in the study by Hansen and others (2015) was $181 \mathrm{~mm}$ SVL and a snake this size was predicted to have an annual survival probability of 0.37 . Our model-averaged estimate of annual survival probability for a snake of the same size would be 0.03 . This discrepancy could be due in part to the difference in shape between linear and quadratic functions. We found support for a quadratic relationship between standardized snake SVL (scaled to have mean $=0$ and $\mathrm{SD}=1$ ) and survival. A unimodal quadratic function by definition exhibits a sharp decline on either side of the peak, and a positive logistic function asymptotes to zero for small values of a covariate. Because we had few captures of the smallest snakes, the low survival estimates for snakes with $400 \mathrm{~mm}$ SVL may be driven more by the shape of the quadratic curve than any biological process. Thus, our survival curves are best used to describe survival for snakes greater than 300-400 $\mathrm{mm} \mathrm{SVL}$, for which we have ample data.

When comparing our estimates of the annual survival rate of giant gartersnakes to those of closely related species, it is important to note that survival estimates vary among gartersnake species and among populations within a species. The annual survival probability was high in a population of $T$. atratus (aquatic gartersnake) in northern California, with females (0.64) having higher annual survival probabilities than males (0.56; Lind and others, 2005). A long-term study of $T$. radix (plains gartersnake) also found that the annual survival rate was sex and age dependent, with annual survival estimates comparable to our own. Mean annual survival probability estimates ranged from 0.16 for neonate snakes to a peak of 0.52 for 2-year-old females and 0.42 for 1- and 2-year-old males (Stanford and King, 2004). Survival, and the age dependence of survival, varies among ecotypes of the western terrestrial gartersnake, T. elegans, in northern California. The rate of annual adult survival in two populations with a fast life history was approximately 0.5 (but one such population had annual survival $\sim 0.8$ ), whereas in populations with a slow life history, the annual adult survival rate is usually greater than or equal to 0.7 (Miller and others, 2011). All three fast life-history populations of western terrestrial gartersnakes exhibited an increase in survival with age, whereas only 1 of 3 slow life-history populations had a positive relationship between age and survival (Miller and others, 2011). Annual survival was very high in a population of the endangered $T$. sirtalis tetrataenia (San Francisco gartersnake; $\geq 0.75$ in each of 2 years) (Halstead and others, 2011a). Annual survival of the semiaquatic northern watersnake in two populations in Ontario, Canada, (0.53-0.58) is similar to our estimates for giant gartersnakes (Brown and Weatherhead, 1999b). Annual survival from two largebodied, long-lived, terrestrial Colubridae species in North America is slightly higher than our estimates for T. gigas. The CMR estimate of annual survival rate for Elaphe spiloides (gray ratsnake), in Ontario, Canada, was 0.68 (Row and others, 2007), and the annual survival rate of the threatened snake Drymarchon couperi (eastern indigo snake) in Georgia, USA, was estimated at 0.7 (Hyslop and others, 2012). Survival is generally much higher in the family Viperidae than in natricine snakes. Adult annual survival was greater than 0.7 for six populations of Vipera aspis (asp viper) in Switzerland, although 
survival of juveniles was less than 0.5 in 4 out of 6 populations (Altwegg and others, 2005). In a longterm study of Crotalus horridus (timber rattlesnakes) in northeastern New York, annual survival of subadults and adults was greater than 0.8 , and survival of neonates ranged from 0.53 to 0.77 , depending on their color morph (Brown and others, 2007). Although an exhaustive review of age and size-dependent survival patterns of snakes is beyond the scope of this study, it is likely that despite their larger size, the annual survival rate of giant gartersnakes is much closer to that of other gartersnakes and new-world natricines than long-lived terrestrial Colubridae and several species of Viperidae.

Modeling a continuous size-survival relationship has several advantages over binning individuals into discrete "size classes." First, any classification based on size ranges will be somewhat arbitrary, but these decisions about the width and number of size classes in a demographic model can affect conclusions about what drives population growth (Enright and others, 1995). Second, the more size classes there are, the less precise estimates of survival for each class will be. Increasing the number of size classes decreases the sample size in each class, which limits the data available to estimate a mean and variance for each size class. In contrast, modeling a continuous relationship between size and survival requires only estimation of 3 or 4 parameters, survival intercept, slopes for the size effects on survival (linear, and quadratic if necessary), and variance around the mean relation. Also, once you have fit a continuous size-survival function, it is trivial to discretize that continuous function and define size classes. Going the other direction and translating from discrete, size-class-based estimates of survival to a continuous size-survival function is problematic.

We modeled variation among sites in the capture probability of giant gartersnakes using random effects, which assumes that the capture process is similar among sites, and differences among sites can be represented as values drawn from a shared normal probability distribution. This allowed us to "borrow strength" from sites with many recaptures to improve the precision of estimates of capture probability at sites with few captures. It is possible that the capture process could differ among sites in more systematic ways, however. For example, if traps can sit flush to the wetland/canal bank at some sites, such that the bank acts as a drift fence to guide snakes into traps, capture probabilities may be higher than at other sites where the bank does not act as an effective drift fence. If our assumption about the exchangeability of the capture process among sites were violated, it could potentially affect estimates of survival. For example, imagine three sites: sites A and B have many recaptures of marked individuals, and site $\mathrm{C}$ has very few. Thus, sites $\mathrm{A}$ and $\mathrm{B}$ contribute much information to our model of the capture process, whereas site $\mathrm{C}$ contributes little information, and its estimated capture probability is shrunk towards the overall mean for all sites. If the true probability of recapturing marked snakes at site $\mathrm{C}$ were much higher than other sites, but survival was low, this would also result in few actual recaptures. Then, our estimate of $p$ for site $\mathrm{C}$ would be an underestimate, and we might overestimate $\phi$ at site $\mathrm{C}$. We do not think this type of systematic variation in capture probability among sites is a major factor for two reasons. First, there was more variation in capture probability among years than among sites, indicating that annual variation is more important. Second, we use the same trapping methods at each site to ensure uniformity as much as possible. Still, we cannot exclude the possibility that unmodeled heterogeneity in the capture process among sites affected our results. It could be beneficial in the future to explicitly test for environmental factors that explain some of the heterogeneity in capture probability among sites and among years, we found in this study. 
Another factor that complicates estimating survival from wild populations is the movement of individuals off the sample array. Temporary emigration of individuals from the sampled area leads to negatively biased estimates of apparent survival, unless it is explicitly included in a CMR model (Kendall and others, 1997). Temporary emigration was accounted for by using a multi-state model, in which individuals that were not recaptured could take on 1 of 3 states: (1) alive, onsite, but uncaptured; (2) alive and offsite; and (3) dead. Still, even though we modeled the temporary emigration process, it is possible we underestimated true survival. First, because the capture probability of giant gartersnakes is low, few data are available to differentiate between an individual that temporarily moves offsite and an individual that remains onsite but goes uncaptured in 1 year or more. Estimates of the temporary emigration parameter could potentially be improved, and thus our apparent survival estimates, by using auxiliary data on the movement of giant gartersnakes from radio-telemetry studies (for example, Powell and others, 2000). Second, our apparent survival parameter, $\phi$, confounds the true survival rate, $S$, and the probability an individual permanently emigrates from the site. One way to address this in the future would be to use a spatial capture-recapture model, which estimates the movement of individuals on and off of the sampling array on the basis of the exact location where an individual is captured (Ergon and Gardner, 2014). Although these new spatial methods are promising, they may be difficult to implement for giant gartersnakes because spatial capture-recapture requires repeated, spatially distinct captures of individuals within a year to estimate their activity center and movement pattern (Royle and others, 2014). Given the low capture probabilities for individual giant gartersnakes, it remains to be seen whether our data contain enough spatially distinct recaptures of the same individuals to use a spatial capture-recapture model.

In addition to the relationship between survival and snake size, we found survival of giant gartersnakes was related to the cover of emergent vegetation and the abundance of fish at a site. Radiotelemetry studies show that giant gartersnakes select areas with higher cover of emergent vegetation, and occupancy studies show that snakes primarily are present in or near areas that were historically tule marsh (Halstead and others, 2014, 2016). Thus, it is intuitive that snakes survive at a higher rate in habitats that have more emergent vegetation cover, which better replicates the tule marshes these snakes inhabited before European-American settlement. We caution that the positive relationship between emergent vegetation cover and snake survival may not extend to sites with greater than 50 percent emergent vegetation cover. Giant gartersnakes likely need a mix of emergent vegetation and open water in which to forage, and wetland habitats that are choked with thick emergent vegetation are likely suboptimal for giant gartersnakes.

Growth in giant gartersnakes is related to fish and frog abundance (section 1), but the relationship between growth and fish abundance is only positive when frog abundance is very low. Giant gartersnakes prefer anuran prey over fish, but fish make up a large percentage (about 40 percent) of their diet in the wild (Ersan, 2015). It is curious that fish would be positively related to the survival of giant gartersnakes while their preferred prey, anurans, are not. Given that most of the sites inhabited by giant gartersnakes are novel habitats created and managed by humans (for example, agricultural canals, created wetlands), the makeup of their ecological communities is affected by the manner in which people manage water over time. The abundance of fish might be related to the survival of giant gartersnakes, not only because it represents how much food is available, but also because the abundance of fish is an indicator of overall habitat quality and of the permanence of aquatic habitat. Giant gartersnakes need aquatic habitat throughout their active season from spring through the early fall. Fish will only persist and be abundant in more permanent water bodies; otherwise, they must continually recolonize wetland habitats once they are flooded in the winter or spring. 


\section{Conclusions}

Our study demonstrates the power and flexibility of Bayesian hierarchical models for modeling the survival of elusive species and the effect of individual and environmental covariates on survival and detection. Survival increases with size in giant gartersnakes, but once individuals reach adult size, their survival rate plateaus or decreases slightly with further growth. This life-history pattern has potential ramifications for the conservation and management of this threatened species. The relatively low annual survival rate of larger adults indicates that few females will survive to reach very large body sizes and therefore become highly fecund (section 2). We also found that the survival of giant gartersnakes was positively related to the abundance of fish and the cover of emergent vegetation within wetlands, which provides managers valuable information on how to improve managed wetlands for this species. The results provide much needed information on the demography of this threatened species and further the understanding of the life history of species in the genus Thamnophis. Our findings indicate that hierarchical models that share information among sites and years could be widely applied to the study of rare and elusive species to improve researchers' ability to extract the most information from available data. 


\section{Section 4: Development and Elasticity Analysis of an Integral Projection Model for the Giant Gartersnake}

\section{Introduction}

An increasing number of threatened and endangered species, growing threats from myriad stressors, and limited resources make it imperative to maximize the effect of conservation and management actions on high priority species. Demographic population models are one of the primary tools used by researchers, managers, and conservationists to evaluate the effectiveness of potential conservation interventions (Morris and Doak, 2002). Demographic models integrate data on vital rates such as the survival, growth, and reproductive output of individuals to model the state and simulate the dynamics of the population as a whole. Demographic models are especially useful in conservation because they can be used to determine which vital rates will have the greatest effect on the trajectory of the population in the future (for example, calculating sensitivities or elasticities) and therefore provide clear targets for conservation actions (Caswell, 2001). Because demographic models have proven so valuable for ecology, conservation, and management, new and improved methods are developed at a rapid pace.

Matrix population projection models are perhaps the most commonly used demographic models in studies of wildlife populations (Leslie, 1945; Caswell, 2001). Matrix models are popular because the math involved is intuitive and the discrete time structure of these models (that is, regular, evenly spaced time steps) matches well with the regularly spaced sampling designs used to study wildlife populations (for example, yearly or monthly surveys for marked individuals of a focal species). Although these models are powerful tools, they require either information on the age of all individuals in the population or the classification of individuals into discrete life-history stages or size classes. Reptiles are notoriously difficult to age, and estimates of age based on age-size relationships have great uncertainty associated with them. Because the growth rate of reptiles declines with age (Andrews, 1982), and because individuals vary in their adult size (King and others, 2016), it is very difficult to assign an age to a very large, adult individual, unless it was initially captured shortly after birth. Generally, the most researchers can say about the age of a large adult captured for the first time is that it must be greater than $\mathrm{X}$ years old, where $\mathrm{X}$ is the age at which individuals usually reach sexual maturity. Some researchers have attempted to estimate the age of reptiles on the basis of growth layers in bones (skeletochronology), but this method is subject to observer error and requires cutting off the tip of the tail to obtain a cross section of vertebrae (Waye and Gregory, 1998). This type of destructive sampling is undesirable when studying a listed species.

Size- or stage-based matrix models are used for species that are difficult to age or that have discrete life-history stages (for example, frogs with egg, tadpole, metamorph, and adult life stages). Researchers classify individuals into discrete size classes or stages, then estimate vital rates (survival, growth, fecundity) for each of those stages and use these values to create a demographic model (Lefkovitch, 1965; Caswell, 2001). Although this method is widely used and is an intuitive adaptation of the age-based matrix model for species for which aging is difficult, it has a few shortcomings. First, the divisions of individuals into size classes is arbitrary for species without distinct life-history stages. For example, it is not clear what would be appropriate size classes for modeling the demography of a rapidly growing snake, such as Thamnophis gigas (giant gartersnake), because its biology does not neatly map into a few life-history stages. Second, the manner in which individuals are classified into size classes affects the results of the demographic analysis, even if the underlying data are the same (Enright and others, 1995). For example, although creating a matrix with fewer size classes may allow for more precise estimates of vital rates (because of the larger sample size for each size class), it means 
that vital rates are averaged over a larger range of individual sizes, which may obscure important variation among individuals (Moloney, 1986). Creating a matrix with many size classes may better represent continuous variation in vital rates with changes in individual size, but building a matrix with many size classes means each size class has a smaller sample size and thus less precise parameter estimates (Moloney, 1986). Furthermore, having a small number of size classes in the model generally increases the apparent importance of survival and stasis within the same size class for population growth, based on elasticity. This happens because with few size classes, the range of sizes within a class is large, and the probability of an individual surviving and growing into the next size class is lower than the probability of surviving and remaining in the same size class (Enright and others, 1995). In contrast, a model with many size-classes increases the apparent importance of growth into the next size class because the probability of an individual surviving and staying in the same size class will, in general, be small relative to the probability of surviving and growing into the next size class (Enright and others, 1995). Finally, the ultimate goal of many population modeling efforts for threatened and endangered species is to apply a stochastic model to project population viability (Fieberg and Ellner, 2001; Morris and Doak, 2002). Modeling environmental stochasticity using a matrix model requires estimating the mean and variance for each component of the matrix, along with the covariance parameters among all matrix components (Rees and Ellner, 2009). Simultaneously estimating so many parameters may not be possible with sparse datasets for rare or elusive species for which capture probability is low.

To avoid some of the shortcomings of matrix population models, Easterling and others (2000) developed the IPM. Similar to a matrix model, an IPM is a discrete-time model that iterates on regular, evenly spaced time steps. Unlike a matrix model, the state variable in an IPM can be a continuous character, which allows researchers to better model the survival, growth, and fecundity of individuals on the basis of continuous traits, such as size. There are many advantages to using an IPM to model the demography of the threatened giant gartersnake. First and foremost, there is extensive evidence that survival (Brown and Weatherhead, 1999b; Hansen and others, 2015; section 3), growth (Coates and others, 2009; section 1), and fecundity (Weatherhead and others, 1999; Stanford and King, 2004; section 2) vary with size in the snake sub-family Natricinae, and in giant gartersnakes specifically. These sizevital rate relationships are usually estimated with regression models (for example, generalized linear models), which are simple to translate into the functions needed to construct an IPM. In reptiles, somatic growth rates (King and others, 2006), annual survival (Brown and Weatherhead, 1999b), and fecundity (Gregory and Larsen, 1993) may vary among populations and among years. IPMs naturally accommodate spatial and temporal variability by using a mixed-effects approach to estimating growth, fecundity, and survival of animals in different populations and among different years (Rees and Ellner, 2009). This enables us to integrate data from different sites and different years, and simultaneously estimate the mean size-vital rate relationship, along with the specific relationships for different populations and different years. Data on temporal variation in vital rates can be used to construct stochastic population models that reflect natural variability in fecundity, somatic growth, and survival. Stochastic population models then can be used in Population Viability Analyses (PVAs) to evaluate the relative probability of a threatened population or species going extinct under different management or conservation scenarios (Fieberg and Ellner, 2001). 
The goal of this analysis is to develop a demographic population model for giant gartersnakes. Specifically, our goal is to evaluate if and how three key vital rates - survival, growth, and fecundityare related to snake size and how important each of these vital rates is to the population growth of giant gartersnakes. Within this overall goal are four component objectives. First, we aimed to estimate size or life-stage specific demographic rates from existing USGS project data on giant gartersnake populations, data from other researchers who study giant gartersnakes, or from the scientific literature. These demographic rates include the annual survival rates of giant gartersnakes, the manner in which survival depends on the size of the individual, the fecundity of adult female giant gartersnakes and the way this varies with female size, and the way the growth rate of giant gartersnakes changes throughout its life as it grows in size. Second, we used these three vital rates to construct an IPM that accurately captures the demography of giant gartersnake populations and the associated uncertainty with each underlying vital rate. Third, we used elasticity analysis of the IPM to measure how much the population growth rate of giant gartersnakes is affected by each vital rate. Finally, we developed a stochastic IPM that incorporates yearly variation in vital rates, which could be used for PVAs in the future. Our study represents the first complete demographic model for this threatened species. The results of the model provide researchers and managers with valuable information on where to direct their efforts to improve the understanding of giant gartersnake population biology and inform potential management actions that could increase the probability of persistence of populations.

\section{Methods}

\section{Data Sources}

We compiled data on the growth, reproduction, and survival of giant gartersnakes from three main sources (summarized in table 14): (1) Field studies of giant gartersnakes in the Sacramento Valley conducted by the U.S. Geological Survey from 1995 to 2016; (2) studies by Hansen and others (2015, 2017) of giant gartersnake fecundity, survival and growth in the Sacramento Valley and the San Joaquin Valley, from 2004 to 2016; and (3) published scientific literature on the demography of giant gartersnakes and other closely related species in the genus Thamnophis. Data from these different sources were integrated to construct an IPM for giant gartersnakes. As described below, we account for differences among studies or uncertainty in demographic parameters by either modeling separate IPMs or including the variation as an additional parameter in a stochastic model. 
Table 14. Vital rate models, parameters, and their primary and secondary sources.

[USGS, U.S. Geological Survey; CMR, capture-mark-recapture; SVL, snout-vent length; SD, standard deviation]

\begin{tabular}{|c|c|c|c|c|c|}
\hline $\begin{array}{c}\text { Demographic } \\
\text { process }\end{array}$ & Model & Parameter & Description & Primary source & Secondary source \\
\hline \multirow[t]{4}{*}{$\begin{array}{l}\text { Probability of } \\
\text { survival }\end{array}$} & \multirow[t]{4}{*}{$\begin{array}{l}p_{\mathrm{s}}(x)=\mathrm{c} * \operatorname{logit}^{-1}\left(\alpha_{\mathrm{s}}+\beta_{\mathrm{s} 1} * x+\right. \\
\left.\beta_{\mathrm{s} 2} * x^{2}\right)\end{array}$} & $\alpha_{\mathrm{s}}$ & Survival intercept & USGS CMR data & Hansen and others (2015) \\
\hline & & $\beta_{\mathrm{s} 1}$ & $\begin{array}{l}\text { Linear effect of size on } \\
\text { survival }\end{array}$ & USGS CMR data & Hansen and others (2015) \\
\hline & & $\beta_{\mathrm{s} 2}$ & $\begin{array}{l}\text { Quadratic effect of size on } \\
\text { survival }\end{array}$ & USGS CMR data & \\
\hline & & $\mathrm{c}$ & Survival ceiling & USGS CMR data & \\
\hline \multirow[t]{4}{*}{ Fecundity } & \multirow[t]{4}{*}{$\begin{array}{l}f_{\mathrm{n}}=\mathrm{p}_{\mathrm{g}}(x)^{*} \mathrm{p}_{\mathrm{f}} *(1- \\
\left.p_{s b}\right)^{*} p_{r e c}\left(\exp \left(\alpha_{\mathrm{f}}+\beta_{\mathrm{f}}^{*} x\right)\right)\end{array}$} & $\alpha_{\mathrm{f}}$ & Fecundity intercept & USGS reproductive data & $\begin{array}{l}\text { Hansen and others (2017), } \\
\text { Hansen and Hansen (1990) }\end{array}$ \\
\hline & & $\beta_{\mathrm{f}}$ & $\begin{array}{l}\text { Linear effect of size on } \\
\text { fecundity }\end{array}$ & USGS reproductive data & $\begin{array}{l}\text { Hansen and others (2017), } \\
\text { Hansen and Hansen (1990) }\end{array}$ \\
\hline & & $\mathrm{p}_{\mathrm{sb}}$ & Probability of stillbirth & USGS reproductive data & \\
\hline & & $\mathrm{p}_{\mathrm{f}}$ & $\begin{array}{l}\text { Proportion of female } \\
\text { offspring }\end{array}$ & USGS captive litters & \\
\hline \multirow[t]{2}{*}{$\begin{array}{l}\text { Probability of } \\
\text { being gravid }\end{array}$} & \multirow[t]{2}{*}{$\mathrm{p}_{\mathrm{g}}(x)=\operatorname{logit}^{-1}\left(\alpha_{\mathrm{g}}+\beta_{\mathrm{g}} * x\right)$} & $\alpha_{\mathrm{g}}$ & $\begin{array}{l}\text { Intercept for probability } \\
\text { of being gravid }\end{array}$ & Hansen and others (2017) & \\
\hline & & $\beta_{g}$ & $\begin{array}{l}\text { Slope for probability of } \\
\text { being gravid }\end{array}$ & Hansen and others (2017) & \\
\hline $\begin{array}{l}\text { Neonate } \\
\quad \text { recruitment }\end{array}$ & $p_{\text {rec }}$ & $\mathrm{p}_{\mathrm{rec}}$ & Probability of recruitment & USGS CMR data & $\begin{array}{l}\text { Literature on Thamnophis and } \\
\text { other Natricinae }\end{array}$ \\
\hline \multirow[t]{2}{*}{ Size at recruitment } & \multirow[t]{2}{*}{$x_{\mathrm{rec}} \sim N\left(\mu_{\mathrm{rec}}, \sigma_{\mathrm{rec}}\right)$} & $\mu_{\mathrm{rec}}$ & Mean recruit SVL & $\begin{array}{l}\text { USGS neonate size and growth } \\
\text { data }\end{array}$ & \\
\hline & & $\sigma_{\text {rec }}$ & SD recruit SVL & $\begin{array}{l}\text { USGS neonate size and growth } \\
\text { data }\end{array}$ & \\
\hline \multirow[t]{5}{*}{ Individual growth } & $x_{\mathrm{t}+1} \sim N\left(\mu_{\mathrm{t}+1}, \sigma_{\mathrm{x}}^{2}\right)$ & $\mathrm{L}_{\infty}$ & Asymptotic size & USGS growth data & \\
\hline & $\begin{array}{l}\mu_{\mathrm{t}+1}=x_{t}+\left(\mathrm{L}_{\infty}-\mathrm{x}_{\mathrm{t}}\right) *(1- \\
\quad \exp (-k * 365)\end{array}$ & $k$ & $\begin{array}{l}\text { Von Bertalanffy growth } \\
\text { coefficient }\end{array}$ & USGS growth data & \\
\hline & \multirow[t]{3}{*}{$\sigma_{\mathrm{x}}=\alpha_{\sigma}+\beta_{\sigma} * \mathrm{x}_{\mathrm{t}}$} & $\sigma_{x}^{2}$ & $\begin{array}{l}\text { Growth increment } \\
\text { variance }\end{array}$ & USGS growth data & \\
\hline & & $\alpha_{\sigma}$ & $\begin{array}{l}\text { Intercept of growth } \\
\text { increment SD }\end{array}$ & USGS growth data & \\
\hline & & $\beta_{\sigma}$ & $\begin{array}{l}\text { Slope of growth } \\
\text { increment SD }\end{array}$ & USGS growth data & \\
\hline
\end{tabular}




\section{Growth Kernel}

For our model, we used a snake's size (SVL) as the state variable. The growth kernel, $G\left(x^{\prime}, x\right)$, projects changes in a snake's size over time. Specifically, the growth kernel describes the probability of growing from size $x$ in year $t$ to size $x^{\prime}$ in year $\mathrm{t}+1$. To model the somatic growth trajectory of giant gartersnakes, we fit a von Bertalanffy growth function (eq. 4) to data on growth increments from snakes that were captured multiple times (section 1). We calculate the expected size of an individual at the next time step, $\mu_{\mathrm{t}+1}$, based on $x$, the initial size of the captured individual, $\mathrm{L}_{\infty}$; the asymptotic length; or the theoretical maximum length that females approach as they grow and age, $\Delta \mathrm{t}$, the time interval between $\mathrm{t}$

$$
\begin{gathered}
\mu_{t+1}=x+\left(L_{\infty}-x\right) *\left(1-e^{-k * \Delta t}\right) \\
x^{\prime} \sim N\left(\mu_{t+1}, \sigma_{x^{\prime}}^{2}\right)
\end{gathered}
$$

and $\mathrm{t}+1$ in days, and the growth coefficient, $k$, which determines how quickly an individual approaches its asymptotic size. A larger value of $k$ represents a faster growth rate. Because there is variability in growth among individuals, the actual size of an individual at time $\mathrm{t}+1, x^{\prime}$, is described by a normal distribution, with a mean equal to $\mu_{\mathrm{t}+1}$, the expected size, and a variance equal to $\sigma_{x^{\prime}}^{2}$. We included random effects of study site and year on $k$, and a random effect of study site on $\mathrm{L}_{\infty}$. We then used the year random effect to model temporal variation in giant gartersnake growth (see section "Stochastic IPM"). We then constructed the growth kernel, $G\left(x^{\prime}, x\right)$, using the mean and variance of the expected size in year $\mathrm{t}+1$ from the von Bertalanffy growth function. For more details on the estimation of the growth trajectory of giant gartersnakes, see section 1 .

\section{Survival Kernel}

The survival kernel, $S(x)$, defines the probability a snake survives from year $\mathrm{t}$ to year $\mathrm{t}+1$, which depends on the snake's SVL, $x$, in year t. The survival probability of a giant gartersnake is dependent on its size (Hansen and others, 2015; section 3). Thus the survival kernel is a logistic function with either a quadratic effect of snake SVL on survival (section 3 of this study; eq. 5) or a linear effect of snake SVL on survival (Hansen and others, 2015; eq. 6).

$$
\begin{gathered}
S(x)=c * \operatorname{logit}^{-1}\left(\alpha_{s}+\beta_{s 1} * x+\beta_{s 2} * x^{2}\right) \\
S(x)=\operatorname{logit}^{-1}\left(\alpha_{s}+\beta_{s 1} * x\right)
\end{gathered}
$$

In equations 5 and $6, \operatorname{logit}^{-1}$ is the logistic function, which translates the linear predictor on the right hand side of the equation back to the probability scale from 0 to 1 . Parameter $c$ is the survival ceiling $(<1), \alpha_{\mathrm{s}}$ is the survival intercept, $\beta_{\mathrm{s} 1}$ is the slope of the linear effect of snake SVL on survival, $\beta_{\mathrm{s} 2}$ is the slope of the quadratic effect of snake SVL on survival, and $x$ is the snake's SVL. For more details on how the size-survival relationships were estimated, please see section 3 and Hansen and others (2015). Because there were differences in the size-survival relationship between this study and Hansen and others (2015), we constructed separate IPMs using equation 5 ("USGS IPM") and equation 6 ("Hansen IPM"; see "Deterministic IPM" below for details). 


\section{Fecundity Kernel}

The fecundity kernel, $F\left(x^{\prime}, x\right)$ (eqs. 7-10), defines the number and size of offspring produced in year $\mathrm{t}+1$ by females in year $\mathrm{t}$, and is made up of three components.

$$
\begin{gathered}
p_{g}(x)=\operatorname{logit}^{-1}\left(\alpha_{g}+\beta_{g} * x\right) \\
f_{n}=p_{g}(x) *\left(1-p_{s b}\right) * p_{f} * p_{r e c} * \exp \left(\alpha_{f}+\beta_{f} * x\right) \\
x^{\prime} \sim N\left(\mu_{r e c}, \sigma_{r e c}^{2}\right) \\
F\left(x^{\prime}, x\right)=f_{n} * x^{\prime}
\end{gathered}
$$

The first component of the fecundity kernel (eq. 7) is the relationship between female SVL, $x$, and the probability a female is reproductive in a given year, $p_{g}(x)$, where $\alpha_{\mathrm{g}}$ is the intercept, and $\beta_{\mathrm{g}}$ is the slope of the size-probability of reproduction relationship. Not all adult female giant gartersnakes reproduce every year (section 2), and the probability a female is gravid is likely dependent on its size. For this component of the model, we used data and model results from female snakes that had their reproductive status determined by X-ray as part of the studies by Hansen and others (2017) of the reproductive biology of giant gartersnakes. Those studies modeled the probability a female was gravid using a Bayesian logistic regression, where the reproductive status of a female was coded as 1 if it was gravid and 0 if it was not gravid. Their model included a linear effect of female SVL on reproductive status, $\beta_{g}$, as well as linear and quadratic effects of the date of examination (not shown). Females were X-rayed from May through September, and female giant gartersnakes began giving birth in early July (section 2; Halstead and others, 2011b). Therefore, some of the examined females may not have been gravid because they already gave birth that year. We were interested in the probability a female reproduces at any point during the year, so we fixed the date of examination to the date at which the probability of finding evidence for reproduction is at its peak and then used the predicted relationship between female size and probability of reproduction, given the peak date. The size-reproductive status relationship was incorporated in the fecundity kernel, and this information was used, along with the litter size model in equation 8 , to project how many offspring are produced each year given the size distribution of females in the population.

The second component (eq. 8) is the relationship between female SVL, $x$, and the expected number of offspring produced by that female ( $f_{n}$, hereafter "fecundity"), given that the female is reproductive that year (from eq. 7). The fecundity of females was modeled as a function of their SVL, using data on embryo counts from X-rays, as well as captive-born litters from other USGS studies and one published study of giant gartersnake fecundity (Hansen and Hansen, 1990). We used a Bayesian Poisson regression to model the size-fecundity relation, which essentially models the log of the mean litter size as a linear function of the female's SVL. We used the intercept $\left(\alpha_{f}\right)$ and slope $\left(\beta_{f}\right)$ parameters from this regression to calculate the expected number of offspring produced each year, based on the size distribution of females in the population. This expected number of offspring was then multiplied by the proportion of offspring that are female $\left(p_{f}\right)$, which we estimated to be 0.51 on the basis of data from captive litters (section 2; Halstead and others, 2011b). Only female offspring were tracked because our demographic model was concerned only with the female population. The expected number of female offspring was then multiplied by $1-p_{s b}$, where $p_{s b}$ is the probability of stillbirth. Stillbirth is common in gartersnakes (Gregory and others, 1992) and has been observed in giant gartersnake litters (section 2). Because most of the fecundity data come from X-ray counts of embryos, it is important to adjust those embryo counts to account for stillbirths. Finally, the expected number of live female offspring at birth was multiplied by the probability of recruitment into the population, $p_{r e c}$, to give the expected number 
of live females that survive to 1 year old. This parameter takes into account neonate mortality before they enter the censused population at 1 year of age. More details on the recruitment parameter are described under the section "Probability of Recruitment."

The third component of the fecundity kernel (eq. 9) is the size distribution of snakes when they enter the population (hereafter, recruits). Because we generally surveyed giant gartersnake populations in the spring and early summer, before snakes give birth, we modeled fecundity using a prereproductive census (Ellner and others, 2016). In a pre-reproductive census, the population is censused right before offspring are born; therefore, individuals born immediately after the census are not included in the sample until the following year when they are 1 year old. Young snakes in our model recruit into the population (that is, join the modeled population) when they are 1 year old and thus have grown from their size at birth to a larger size in the intervening year. The size of recruits was modeled on the basis of the size of neonates at birth from captive-born snakes and the expected increase in SVL during the first year from our growth model (table 14). The size of recruits when they enter the modeled population, $x^{\prime}$, is a normal distribution with a mean equal to the average size of a 1-year-old snake, $\mu_{r e c}$, and a variance of $\sigma_{\text {rec }}^{2}$ which represents variation in the size of 1 -year-old snakes. Because snakes do not recruit into the population until they are 1 year old, we must model their survival from birth until recruitment as part of the fecundity kernel. This important parameter, $p_{r e c}$, is separate from the survival kernel, $S(x)$, used to model the survival process of snakes greater than or equal to 1 year of age that are already in the modeled population. Our method for estimating the probability of recruitment is described in section "Probability of Recruitment."

\section{Probability of Recruitment}

Estimating the survival rate of neonate reptiles is difficult (Pike and others, 2008). The survival rate of neonate giant gartersnakes during their first year, $p_{r e c}$, is an important component of our IPM because it determines how many of the offspring produced by females in year $t$ survive to enter the modeled population in year $\mathrm{t}+1$. Because most of the demographic sampling for giant gartersnakes is done in the spring and early summer before females give birth in early to mid-July (Halstead and others, 2011b; section 2), we do not capture and mark many neonate snakes (section 3). Because we do not have enough data from marked neonates, an indirect approach was used to estimate the probability of neonates surviving their first year and recruiting to the population. A robust-design Jolly-Seber CMR model was used that accounts for size-dependent capture probability to estimate the abundance, sex ratio, and size-distribution of giant gartersnakes at a site in a given year (Schofield and Barker, 2011). On the basis of the estimated size and abundance of all adult females (captured and unobserved females), and the size-fecundity and size-probability of reproduction relationships described above (under "Fecundity kernel"), we projected the expected number of neonate snakes produced in the population in year $\mathrm{t}, \widehat{N}_{n n, t}$. We then used the estimated size distribution and abundance of snakes the following year to estimate how many 1-year-old snakes of both sexes (captured and unobserved) are in

the population in year $\mathrm{t}+1, \widehat{N}_{r e c, t+1}$. On the basis of our growth model, we calculated the expected sizes of males and females in the late spring (the midpoint of sampling) of their first (1-year-old cohort) and second (2-year-old cohort) year. The expected size distribution of the 1-year-old cohort for a given sex was compared to the expected size distribution of the 2-year-old cohort for that sex, and we found the size threshold that best separates the range of smaller sizes that are more likely neonates than 1-year-old snakes from the range of larger sizes that are more likely 1-year-old snakes than neonates. Using this method, we defined females with less than $514 \mathrm{~mm} \mathrm{SVL}$ to be part of the 1-year-old cohort because 92 percent of 1-year-old females are expected to be less than $514 \mathrm{~mm} \mathrm{SVL}$; only 9 percent of 2-year-old females are expected to be less than $514 \mathrm{~mm}$ SVL, according to our growth model. For males the 
threshold to be part of the 1-year-old cohort is $457 \mathrm{~mm}$ SVL because 94 percent of 1-year-old males are expected to be less than $457 \mathrm{~mm} \mathrm{SVL}$, and 7 percent of 2-year-old males are expected to be less than $457 \mathrm{~mm}$ SVL. We then divided the total estimated number of 1-year-old snakes (males and females) in year $t+1$ by the expected number of neonates (of both sexes) produced in year $t$ to estimate the proportion of neonates that recruited to the population (eq. 11).

$$
p_{\text {rec }}=\frac{\widehat{N}_{r e c, t+1}}{\widehat{N}_{n n, t}}
$$

This analysis was restricted to data from four sites within the Natomas Basin that had enough captures for population estimation, and snakes were trapped consistently in the spring and early summer (pre-reproductive period) from 2011 to 2016 (Natomas 1-3 and 5 from section 3). Although we were interested only in females for our IPM, we used estimates of neonate and 1-year-old (recruit) abundance from both sexes to get a larger sample size and, therefore, a more precise estimate of $p_{\text {rec }}$. We assumed that survival during the first year is equal between males and females, and thus we used the estimates of $p_{\text {rec }}$ described above in our IPM to model survival of females from birth to 1 year old. The posterior distributions for estimates of the probability of recruitment are often right skewed with long tails. Therefore, for estimates of the probability of recruitment, we report the mode (under "Vital Rates" in the results of this section; calculated using the "modeest" package in R) because this measure is less affected by the skewness and long tail, and better reflects the region of highest posterior density.

We searched the scientific literature for studies that estimated the survival rate of neonates or probability of recruitment in other gartersnake species or snake species within the sub-family Natricinae, to which giant gartersnakes belong. We used our indirect estimates of the probability of recruitment and published estimates of neonate survival from closely related species to select a range of biologically realistic values for this parameter.

\section{Deterministic IPM}

Using the demographic models above, we built a deterministic IPM (eqs. 12-14),

$$
\begin{gathered}
n\left(x^{\prime}, t+1\right)=\int_{\Omega} K\left(x^{\prime}, x\right) n(x, t) d x \\
K\left(x^{\prime}, x\right)=P\left(x^{\prime}, x\right)+F\left(x^{\prime}, x\right) \\
P\left(x^{\prime}, x\right)=G\left(x^{\prime}, x\right) S(x)
\end{gathered}
$$

where $K\left(x^{\prime}, x\right)$ is the IPM kernel, which is the demographic model that combines models of the growth, $G\left(x^{\prime}, x\right)$, survival, $S(x)$, and fecundity $F\left(x^{\prime}, x\right)$ processes. The IPM kernel, $K\left(x^{\prime}, x\right)$, is analogous to the matrix in a matrix population model (for example, Caswell, 2001). The state of the population at time $\mathrm{t}$ is described by the size distribution, $n(x, t)$, which is multiplied by the kernel, $K\left(x^{\prime}, x\right)$, to give $n\left(x^{\prime}, t+1\right)$, the projected size distribution of the population at time $t+1$. This IPM is based on the mean parameter values estimated for the growth, survival, and fecundity of giant gartersnakes and therefore represents a hypothetical average giant gartersnake population (table 15). Because the size-survival relationships from USGS project data (section 3) and Hansen and others (2015) differed in shape, we constructed two separate IPMs. The first IPM was built with the quadratic size-survival function with a survival-ceiling less than 1 estimated from CMR models of USGS project data (section 3; eq. 5), which we refer to as the "USGS IPM." The second IPM was built with the linear size-survival function reported by Hansen and others (2015) (eq. 6), which we refer to as the "Hansen IPM." 
For each IPM, we calculated the stable size distribution, which is the expected size distribution a population would converge to, over time, if it grew (or declined) according to the demographic rates in the IPM. The stable size distribution provides a useful diagnostic tool of how well the IPM captures the demography of the study species, and is often compared to empirical size-distribution data to confirm that the IPM is not mis-specified (Easterling and others, 2000; Merow and others, 2014). The stable size distribution from each IPM was compared to empirical data on the size of female snakes captured from June 1-July 15 from 2013 to 2016 . We focused on captures from this time period because it represents the "pre-reproductive" period of the IPM and because we implemented new trapping methods in 2013 that increased our capture probability (section 3). Because capture probability varies as a function of snake size (section 3), the observed size-frequency distribution of snake captures was scaled by the capture probability for each size. By scaling the empirical size data, we accounted for the relationship between snake size and capture probability to produce a size-frequency distribution that better represents the true state of the population, rather than just the state of snakes that we were able to capture. We also calculated the relationship between snake size and reproductive value. Reproductive value is the present value of any future offspring produced by a female of size $x$ and is a function of the survival and fecundity kernels (Easterling and others, 2000; Caswell, 2001). Finally, we calculated the expected population growth rate, $\lambda$, for each IPM. A $\lambda$ equal to 1 represents a stable population, $\lambda$ less than 1 indicates a declining population, and $\lambda$ greater than 1 indicates a growing population.

We calculated the elasticity of $\lambda$ to changes in the IPM kernel. The elasticity of an IPM is the proportional change in $\lambda$ that results from a very small change in any of the size-dependent transition processes that make up the IPM (for example, the probability of survival for a snake that is $600 \mathrm{~mm}$ SVL). Elasticity is useful as a metric for evaluating IPMs because it scales the effects of a parameter or transition probability on $\lambda$ by the magnitude of that parameter or transition probability and thus diminishes the importance of highly improbable transitions (for example, growth from $400 \mathrm{~mm}$ SVL to $1,000 \mathrm{~mm}$ SVL in 1 year). A high elasticity value indicates that changing the probability of that sizetransition will have a large effect on the growth rate of the population (Easterling and others, 2000; Caswell, 2001). For example, if the survival rate of snakes that are $600 \mathrm{~mm}$ SVL has a high elasticity, it means that if the survival rate of individuals of that size could somehow be increased, the population is expected to show a large increase in the population growth rate. In the "Deterministic IPM" section of the results below, we present the USGS and Hansen IPMs and compare their elasticities to determine whether the shape of the size-survival relationship affects our inferences from these models. We also calculated the elasticity of $\lambda$ from changes in the parameters of the underlying vital rate models (for example, survival, growth, and fecundity) following the methods of Rees and Rose (2002). For each vital rate parameter $p$, (for example, the slope of the size-fecundity relation), we increased its value by 1 percent over the mean, constructed a new IPM, calculated the population growth rate $\lambda_{2}$, and compared it to the original $\lambda_{1}$. We then calculated the elasticity of $\lambda$ to the change in the vital rate parameter using equation 15 , where $\Delta \lambda=\lambda_{2}-\lambda_{1}$.

$$
\operatorname{elasticity}(p)=\frac{\Delta \lambda}{\Delta p} * \frac{p}{\lambda}
$$


Table 15. Demographic model parameters and estimates of their mean and standard deviation for giant gartersnakes in Sacramento Valley, California.

[Abbreviations are defined in table X. SD, standard deviation; --, no data]

\begin{tabular}{|c|c|c|c|c|}
\hline Demographic process & Model & Parameter & Mean & SD \\
\hline \multirow[t]{4}{*}{ Probability of survival } & \multirow[t]{4}{*}{$p_{\mathrm{s}}(x)=\mathrm{c} * \operatorname{logit}^{-1}\left(\alpha_{\mathrm{s}}+\beta_{\mathrm{s} 1} * x+\beta_{\mathrm{s} 2} * x^{2}\right)$} & $\alpha_{\mathrm{s}}$ & 0.18 & 0.77 \\
\hline & & $\beta_{\mathrm{s} 1}$ & 0.61 & 0.54 \\
\hline & & $\beta_{\mathrm{s} 2}$ & -0.26 & 0.29 \\
\hline & & $\mathrm{c}$ & 0.85 & 0.12 \\
\hline \multirow[t]{4}{*}{ Fecundity } & \multirow{4}{*}{$f_{\mathrm{n}}=\mathrm{p}_{\mathrm{g}}(x)^{*} \mathrm{p}_{\mathrm{f}}^{*}\left(1-p_{s b}\right)^{*} p_{r e c^{*}}\left(\exp \left(\alpha_{\mathrm{f}}+\beta_{\mathrm{f}}^{*} x\right)\right)$} & $\alpha_{\mathrm{f}}$ & 0.83 & 0.29 \\
\hline & & $\beta_{\mathrm{f}}$ & 0.0025 & 0.0004 \\
\hline & & $\mathrm{p}_{\mathrm{sb}}$ & 0.18 & 0.02 \\
\hline & & $\mathrm{p}_{\mathrm{f}}$ & 0.51 & 0.04 \\
\hline \multirow[t]{2}{*}{ Probability of being gravid } & \multirow[t]{2}{*}{$\mathrm{p}_{\mathrm{g}}(x)=\operatorname{logit}^{-1}\left(\alpha_{\mathrm{g}}+\beta_{\mathrm{g}} * x\right)$} & $\alpha_{\mathrm{g}}$ & 0.23 & 1.13 \\
\hline & & $\beta_{g}$ & 1.81 & 0.39 \\
\hline Neonate recruitment & $p_{\text {rec }}$ & $p_{\text {rec }}$ & $0.1-0.4$ & -- \\
\hline \multirow[t]{2}{*}{ Size at recruitment } & \multirow[t]{2}{*}{$x_{\mathrm{rec}} \sim N\left(\mu_{\mathrm{rec}}, \sigma_{\mathrm{rec}}^{2}\right)$} & $\mu_{\mathrm{rec}}$ & 461 & -- \\
\hline & & $\sigma_{\text {rec }}$ & 62.8 & -- \\
\hline \multirow[t]{4}{*}{ Individual growth } & $x_{\mathrm{t}+1} \sim N\left(\mu_{\mathrm{t}+1}, \sigma_{\mathrm{x}}^{2}\right)$ & $\mathrm{L}_{\infty}$ & 910 & 22.5 \\
\hline & $\mu_{\mathrm{t}+1}=x_{t}+\left(\mathrm{L}_{\infty}-\mathrm{x}_{\mathrm{t}}\right) *(1-\exp (-k * \Delta t))$ & $k$ & 0.0013 & 0.00025 \\
\hline & \multirow[t]{2}{*}{$\sigma_{x}=\alpha_{\sigma}+\beta_{\sigma} * x_{t}$} & $\alpha_{\sigma}$ & 71.4 & -- \\
\hline & & $\beta_{\sigma}$ & -0.06 & -- \\
\hline
\end{tabular}

\section{Stochastic IPM}

The growth, survival, and fecundity of animals varies over time, and much of this variation may be stochastic (that is, random) in nature. A deterministic IPM that is based on mean parameter values for these vital rates may not fully encapsulate the range of real dynamics observed in wild populations (Mills and others, 1999). Also, the stochastic variation in growth, survival, and fecundity found in nature will, in general, decrease the expected long-term growth rate of a population and therefore increase the probability of a population going extinct (Ellner and others, 2016). Demographic population models, including IPMs and matrix models, are often used as part of a demographic PVA to project the relative probability of a population going extinct or persisting in the near future under different management or conservation scenarios. Therefore, if wildlife and resource managers use a deterministic IPM, which does not account for variation in vital rates over time, to project a population's trajectory, they could draw overly optimistic conclusions about the chances that a population will persist in the future. Because conducting a PVA is one of the designated recovery actions in the U.S. Fish and Wildlife Service's recovery plan for the giant gartersnake (U.S. Fish and Wildlife Service, 2017), we developed a stochastic IPM that could be used for this recovery action in the future.

To model the temporal variation in giant gartersnake demography, we constructed a stochastic IPM using the parameter selection method (Ellner and others, 2016). In the parameter selection method, vital rates such as individual growth, survival, and fecundity are randomly generated for a 
chosen number of simulated years. For each simulated year, growth, survival, and fecundity parameters are randomly drawn from a normal distribution on the basis of their mean value and the standard deviation of annual variation in that parameter, estimated from a hierarchical model (that is, based on the results in sections 1-3). The yearly random effect was used for each parameter to isolate the process variation (that is, how much does survival vary from year to year) from sampling variation (that is, uncertainty in the estimates of survival for a given year, as a result of finite sample sizes). We simulated annual variation in the growth coefficient, $k$ (that is, the growth rate of individuals), the intercept of the size-fecundity relation, $\alpha_{f}$ (that is, the average fecundity of a female), the intercept of the size probability of the reproduction relation, $\alpha_{\mathrm{g}}$ (that is, the average probability of reproduction), and the intercept of the size-survival relation, $\alpha_{s}$ (that is, the average survival rate) (table 16). All other vital rate parameters (for example, the slope of the size-survival relation, $\beta_{\mathrm{s} 1}$, or size-fecundity relation, $\beta_{\mathrm{f}}$ ) were held constant at their mean values used in the deterministic IPM. We then constructed an IPM using that year's growth, survival, and fecundity kernels and calculated the onestep population growth rate, $\lambda_{t}$, on the basis of that particular year's IPM. This process was repeated for 10,000 simulated years, constructing a different IPM for each year. We then calculated the expected long-term stochastic growth rate of the population, $\lambda_{s}$, which better reflects the long-term fate of the population under stochastic variation in vital rates than the value of $\lambda$ from a deterministic IPM. We also calculated the elasticity of $\lambda_{s}$ to perturbations of the stochastic IPM kernel and compared the elasticity of the stochastic IPM to the elasticity of the deterministic IPM. Comparing the stochastic and deterministic models allows us to see whether including temporal variation in vital rates affects which vital rates appear most important for the growth of the population. Because the deterministic USGS and Hansen IPMs were very similar (see section "Deterministic IPM" below), we constructed stochastic IPMs that were primarily based on the USGS project vital rate data only.

Correlations between vital rate parameters (for example, survival and individual growth rates might be positively correlated such that they are both higher in "good" years and lower in "bad" years) can be important to include in stochastic population models because they can affect the conclusions from elasticity analyses (Doak and others, 2005). We did not include covariance among vital rate parameters in the stochastic model because concurrent data from the same sites in the same years were not always available (for example, the fecundity data comes from different sources than the survival and growth data). For the vital rates that were estimated from the same sites over the same time period, we did not find evidence of a correlation between annual growth and survival, nor between female fecundity and the probability a female was reproductive.

Table 16. Mean and annual standard deviation of time-varying parameters used in the stochastic Integral Projection Model.

[SD, standard deviation; Pr, probability]

\begin{tabular}{llll}
\hline Parameter & \multicolumn{1}{c}{ Description } & Mean & SD(t) \\
\hline$\alpha_{\mathrm{f}}$ & Fecundity intercept & 0.83 & 0.24 \\
$k$ & Von Bertalanffy growth coefficient & 0.0013 & 0.0004 \\
$\alpha_{\mathrm{s}}$ & Survival intercept & 0.18 & 1.07 \\
$\alpha_{\mathrm{g}}$ & Intercept for Pr(gravid) & 0.23 & 1.45 \\
\hline
\end{tabular}




\section{Results}

\section{Vital Rates}

The growth of giant gartersnakes is initially rapid and slows with age after individuals reach sexual maturity (fig. 17a). The yearly growth increments are much larger for young snakes than for older snakes, leading to a wide distribution of possible sizes in year $t+1$ for snakes at a small size in year $t$. In contrast, for snakes that are already large in year $t$, their expected growth increment is small, so the range of possible sizes in year $t$ is narrow as well, resulting in a narrow band of high probability at large sizes (fig. 17b). Essentially, once a snake is very large, it is likely to grow a very small amount each year, so its size in the next year is predictable: it will be close to its size in the current year.

The growth kernel (fig. 17b) is multiplied by the survival kernel (fig. 18a, c) to give the combined survival-growth kernel (fig. 18b, d). The survival kernel from the USGS project and the kernel based on Hansen and others (2015) differ in two key ways. First, the annual survival rate is higher for all sizes in Hansen and others (2015) than the estimated survival rate from the USGS project. Second, the survival rate increases linearly with size in Hansen and others (2015), whereas the survival kernel from the USGS project shows an initial increase in survival with size, a peak in survival for snakes between 600 and $800 \mathrm{~mm} \mathrm{SVL}$, and a slight decline in survival for snakes greater than approximately $800 \mathrm{~mm}$ SVL. In the survival-growth kernel, this translates to a peak in the probability of surviving and growing to a new size in the next year, shown as an ellipse of bright red in figure $18 \mathrm{~b}$. In contrast, because the survival probability only increases with size in the Hansen survival kernel, the survival-growth kernel shows a linearly increasing probability of a snake surviving and growing to a new size in the next year (fig. 18d). Note that because snakes recruit into the population at SVL greater than $400 \mathrm{~mm}$ SVL on average, the survival kernel determines only the survival rate of snakes of this size and larger, once they have recruited into the population. The survival of smaller neonate snakes during their first year is modeled as part of the fecundity kernel using the probability of recruitment parameter (eq. 8; table 14). 

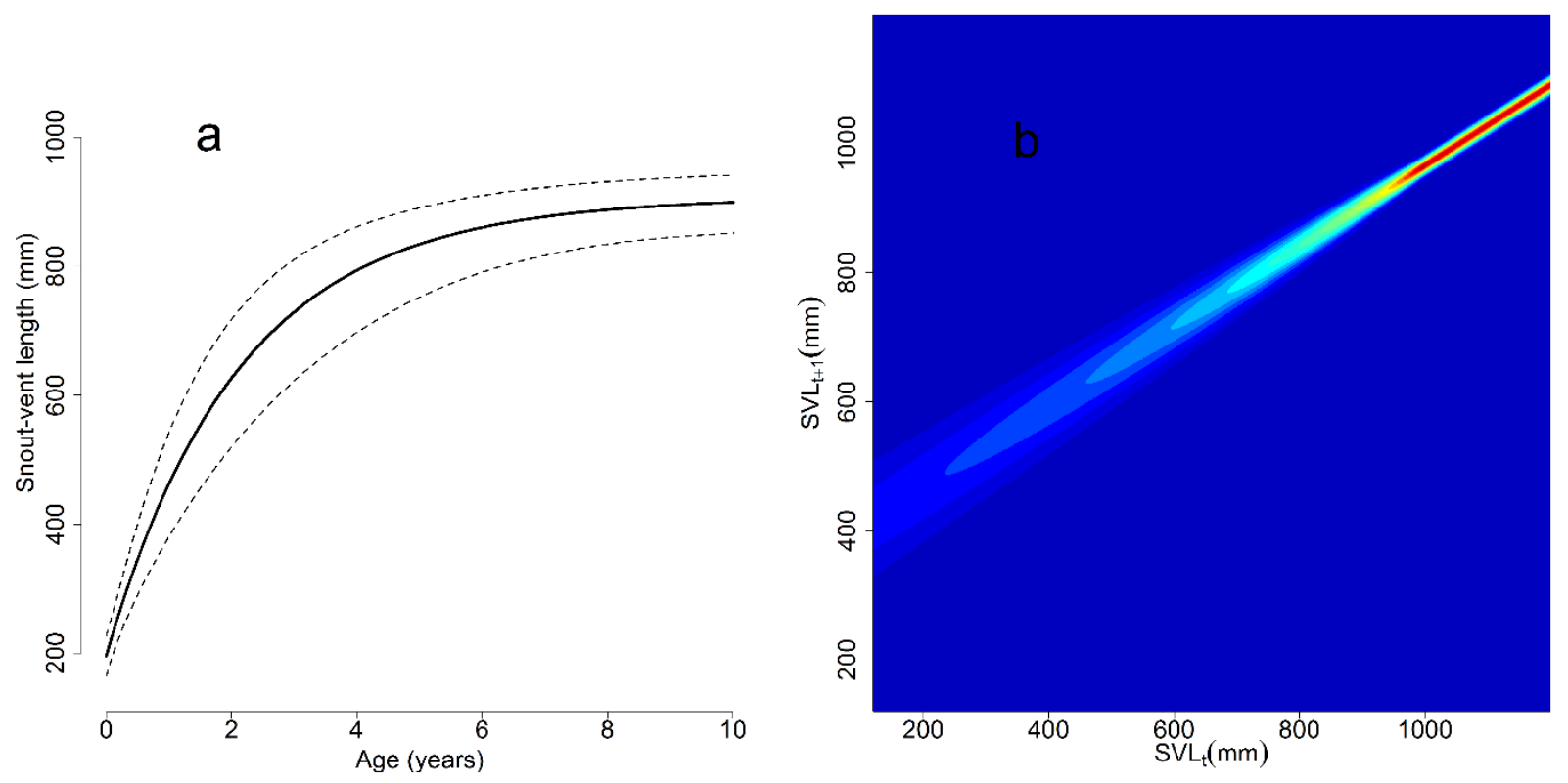

Figure 17. Growth trajectory and corresponding growth kernel for giant gartersnakes, Sacramento Valley, California. (a) von Bertalanffy growth curve for female giant gartersnakes. The solid line represents the mean growth curve, and the dashed lines show the 95-percent credible interval around the mean. (b) The growth kernel used in the giant gartersnake Integral Projection Model to project the snout-vent length (SVL) of snakes in year $\mathrm{t}+1$ on the basis of $\mathrm{SVL}$ in year t. Cool colors represent low probability; warm colors represent high probability. The variance in size decreases as snakes get longer. ( $\mathrm{mm}$, millimeter) 

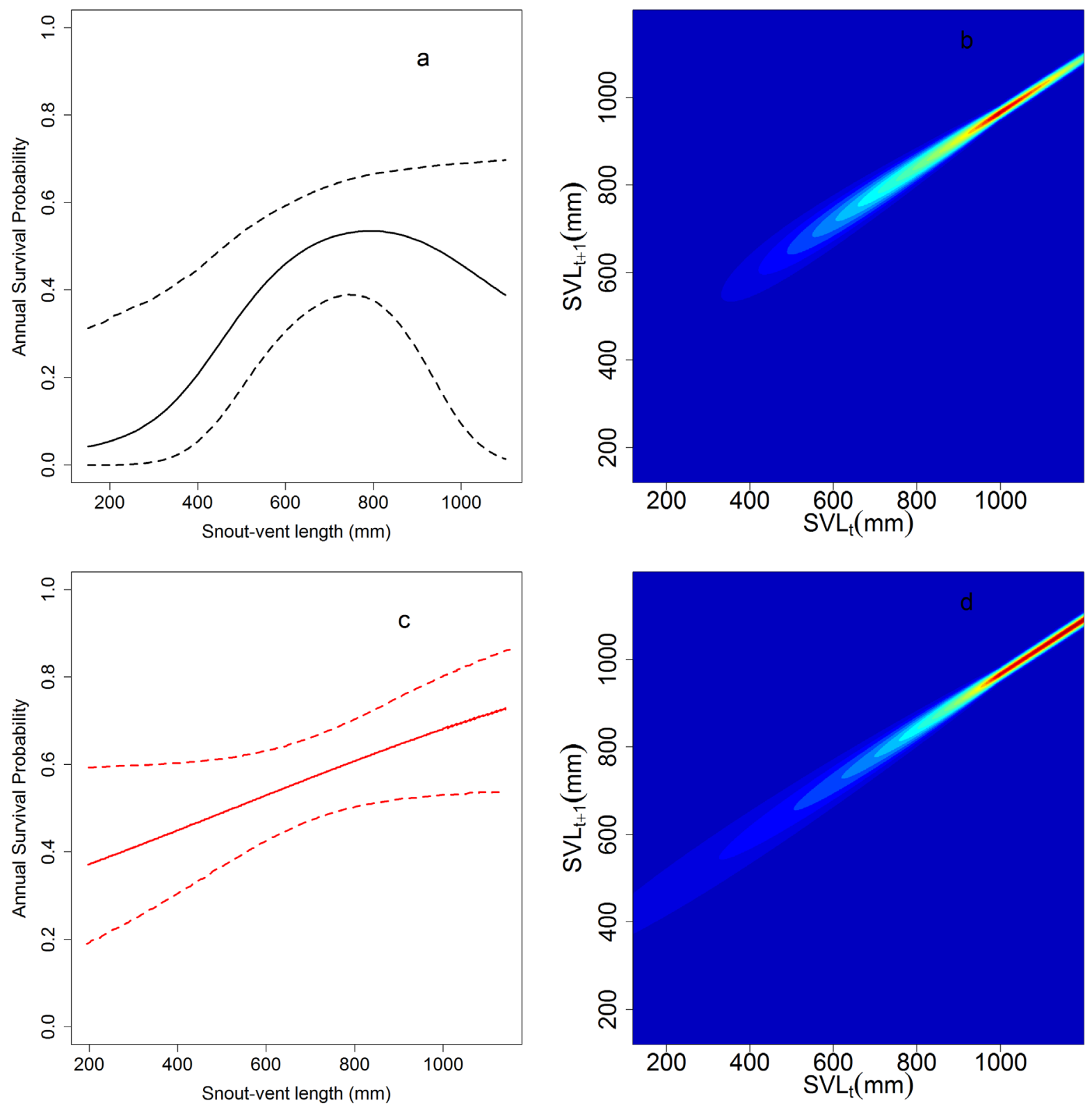

Figure 18. Size-survival relationship and survival kernel for giant gartersnakes, Sacramento Valley, California. (a) Size in relation to survival probability from U.S. Geological Survey project data. (b) Combined survival-growth kernel using U.S. Geological Survey quadratic size-survival function. (c) Size in relation to survival probability from Hansen and others (2015). (d) Combined survival-growth kernel using Hansen and others (2015) linear size-survival function. Solid lines in panels (a) and (c) represent mean size-survival relations; dashed lines represent 95-percent credible (a) or confidence (c) intervals. SVL, snout-vent length; mm, millimeter) 
The number of offspring produced and the probability a female is gravid increased with a female's SVL (fig. 19a, b). For the purposes of our IPM, the fecundity model projects the expected number of female offspring that survive to year $\mathrm{t}+1$, on the basis of the size of the mother in year $\mathrm{t}$. Thus, although litter sizes of giant gartersnakes are on average 15-23 neonate snakes (Hansen and Hansen, 1990; Halstead and others, 2011b; section 2), only one-half of those neonates are female, nearly 20 percent are likely to be stillborn (section 2), and likely fewer than one-half of those neonates survive until they are 1 year old. Therefore, the number of 1-year-old female snakes that recruit into the population is much smaller than the total litter size produced by an adult female. The relationship between the SVL of an adult female and the expected number of female recruits it produces is very similar for the USGS project and Hansen datasets (fig. 19a). As a result, the fecundity kernels for the two data sources are also very similar (fig. 19c, d). The fecundity kernels represent the number of recruits produced and the size of those recruits. Thus, the probability of producing offspring in the fecundity kernel is highest (red) for large adult females because they have the highest probability of being gravid and produce the largest litters. The variation in the color of the fecundity kernel along the $y$-axis represents the size distribution of recruits when they enter the population at 1 year old. Giant gartersnakes grow rapidly during their first year, so there can be a great deal of variance in growth among individuals, and the size distribution of 1-year-old recruits is much wider than the size distribution of neonates (section 2).

The probability of recruitment, $p_{\text {rec }}$, varied among populations and among years, and there was high uncertainty for many years, reflected in the long tails on the right side of the posterior distribution (fig. 20). The mode of the posterior distribution $p_{\text {rec }}$ varied from 0.09 to 0.23 at the Natomas 1 site, 0.05 to 0.44 for Natomas 2, 0.07 to 0.23 for Natomas 3, and 0.10 to 0.19 for Natomas 5. Our estimates of $p_{r e c}$ are lower than the predicted survival of an average neonate of $196 \mathrm{~mm} \mathrm{SVL}$ (0.37), estimated from the size-survival relationship for giant gartersnakes reported by Hansen and others (2015). Our estimates are also slightly lower than those reported from other gartersnakes and snakes in the subfamily Natricinae. Pike and others (2008) estimated the probability of neonate survival to be 0.3 for $T$. sirtalis (common gartersnake), 0.32 for T. ordinoides (northwestern gartersnake), 0.35 for T. proximus (western ribbonsnake), and 0.24 for Nerodia sipedon (northern watersnake). Similar values were reported from other studies of neonate survival in common gartersnakes (0.29) and northern watersnakes (0.31) (Jayne and Bennett, 1990; Kissner and Weatherhead, 2005). The probability of neonate survival ranged from approximately 0.28 to 0.43 in fast-growth populations of T. elegans (western terrestrial gartersnake; Miller and others, 2011), which likely have a life history similar to that of giant gartersnakes (see section 1). Estimates of first year survival were slightly lower for a population of T. radix (plains gartersnake; 0.16, 95-percent confidence interval 0.07-0.33) in Illinois (Stanford and King, 2004). Because our estimates of the probability of recruitment for giant gartersnakes were based on a new, indirect method and were lower than the values reported from studies of other closely related snake species, we chose to use four values for $p_{\text {rec }}(0.1,0.2,0.3,0.4)$ in our IPM analysis (tables 15,17 ). Where the results of the IPM analysis vary with the value of $p_{\text {rec }}$, we highlight and discuss those patterns, and where the value of $p_{\text {rec }}$ does not appear to affect results, we present results from an IPM with $p_{\text {rec }}$ set to 0.3 . 

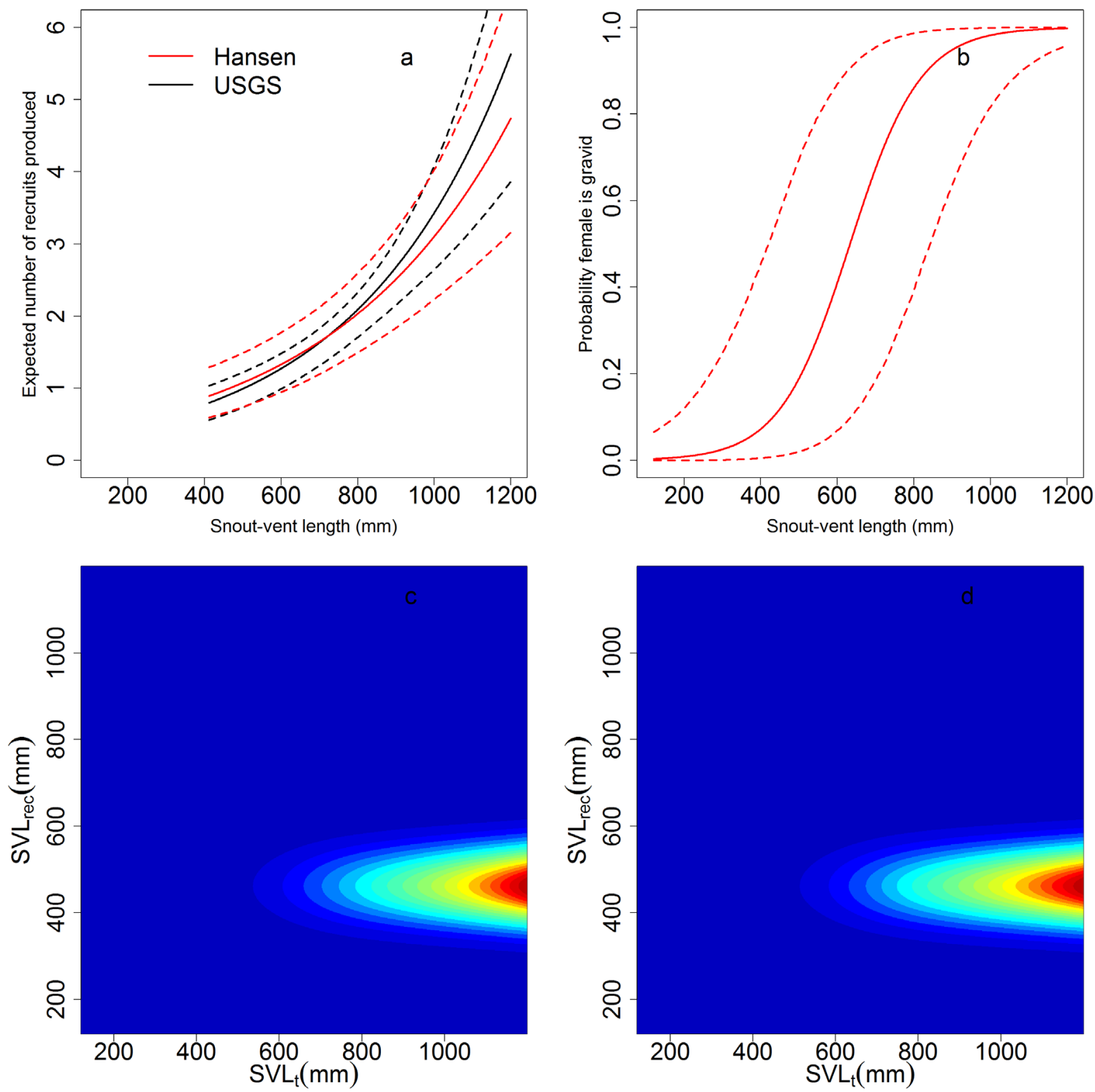

Figure 19. The fecundity kernel and its component models for giant gartersnake, Sacramento Valley, California. (a) Female snout-vent length (SVL) in relation to the expected number of offspring that will recruit to the population in year $t+1$. Black lines represent USGS project data, red lines represent data from Hansen and others (2017). (b) Female SVL in relation to the probability a female is gravid in a given year. (c) Fecundity kernel from USGS project fecundity data. (d) Fecundity kernel from Hansen and others (2017) data. In panels (c) and (d), the $y$-axis represents the SVL of 1-year-old recruits in year $t+1$, and the $x$-axis represents the SVL of adults present in the population in year t. (USGS, U.S. Geological Survey; mm, millimeter.) 

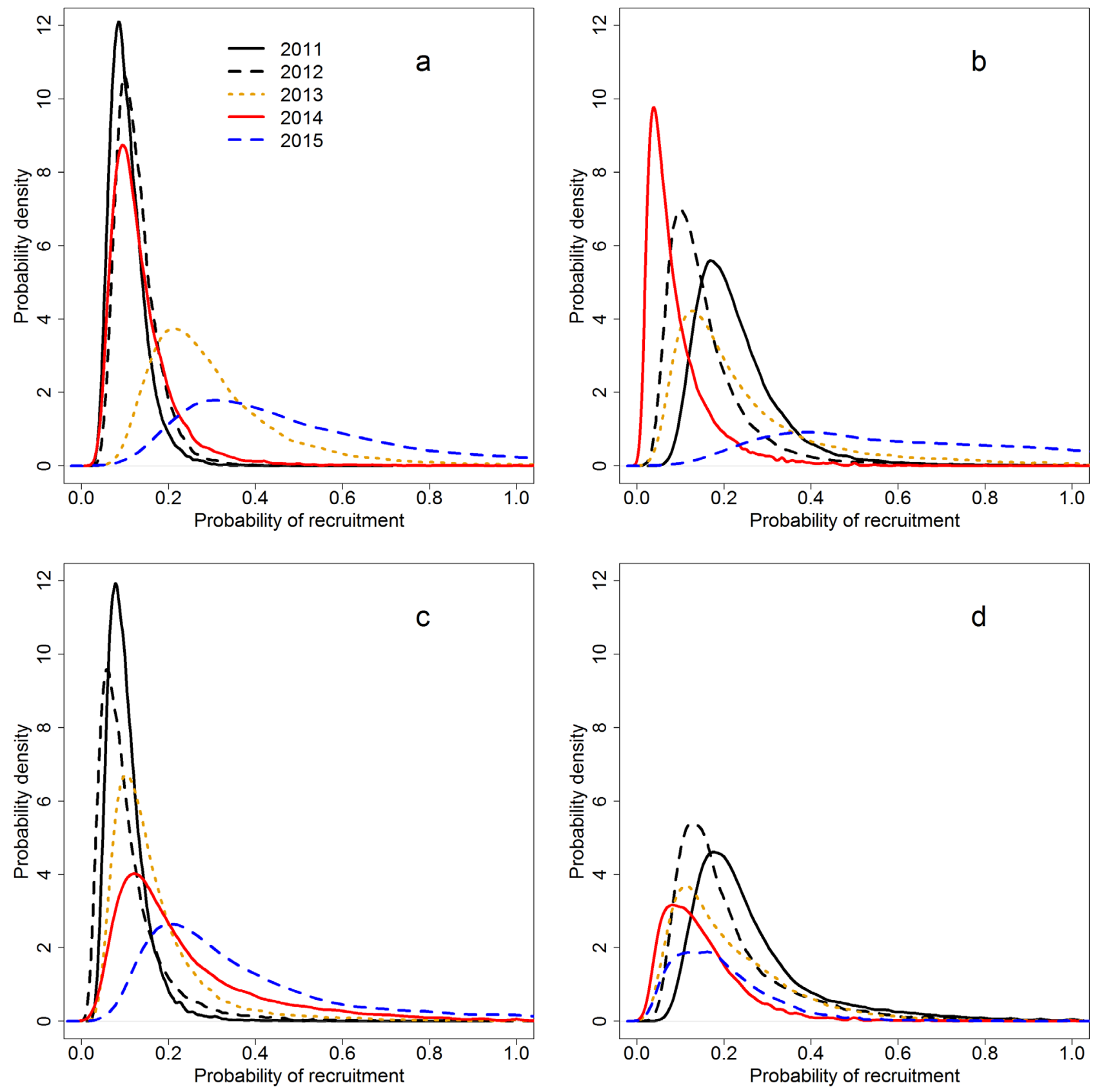

Figure 20. Estimated probability of recruitment for neonate giant gartersnakes from 2011 to 2015 at four sites in the Natomas Basin: (a) Natomas 1, (b) Natomas 2, (c) Natomas 3, (d) Natomas 5, Sacramento Valley, California. 


\section{Deterministic IPM}

The vital rate parameters used to create the deterministic IPMs are summarized in table 15 . The IPMs built using primarily USGS project data (USGS IPM; fig. 21a) or data from Hansen and others (2015, 2017) (hereafter Hansen IPM; fig. 21b) are similar. Both have two main features. (1) A narrow band runs from the bottom left of the figure to the upper right. This band (the "survival-growth region") represents the survival and growth of individuals present in the population in year $t$ in relation to their new size in year $\mathrm{t}+1$ and is analogous to the sub-diagonal elements in a matrix population model. It represents the combined survival-growth kernel, $P\left(x^{\prime}, x\right)$. (2) The half-ellipse along the lower right hand side of the figure is the fecundity kernel, $F\left(x^{\prime}, x\right)$. This "recruitment region" represents the production of new recruits in year $t+1$, based on the number of gravid adult females in year $t$, and is analogous to the fecundity parameters along the top row of a matrix population model. The IPM kernels from the two datasets are similar, except the probability of survival for very large snakes is higher in the Hansen IPM band (red color in the upper right portion of the diagonal, survival-growth band) than in the USGS band (yellow-green color in this region). Also the production of recruits is higher for very large females in the USGS IPM (red color in right-most section of the fecundity halfellipse) than in the Hansen IPM, which reflects the steeper slope in the size-fecundity relationship from the USGS project data (fig. 19a). One of the main outputs from an IPM is an estimate of the expected growth rate of the population, $\lambda$, based on the survival, growth, and fecundity of the individuals in the population. Values of $\lambda$ greater than 1 represent a growing population, $\lambda$ equal to 1 represents a stable population, and $\lambda$ less than 1 represents a declining population. In general, the USGS IPM represents a population that would decline over time, except when the probability of recruitment is very high $\left(\lambda=0.74,0.87,0.97\right.$, and 1.06 for $p_{r e c}=0.1,0.2,0.3$, and 0.4 , respectively). The Hansen IPM with its higher annual survival, especially for large adult females, represents a growing population in most scenarios, except when the probability of recruitment is very low $(\lambda=$ $0.88,1.03,1.16$, and 1.27 for $p_{\text {rec }}=0.1,0.2,0.3$, and 0.4 , respectively). For both IPMs, whether a population would be expected to grow or decline over time depends on the probability of recruitment, $p_{r e c}$. Because the true value of $p_{\text {rec }}$ is unknown, and because our IPMs integrate data from different populations at different points in time, the values of $\lambda$ reported above are not meant to be used to infer whether any particular population of giant gartersnakes is stable, growing, or declining, or to infer range-wide trends in the abundance of giant gartersnakes.

The stable size distribution predicted by both IPMs corresponds well with the captureprobability-adjusted size distribution of female snakes captured in June and early July (fig. 22a). Both IPMs predict that $400-500 \mathrm{~mm}$ SVL is the most abundant size class in the population, followed by a second peak of snakes between 600 and $700 \mathrm{~mm}$ SVL. The empirical size-distribution also shows a peak between 400 and $480 \mathrm{~mm}$ SVL, but the second peak occurs at smaller sizes (500-550 mm SVL) than the stable size distribution (fig. 22a). Reproductive value, or the present value of expected future reproduction for a female of a given size, increases with female size in both IPMs. This pattern is expected in a species with highly size-dependent fecundity. Reproductive value increases exponentially with size in the Hansen IPM, whereas in the USGS IPM the slope of the sizereproductive value curve declines for the largest females, reflecting the lower survival for large adults in this model (fig. 22b). 

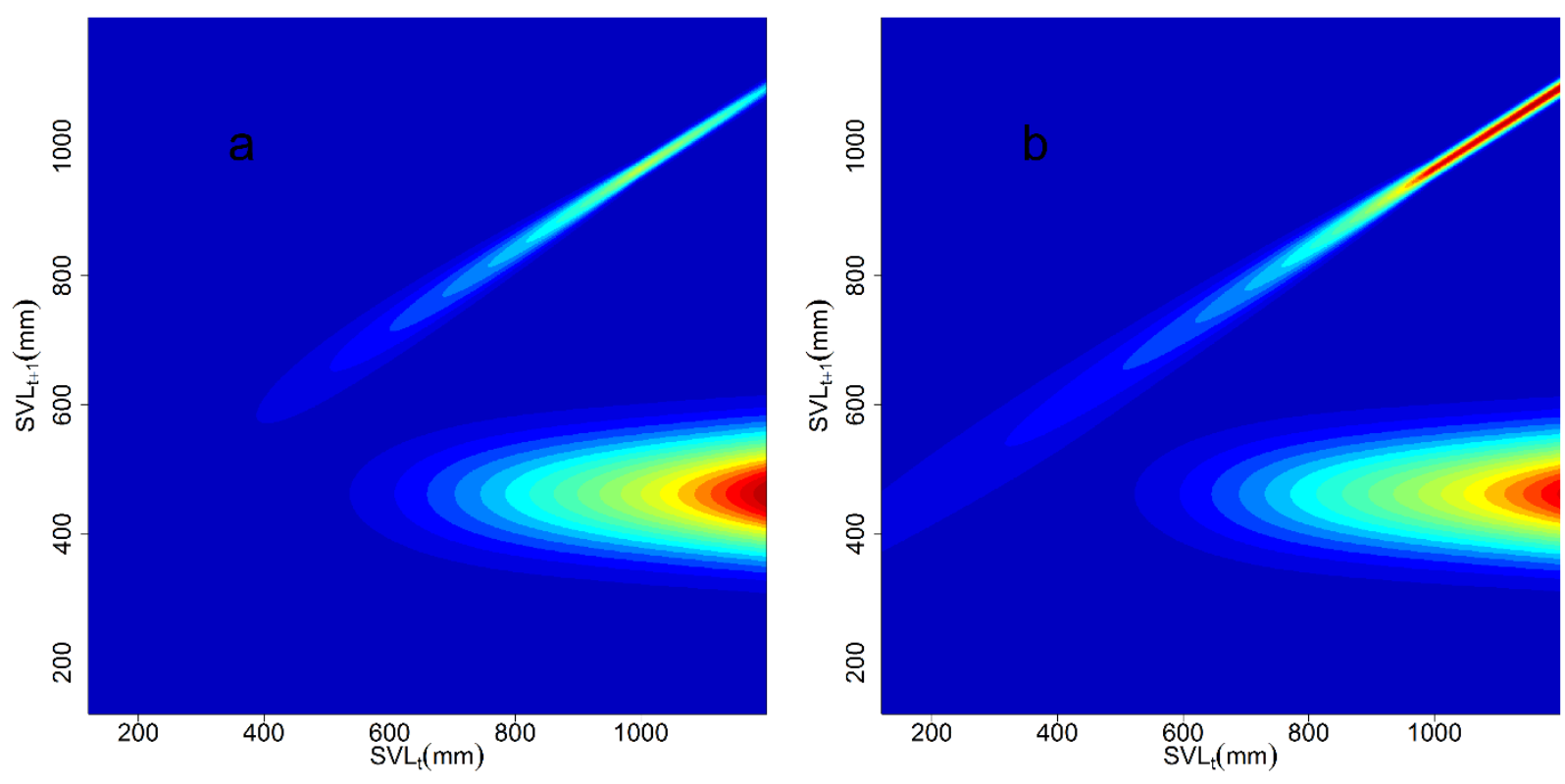

Figure 21. Deterministic Integral Projection Model kernels $\left(p_{\text {rec }}=0.3\right)$ for giant gartersnakes, Sacramento Valley, California. (a) The IPM kernel built using survival and fecundity data from U.S. Geological Survey project. (b). The IPM kernel built using survival and fecundity data from Hansen and others $(2015,2017)$. Each cell on the surface represents a probability of transitioning from one SVL in year $t$ to another SVL in year $t+1$. The half-ellipse in the lower right corner of the plots represents the fecundity component of the model, as small, new recruits in year $t+1$ are produced by large adult females. The narrow band in the center of the figure represents the survival and growth of individuals already present in the population in year t. Cool colors represent low probability; warm colors

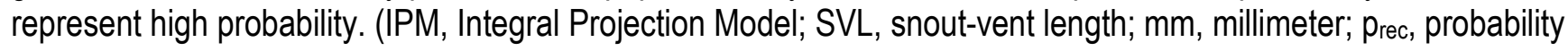
of recruitment.) 

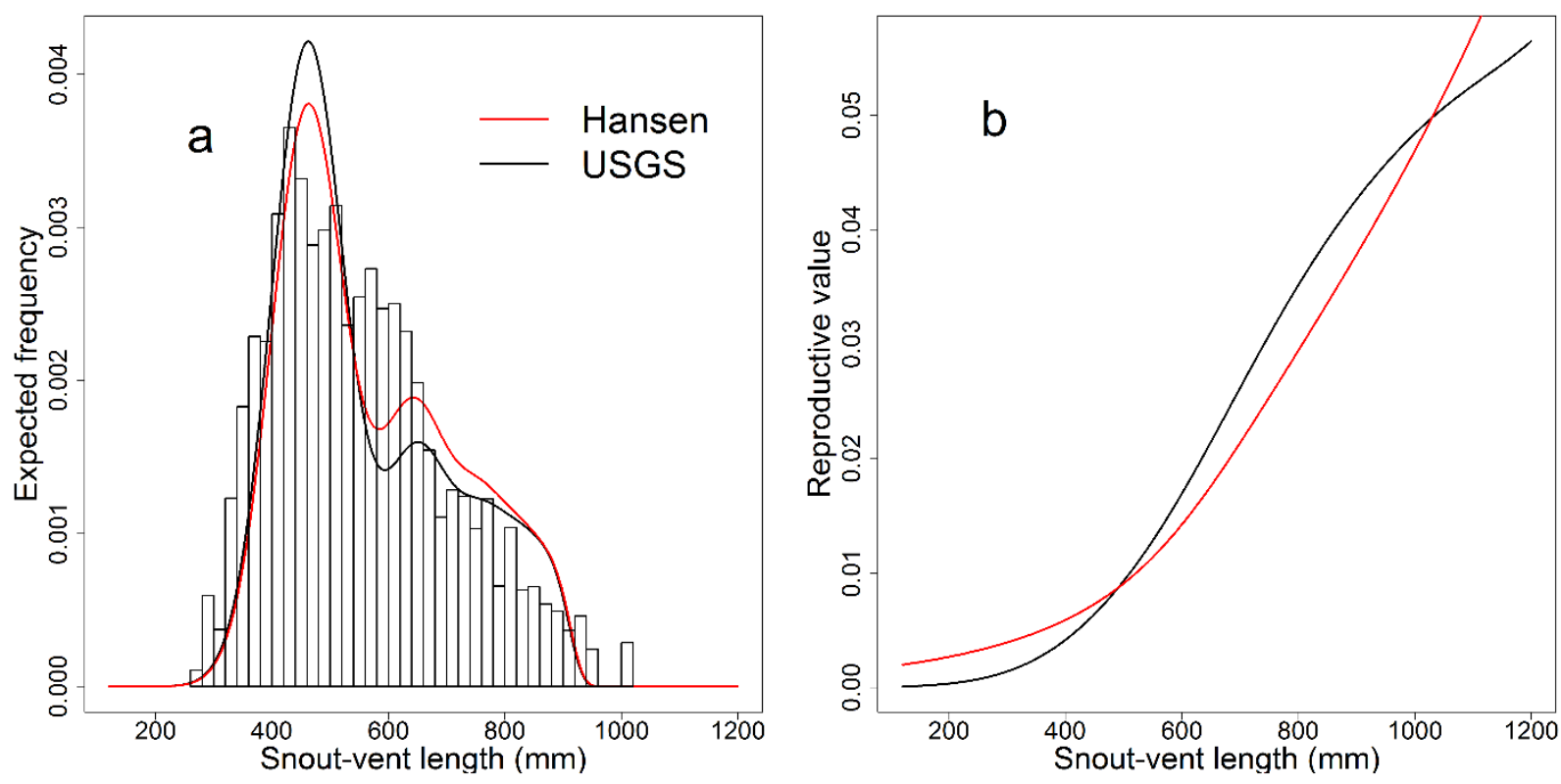

Figure 22. Deterministic Integral Projection Model diagnostics $\left(p_{\text {rec }}=0.3\right)$ for giant gartersnake, Sacramento Valley, California. (a) The stable size distribution (red and black lines) from the deterministic Integral Projection Models. The histogram represents the observed size frequency of female snakes captured between June 1 and July 1 from 2013 to 2016, scaled by the capture probability for each size. We scaled the observed captures for each size class to show the true size distribution in the population after accounting for the quadratic relationship between snake size and capture probability (see section 4 for details on the size-capture probability relation). (b) The reproductive value of an individual as a function of size. For both panels, black lines represent the USGS IPM and red lines represent the Hansen IPM. (USGS, U.S. Geological Survey; mm, millimeter; prec, probability of recruitment.)

Given the similarity of the IPMs built from each dataset, it follows that the elasticity surfaces calculated from each IPM are also very similar (figs. 23 and 24). Both IPMs show high elasticity for the survival and growth of large adult female snakes between approximately 700 and $900 \mathrm{~mm}$ SVL for all values of $p_{r e c}$, represented by an orange to red band in the upper right portion of the diagonal band. This means that increasing (or decreasing) the probability of survival for snakes in this size range has a very large effect on the population growth rate, $\lambda$. The relative importance of the survival of large adult females is greatest when $p_{\text {rec }}$ is low (figs. 23a, 24a). Each model also shows a secondary spot of high elasticity for the survival and growth of snakes from approximately $450 \mathrm{~mm} \mathrm{SVL}$ in year to greater than $600 \mathrm{~mm} \mathrm{SVL}$ in year $\mathrm{t}+1$. This spot represents the survival of females from 1 year to 2 years old and their growth into a size range that has much higher probability of reproducing. In essence, this region represents the growth and survival of young, immature females to an age and size at which they reach sexual maturity. The elasticity of $\lambda$ to the survival and growth of young, immature female snakes in this size range increases as $p_{\text {rec }}$ increases. Each IPM also shows a lighter region in the lower right hand area of the plot, which represents lower elasticity for the fecundity kernel, compared to the survival-growth kernel. The elasticity of this "recruitment region" increases as the value of $p_{\text {rec }}$ increases. This light-blue region indicates that increasing the probability of a female producing offspring of any one particular size at recruitment has a small effect on $\lambda$. As described below, the lower elasticity for this region of the IPM does not necessarily mean female fecundity is not important for determining population growth. 

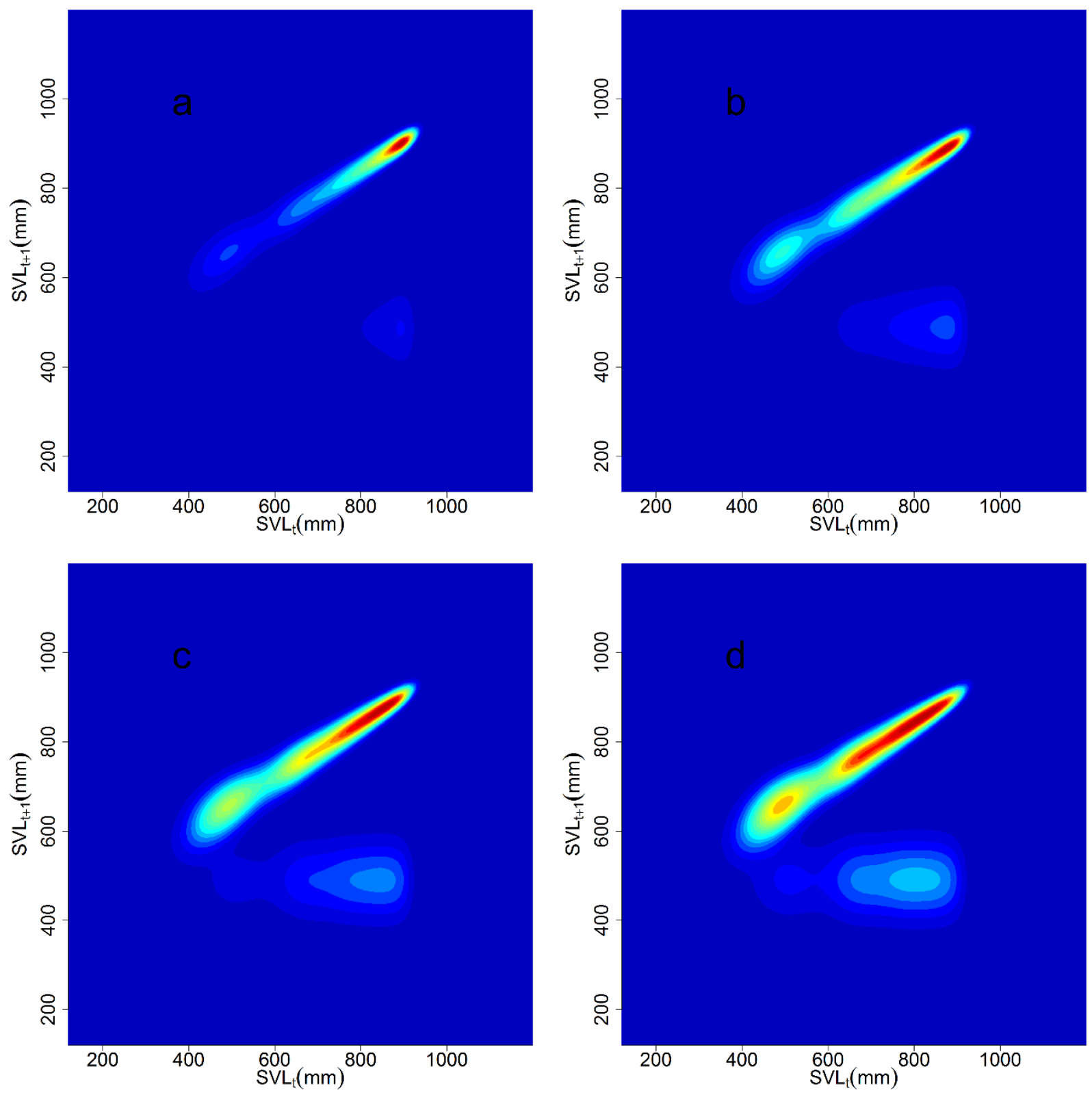

Figure 23. Elasticity surfaces for the U.S. Geological Survey deterministic Integral Projection Model based primarily on U.S. Geological Survey project data for giant gartersnakes, Sacramento Valley, California. Panels differ in the probability of recruitment: (a) $p_{\text {rec }}=0.1$, (b) $p_{\text {rec }}=0.2$, (c) $p_{\text {rec }}=0.3$, (d) $p_{\text {rec }}=0.4$. The value of each cell on the surface represents the proportional increase in the population growth rate, $\lambda$, from increasing that sizetransition probability. The light blue shape in the lower right represents the elasticity of $\lambda$ to changes in the fecundity components of the Integral Projection Model (IPM), and the diagonal band in the center represents the elasticity of $\lambda$ to changes in the survival-growth components of the IPM. Cool colors indicate increasing the transition probability has a small effect on $\lambda$; warm colors indicate increasing the transition probability has a large effect on $\lambda$. 

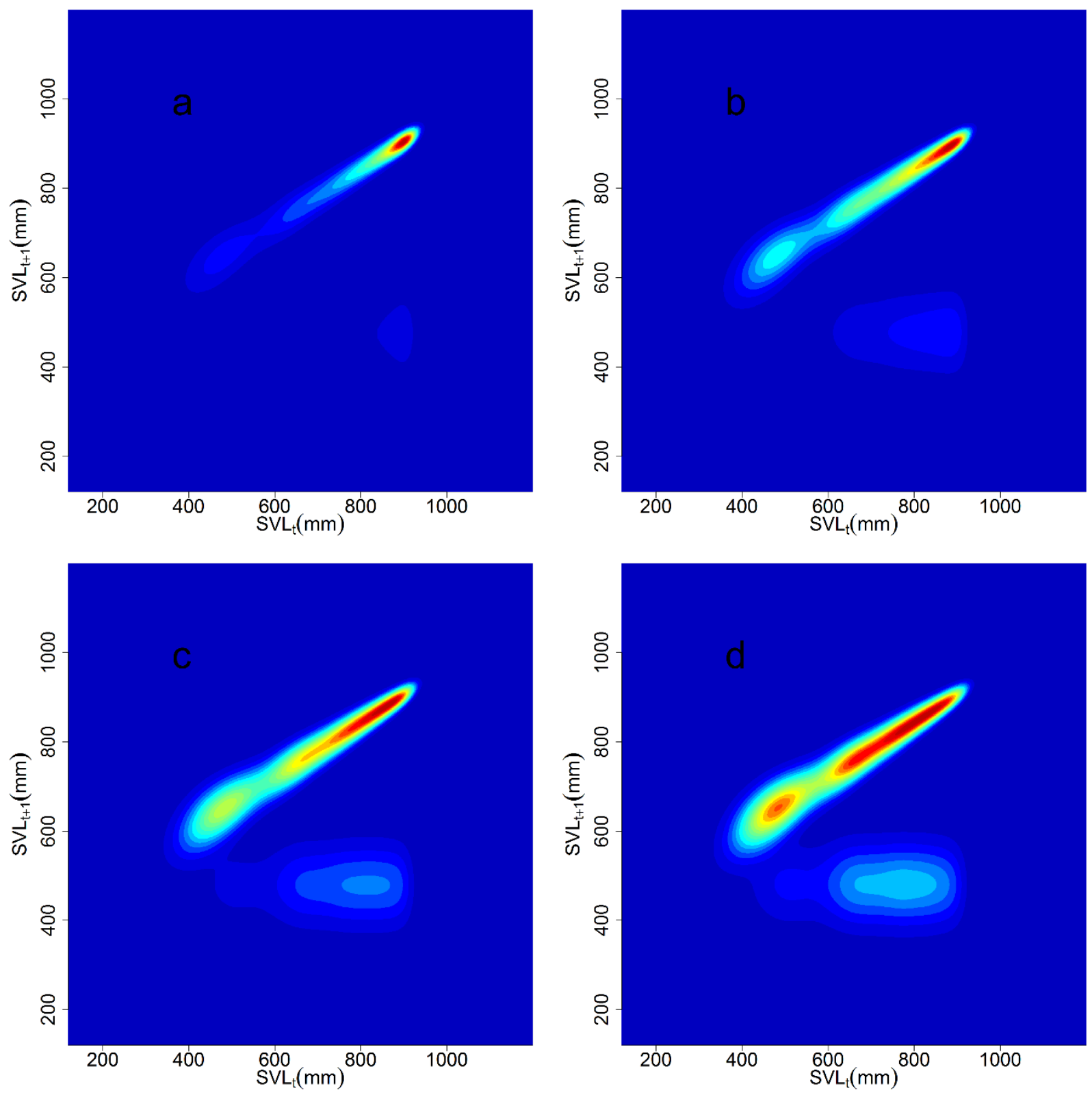

Figure 24. Elasticity surfaces for the "Hansen" deterministic Integral Projection Model based primarily on survival and reproductive data from Hansen and others (2017). Panels differ in the probability of recruitment: (a) $p_{\text {rec }}=$ 0.1 , (b) $p_{\text {rec }}=0.2$, (c) $p_{\text {rec }}=0.3$, (d) $p_{\text {rec }}=0.4$. The value of each cell on the surface represents the proportional increase in the population growth rate, $\lambda$, from increasing that size-transition probability. The light blue shape in the lower right represents the elasticity of $\lambda$ to changes in the fecundity components of the Integral Projection Model (IPM), and the diagonal band in the center represents the elasticity of $\lambda$ to changes in the survival-growth components of the IPM. Cool colors indicate increasing the transition probability has a small effect on $\lambda$; warm colors indicate increasing the transition probability has a large effect on $\lambda$. (SVL, snout-vent length; $\mathrm{mm}$, millimeter) 
The elasticity of population growth, $\lambda$, to changes in the vital rate parameters used to construct the IPM provides another picture of how different processes might affect giant gartersnake populations. The parameters that have the highest elasticity depend on the value of $p_{\text {rec }}($ tables 17,18$)$. The elasticity of survival parameters is negatively related to $p_{r e c}$; when $p_{\text {rec }}$ is low (and therefore $\lambda$ is also low), survival parameters (for example, $\alpha_{\mathrm{s}}, \beta_{\mathrm{s} 1}, \beta_{\mathrm{s} 2}$, and $c$ ) have their highest elasticity, and their elasticity decreases as $p_{r e c}$ and $\lambda$ increase. In contrast, the elasticity of vital rate parameters related to growth and especially fecundity (for example, $\mu_{\text {rec }}, L_{\infty}$, and $\beta_{\mathrm{f}}$ ) are positively related to $p_{\text {rec }}$ and $\lambda$. When the probability of recruitment is high and therefore population growth is greater, fecundity and growth have a greater proportional effect on $\lambda$.

In general, the four parameters with the highest elasticity for both IPMs, for all four scenarios of $p_{\text {rec }}$ are the mean size of neonates when they recruit into the population, $\mu_{\text {rec }}$; the asymptotic length of females, $\mathrm{L}_{\infty}$; the slope of the size-fecundity relation, $\beta_{f}$, and a survival parameter (the survivalceiling parameter $(c)$ for the USGS IPM and the slope of the size-survival relationship $\left(\beta_{s I}\right)$ for the Hansen IPM) (tables 17, 18). The larger the size of recruits when they enter the population at 1 year old, the higher the population growth rate. This is intuitive for two reasons. First, larger snakes have higher rates of survival. The larger an individual is when it is 1 year old, the higher its survival is during the following year. Second, a snake that enters the population at a larger size is likely to become reproductive sooner (that is, at a younger age than a small recruit) and thus contribute to the next generation. Interestingly, for all scenarios, the size at which recruits enter the population, $\mu_{\mathrm{rec}}$, has a much higher elasticity than $p_{\text {rec }}$, the probability of a neonate surviving to recruit into the population the following year. The importance of $\mathrm{L}_{\infty}$ is also intuitive: if the asymptotic size is larger, females can reach greater lengths and thus produce larger litters, which we would expect to increase the population growth rate. The slope of the size-fecundity relation, $\beta_{\mathrm{f}}$, and either the survival-ceiling parameter, $c$, (USGS IPM) or the slope of the size-survival relation, $\beta_{\mathrm{s} 1}$, (Hansen IPM) largely define the demographic rates of large adult female snakes and thus are related to the region of high elasticity depicted in figure 23a. Increasing the slope of the size-fecundity relationship means that larger females produce even larger litters. The production of more offspring by each adult female would be expected to increase population growth, whereas increasing the survival of large adult females increases the population growth rate because these individuals have the highest reproductive value. The slope and intercept for the relationship between female size and the probability of being gravid had very small elasticities for both IPMs under all four $p_{\text {rec }}$ scenarios, as did the standard deviation of SVL at recruitment (tables 17, 18). 
Table 17. Elasticity of the population growth rate, $\lambda$, of giant gartersnakes in Sacramento Valley, California, to perturbation of vital rate model parameters for the deterministic Integral Projection Model, based primarily on U.S. Geological Survey project data, for four values of the probability of recruitment, $p_{\text {rec}}$.

[All vital rate parameters were increased by 1 percent above their mean value, and the corresponding elasticity was calculated. We report the absolute value of elasticity. SVL, snout-vent length; SD, standard deviation; Pr, probability]

\begin{tabular}{|c|c|c|c|c|c|c|}
\hline \multirow{2}{*}{ Rank } & \multirow{2}{*}{ Parameter } & \multirow{2}{*}{ Description } & \multicolumn{4}{|c|}{$p_{\text {rec }}$} \\
\hline & & & 0.1 & 0.2 & 0.3 & 0.4 \\
\hline 1 & $\mu_{\mathrm{rec}}$ & Mean recruit SVL & 0.58 & 0.82 & 1.00 & 1.15 \\
\hline 2 & $\mathrm{~L}_{\infty}$ & Asymptotic size & 0.49 & 0.66 & 0.73 & 0.78 \\
\hline 3 & c & Survival ceiling & 0.80 & 0.74 & 0.70 & 0.67 \\
\hline 4 & $\beta_{\mathrm{f}}$ & Linear effect of size on fecundity & 0.39 & 0.48 & 0.54 & 0.58 \\
\hline 5 & $p_{\text {rec }}$ & Probability of recruitment & 0.20 & 0.26 & 0.30 & 0.33 \\
\hline 6 & $\mathrm{p}_{\mathrm{sb}}$ & Probability of stillbirth & 0.20 & 0.26 & 0.30 & 0.33 \\
\hline 7 & $\mathrm{p}_{\mathrm{f}}$ & Proportion of female offspring & 0.20 & 0.26 & 0.30 & 0.33 \\
\hline 8 & $\alpha_{\mathrm{f}}$ & Fecundity intercept & 0.16 & 0.21 & 0.25 & 0.27 \\
\hline 9 & $k$ & Von Bertalanffy growth coefficient & 0.12 & 0.17 & 0.20 & 0.22 \\
\hline 10 & $\beta_{\mathrm{s} 2}$ & Quadratic effect of size on survival & 0.11 & 0.09 & 0.07 & 0.07 \\
\hline 11 & $\alpha_{\mathrm{s}}$ & Survival intercept & 0.06 & 0.06 & 0.06 & 0.06 \\
\hline 12 & $\sigma_{\text {rec }}$ & SD recruit SVL & 0.01 & 0.03 & 0.04 & 0.05 \\
\hline 13 & $\beta_{\mathrm{g}}$ & Slope for $\operatorname{Pr}$ (gravid) & 0.01 & 0.01 & 0.03 & 0.05 \\
\hline 14 & $\alpha_{g}$ & Intercept for $\operatorname{Pr}$ (gravid) & 0.01 & 0.02 & 0.02 & 0.03 \\
\hline 15 & $\beta_{\mathrm{s} 1}$ & Linear effect of size on survival & 0.09 & 0.03 & 0.00 & 0.01 \\
\hline
\end{tabular}


Table 18. Elasticity of the population growth rate, $\lambda$, of giant gartersnakes in Sacramento Valley, California, to perturbation of vital rate model parameters for the deterministic Integral Projection Model, based primarily on reproductive and survival data from Hansen and others $(2015,2017)$, for four values of $p_{\text {rec. }}$.

[All vital rate parameters were increased by 1 percent above their mean value, and the corresponding elasticity was calculated. We report the absolute value of elasticity. SVL, snout-vent length; SD, standard deviation; Pr, probability]

\begin{tabular}{lllllll}
\hline \multirow{2}{*}{ Rank } & Parameter & & \multicolumn{5}{c}{ Description } & \multicolumn{2}{c}{$p_{\text {rec }}$} \\
\cline { 4 - 7 } & & & 0.1 & $\mathbf{0 . 2}$ & $\mathbf{0 . 3}$ & $\mathbf{0 . 4}$ \\
\hline 1 & $\mathrm{~L}_{\infty}$ & Asymptotic size & 0.69 & 0.75 & 0.79 & 0.81 \\
2 & $\mu_{\mathrm{rec}}$ & Mean recruit SVL & 0.29 & 0.47 & 0.61 & 0.73 \\
3 & $\beta_{\mathrm{f}}$ & Linear effect of size on fecundity & 0.33 & 0.41 & 0.46 & 0.50 \\
4 & $\alpha_{\mathrm{f}}$ & Fecundity intercept & 0.21 & 0.28 & 0.33 & 0.36 \\
5 & $\beta_{\mathrm{s} 1}$ & Linear effect of size on survival & 0.38 & 0.34 & 0.31 & 0.30 \\
6 & $\mathrm{p}_{\mathrm{rec}}$ & Probability of recruitment & 0.19 & 0.26 & 0.30 & 0.33 \\
7 & $\mathrm{p}_{\mathrm{sb}}$ & Probability of stillbirth & 0.19 & 0.26 & 0.30 & 0.33 \\
8 & $\mathrm{p}_{\mathrm{f}}$ & Proportion of female offspring & 0.19 & 0.26 & 0.30 & 0.33 \\
9 & $\alpha_{\mathrm{s}}$ & Survival intercept & 0.29 & 0.27 & 0.26 & 0.26 \\
10 & $k$ & Von Bertalanffy growth coefficient & 0.13 & 0.18 & 0.20 & 0.22 \\
11 & $\beta_{\mathrm{g}}$ & Slope for Pr(gravid) & 0.01 & 0.01 & 0.03 & 0.05 \\
12 & $\sigma_{\mathrm{rec}}$ & SD recruit SVL & 0.01 & 0.02 & 0.02 & 0.03 \\
13 & $\alpha_{\mathrm{g}}$ & Intercept for Pr(gravid) & 0.01 & 0.02 & 0.02 & 0.03 \\
\hline
\end{tabular}

\section{Stochastic IPM}

The long-term stochastic population growth rates $\left(\lambda_{\mathrm{s}}=0.69,0.81,0.92\right.$, and 1.01 for $p_{\text {rec }}=0.1$, $0.2,0.3$, and 0.4 , respectively) from the stochastic USGS IPM are slightly lower than the corresponding growth rates from the deterministic USGS IPM $(\lambda=0.74,0.87,0.97$, and 1.06). This is expected because adding stochastic variation in vital rates will, in general, decrease the long-term population growth rate (unless there is a negative correlation between vital rates; Fieberg and Ellner, 2001). The elasticity surface for the stochastic IPM is very similar to the elasticity surface of the deterministic IPM; therefore, we can draw similar conclusions about which vital rates and sizetransitions most affect the rate of population growth. Population growth in the stochastic IPM is most affected by the survival and growth of large adult females, followed by the survival and growth of subadult individuals. The recruitment of 1-year-old snakes has a smaller effect on $\lambda_{\mathrm{s}}$ than the survival of large adult females, but the effect of the recruitment process increases as $p_{\text {rec }}$ increases (fig. 25). 

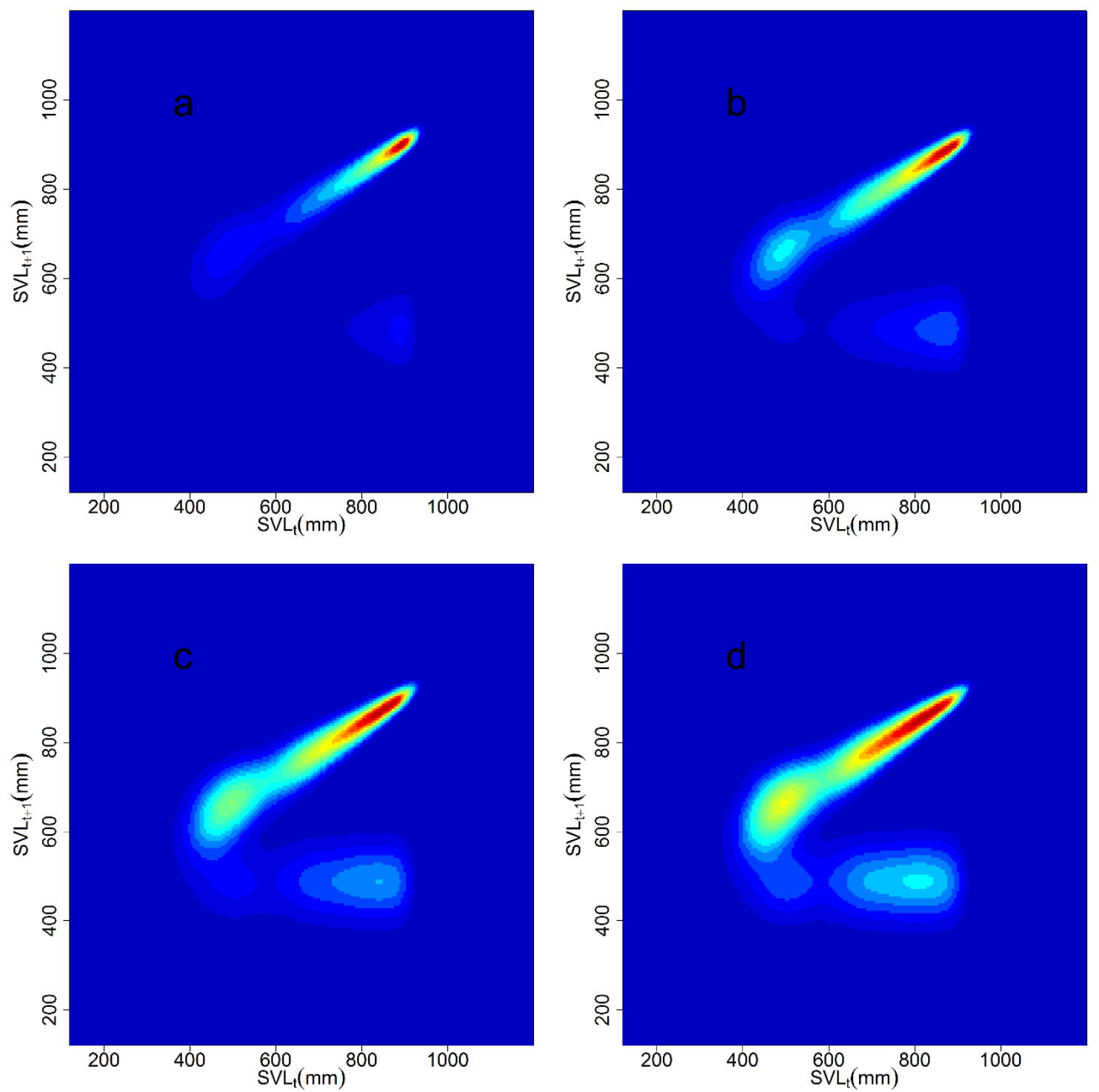

Figure 25. Elasticity surface for the stochastic U.S. Geological Survey Integral Projection Model. Panels differ in the probability of recruitment: (a) $p_{\text {rec }}=0.1$, (b) $p_{\text {rec }}=0.2$, (c) $p_{\text {rec }}=0.3$, (d) $p_{\text {rec }}=0.4$. The value of each cell on the surface represents the proportional increase in the population growth rate, $\lambda$, from increasing that sizetransition probability. The light blue shape in the lower right represents the elasticity of $\lambda$ to changes in the fecundity components of the Integral Projection Model (IPM), and the diagonal band in the center represents the elasticity of $\lambda$ to changes in the survival-growth components of the IPM. Cool colors indicate increasing the transition probability has a small effect on $\lambda$; warm colors indicate increasing the transition probability has a large effect on $\lambda$. 


\section{Discussion}

This study shows that an IPM approach is well suited to modeling the demography of giant gartersnakes because of strong evidence that growth, fecundity, probability of reproduction, and survival are all size dependent. Data on vital rates for giant gartersnakes were combined from many different populations, years, and studies to build a comprehensive model of the way in which populations of giant gartersnakes change over time. We found that the population growth rate of giant gartersnakes is most affected by the growth and survival of large adult females and the size at which recruits enter the population when they are 1 year old. We also found that the elasticity of many vital rates varied with the probability of recruitment, and therefore the population growth rate. These findings have implications for priority research questions for these important life stages. Our findings also inform conservation and management efforts targeted to have the greatest effect on the persistence and growth of this threatened species.

One potentially confusing aspect of our results is the different implications of the elasticity surfaces (for example, figs. 23, 24) and the elasticity of the underlying vital rate parameters (tables 17, 18). For example, although the "recruitment region" in the lower right of the elasticity surfaces appears to show a small effect of recruitment on population growth rate, $\lambda$, compared to the survival of adult females (figs. 23, 24), increasing the average size of recruits has a big effect on $\lambda$ when a single vital rate parameter is perturbed at a time (tables 17, 18). The elasticity surfaces in figures 23 and 24 show the effect on $\lambda$ of increasing the probability of a life-stage transition between two particular sizes in the IPM. Thus, the low elasticity in the recruitment region reflects that increasing the probability of a female of a given size on the x-axis (for example, $800 \mathrm{~mm} \mathrm{SVL}$ ) producing recruits of a single, specific size on the y-axis (for example, $450 \mathrm{~mm} \mathrm{SVL}$ ) does not greatly affect $\lambda$. The results for the mean size of recruits in tables 17 and 18 essentially show the integrated elasticity over this whole recruitment region. In other words, how much would $\lambda$ increase if we shift the whole recruitment region of the IPM higher up the $y$-axis to a larger mean size at recruitment? Increasing the population-level average of size at recruitment has a big effect because it means every female is producing larger recruits on average, and all of those recruits thus have a higher probability of survival and reaching a larger size by the time they are 2 years old. The importance of the survival of large adult females is highlighted by both the bright red region in the upper right of the elasticity surface and the elasticity of $\lambda$ to vital rate parameters. The size, survival, and fecundity of adult females all appear to be among the most important vital rates.

The importance of the survival of large adult females for giant gartersnake population growth points to potential research and management actions that could benefit this species. To design effective management actions that increase the survival rate of large adult females, there first must be an evaluation of what factors affect the survival of this life stage. Radio-telemetry studies have found that the mortality risk for adult female giant gartersnakes is much lower in terrestrial habitats, where they take refuge, than in aquatic habitats, where they forage (Halstead and others, 2012). Also, adult female giant gartersnakes tend to select micro-habitats that provide cover, such as emergent wetland vegetation (especially Schoenoplectus acutus [tules]), terrestrial vegetation, and litter (Halstead and others, 2016). These findings indicate that management actions that promote the availability of terrestrial habitat with vegetative cover near aquatic habitats will likely increase the survival of large adult female giant gartersnakes. Because adult female giant gartersnakes select emergent vegetation within wetlands and emergent vegetative cover was positively related to the survival of giant gartersnakes (section 3), promoting the establishment of emergent vegetation such as tules will also likely benefit this life stage. Hansen and others (2015) found that the survival of giant gartersnakes in the American Basin (adjacent to the Natomas Basin, immediately north) was negatively correlated to the amount of precipitation that fell from April 15-May 15 in that year. Although it is not possible to control the amount or timing of 
precipitation that falls on giant gartersnake habitats, this finding likely points to the importance of water management and rice growing practices on giant gartersnake survival. Future research into the interaction between precipitation, water management in wetland habitats (including canals associated with rice agriculture), rice growing practices, and the survival of adult female giant gartersnakes could be valuable for elucidating the conditions that promote high survival and thus population growth.

As the size of a female giant gartersnake increases, the number of offspring it can produce also increases. It is intuitive then that population growth is positively related to the asymptotic length of females. In addition, Hansen and others (2015) found a positive, linear relationship between size and survival, such that the largest individuals had the highest annual survival probability. In a population with a linear size-survival relation, the longer the asymptotic length, the higher the annual survival for the largest females in the population. Asymptotic length varies among populations of giant gartersnakes (section 1), and thus it could be worthwhile to further study how differences in population growth among sites relate to variation in asymptotic length.

According to both IPMs, population growth of giant gartersnakes is also highly affected by the size of 1-year-old snakes when they recruit to the population, especially when the probability of recruitment is high. Large 1-year-old snakes likely have a twofold effect on population growth. First, larger snakes have higher survival, so the faster that snakes reach a large size, the higher their chances of surviving to reach adulthood and contributing to the next generation. A relationship between the size of young snakes and their survival has been documented in other natricine species. In T. sirtalis fitchi (valley gartersnake) and Nerodia sipedon (northern watersnake), survival of neonate snakes was positively related to body size (Jayne and Bennett, 1990; Kissner and Weatherhead, 2005). Second, larger 1-year-old snakes are more likely to reach reproductive maturity by the time they are 2 years old, then reproduce at either 2 or 3 years of age. Reaching reproductive maturity at an earlier age might increase the average reproductive output of an individual because with a short life span and relatively low annual survival (50-60 percent), the earlier a female begins to reproduce the more reproductive bouts she can have and thus the more offspring she can produce in her lifetime. Little is known about the factors that affect the growth of young giant gartersnakes in their first year, although some evidence indicates that growth of young giant gartersnakes might be variable among populations. The growth of giant gartersnakes, in general, is positively related to the abundance of anuran prey and the total amount of precipitation in a year (section 1). Neonate giant gartersnakes appear to prefer anurans over other prey types (Ersan, 2015), although Rana catesbeiana (American bullfrogs) can also act as predators on young giant gartersnakes (Wylie and others, 2003). On the basis of the results of our elasticity analysis, the growth and survival of giant gartersnakes in their first year, and the factors that affect these processes, are important targets for future research efforts. Key questions include whether and how management interventions could affect the size of giant gartersnake recruits.

Calculating the elasticity of $\lambda$ to changes in life-stage transitions and vital rate parameters is an established method used to determine where to focus conservation and research efforts for threatened species (Caswell, 2000). For example, in a classic study, Crouse and others (1987) built a stage-based matrix population model for the threatened Caretta caretta (Loggerhead sea turtle). Crouse and others (1987) found that common conservation practices focused on protecting eggs were unlikely to be effective because of the low elasticity of this life stage. Contrary to expectations, the survival of juveniles had the highest elasticity. This finding led to the widespread implementation of "Turtle Excluder Devices," which could increase the survival rate of turtles caught in shrimp trawls, with concomitant increases in population growth rates (Crowder and others, 1994). The most powerful insight from elasticity analysis of demographic models is this: management actions that affect vital rates with high elasticity (for example, increasing the survival rate of juvenile Loggerhead sea turtles) should effect large changes in $\lambda$. Because the probability of a population going extinct is inversely proportional 
to $\lambda$ (Caswell, 2001), a management action that increases $\lambda$ will decrease the probability of a population (or species) going extinct. Not all cases may be as straightforward as the example of Loggerhead sea turtles, however. When interpreting the results of elasticity analysis in a conservation context, it is important to consider which vital rates could actually be affected by management actions (De Kroon and others, 2000). For example, imagine a scenario where elasticity analysis of a demographic model (for example, an IPM) for an endangered bird found that the parameter with the highest elasticity was the number of offspring produced by a female. If fecundity was fixed (that is, evolutionarily constrained) to a single egg per clutch in this species, it would not be possible through management action to affect this life-history parameter, and action would likely be directed elsewhere. Another scenario could be that the elasticity of population growth of an endangered Crotalus horridus (timber rattlesnake) population was highest for adult survival, but annual adult survival was already very high ( $>80$ percent, as seen in timber rattlesnakes; Brown and others, 2007). In that case, it might be difficult to increase adult survival beyond its naturally high level, especially if most mortality is not humanmediated, but rather the result of factors like climatic extremes. Thus, the ability to improve population growth of giant gartersnakes by targeting important vital rates will be constrained by the degree to which those vital rates can by affected by management interventions.

With the data available, we were able to obtain estimates of how the growth, survival, and reproduction of giant gartersnakes vary as a function of size, and how these vital rates vary over time. The probability of recruitment from neonate to 1-year-old snake the following year (that is, neonate survival) remains more uncertain. We estimated this parameter indirectly by comparing the expected reproductive output in a population in 1 year (based on the model estimated number and size of adult females present) to the estimated cohort of 1-year-old snakes the following year. To estimate the number of adult females and the number of 1-year-old snakes in the population, we used a Bayesian CMR model that accounted for size-dependent capture probability. Therefore our estimates of reproductive output from adult females and the abundance of 1-year-old snakes are based on estimates of the total number of snakes in the population, not just snakes that were captured. We also reviewed the literature on neonate survival and recruitment in gartersnakes and other natricine snakes to get a sense of a realistic value for this vital rate. Our estimates of the probability of recruitment generally ranged from 10 to 20 percent, which is substantially lower than published estimates from closely related snakes, which largely ranged from 20 to 40 percent. Whether this discrepancy indicates a true difference between giant gartersnakes and other species, or not, cannot be determined from this study. It is possible that the probability of recruitment is lower in giant gartersnakes because they largely inhabit altered habitats (for example, agricultural canals); other snake populations have been studied in less disturbed, natural habitats. It is also possible that the lower estimates are an artifact of our methods. To account for this uncertainty, we repeated our elasticity analyses for four scenarios with the probability of recruitment varying within a range of reasonable values. These four recruitment scenarios effectively represent scenarios ranging from a rapidly declining population to a rapidly growing population.

The parameters that had the greatest elasticity depended on the probability of recruitment and therefore on whether the modeled population was declining, stable, or increasing in abundance. When the probability of recruitment is low, the population is expected to decline, and the relative importance of survival is greater. This pattern makes sense because if recruitment is low, few new individuals are being added to the population, so the change in abundance from year $t$ to year $t+1$ is largely driven by how many individuals already present in the population in year $t$ survive to year $t+1$. In contrast, the elasticity analysis shows that the size of recruits (at 1 year old) and other fecundity parameters are most important for population growth when the probability of recruitment is high. When the probability of recruitment is high, the population is likely to be growing; therefore, the addition of new individuals through reproduction and recruitment makes a major contribution to the abundance of the population. 
Therefore, managers' ability to choose the most effective action for a giant gartersnake population may depend on the recent trend in abundance, and the most effective action for a population in decline may not be the most effective for a growing population.

Although its elasticity was much lower than other parameters, the probability of neonates surviving to 1 year old is clearly an important vital rate because a population can persist only if recruitment of newborn individuals is sufficient to balance out the loss of reproductive adults. The only way to directly measure the probability of recruitment for giant gartersnakes would be to mark neonates immediately after birth and recapture them in subsequent years to estimate their probability of survival in the first year. Obtaining a precise estimate of neonate survival would likely be difficult for giant gartersnakes because the low capture probability of small snakes (section 3), combined with low survival, means year over year recaptures will be low. An alternative could be to mark and release neonate snakes within some type of enclosure within their natural habitat, as was done for Nerodia sipedon (northern watersnakes) in Ontario, Canada (Kissner and Weatherhead, 2005). Constructing an enclosure that prevents neonate semi-aquatic snakes from escaping but allows for normal activities, such as foraging, may be difficult.

Most of the analysis in this study focused on IPMs built from the mean vital rates estimated for giant gartersnakes. We know that growth, survival, and reproduction all vary among years for giant gartersnakes, however. Stochastic annual variation in vital rates is widely observed in natural populations, and consequently, theoreticians have developed methods to create stochastic IPMs where the vital rates fluctuate from year to year to better reflect real population dynamics (Rees and Ellner, 2009; Metcalf and others, 2015). The inferences from the deterministic and stochastic IPMs in this study were very similar because elasticity analysis from both models showed the importance of the survival of large adult females for population growth. One difference between the stochastic and deterministic IPMs is that the projected rate of population growth was lower in the stochastic IPM, where growth, reproduction, and survival parameters varied among years. This finding was expected, based on theoretical studies of population growth in the face of stochasticity-increasing temporal variability leads to a smaller population growth rate and higher probability of extinction (Lande and Orzack, 1988). Thus efforts to project the growth and probability of persistence or extinction of a giant gartersnake population using an IPM in the future ideally would include temporal variability in vital rates to avoid overestimating growth and underestimating the probability of extinction. Our stochastic IPM could be used to simulate the effect of proposed management actions or climate variables on the growth rate and probability of persistence of giant gartersnake populations. The reliability and usefulness of such simulations will depend on understanding the relationships between potential management actions, or future climate, and expected changes in growth, survival, and fecundity.

Our study helps address how the results of the vital rate models, IPM, and elasticity analysis can best inform conservation planning and management for giant gartersnakes. Specifically, the IPM, and the results of our elasticity analysis, could be used as a decision-support toolset that integrates many different sources of data on giant gartersnake population biology; clearly states its structure, assumptions, and uncertainties; and highlights what is known and unknown about the demography of this threatened species (Burgman and others, 2000). In addition to the elasticity analysis performed in this study, future studies could use our stochastic IPM to simulate the effect of management scenarios on the growth and probability of persistence (or conversely, extinction probability) of a population as part of a demographic PVA. PVAs are a widely used method for quantifying the risk of extinction for endangered species and are best used for short-term projections that compare the relative risk of extinction under different management scenarios (Beissinger and Westphal, 1998). Indeed, the recovery plan for the giant gartersnake calls for conducting a PVA as one of the key recovery actions for the species (Recovery action 7.4 of the Giant Gartersnake Recovery Plan, U.S. Fish and Wildlife Service, 
2017). The stochastic IPM could be used for any future PVA exercise for giant gartersnakes, instead of the deterministic IPM, because our results have shown that the key vital rates of survival, individual growth, and fecundity vary over time (sections $1-3$ ). This temporal variation in vital rates means that the long-term stochastic population growth rate, $\lambda_{s}$, will give a more realistic estimate of the probability of a population going extinct in the future than the value of $\lambda$ calculated from a deterministic IPM, which ignores temporal variation in vital rates; the deterministic IPM can overestimate population growth (Ellner and others, 2016).

The success of any application of our IPM to the conservation and management of giant gartersnakes hinges upon understanding relationships between different potential management actions and specific vital rates. For example, our elasticity analysis showed that the survival of large adult females is important for population growth, but translating that finding to inform improved conservation and management requires strong links between actions (for example, improving habitat quality through the establishment of emergent vegetation or the amount of flooded habitat on the landscape) and the desired response (increased survival). The growth rate of giant gartersnake populations was also found to be highly sensitive to the size of 1-year-old snakes, but unfortunately there is no information on why that life-history trait might vary among populations or how management actions could increase the growth of giant gartersnakes during their first year. Therefore, now that we have a general model for the demography of giant gartersnakes, the largest information gap is why key vital rates and life-history parameters vary over space and time and which actions would be most likely to positively affect those rates and parameters to increase population growth.

Since its listing as state and federally threatened species, a wealth of data has been collected on the survival, growth, and reproduction of giant gartersnakes. The population model developed in this study integrates these data and provides novel insights into the demography of giant gartersnakes. Our primary conclusions are that the survival of large adult female gartersnakes and the size at which 1-yearold snakes recruit to the population greatly affect the growth rate of giant gartersnake populations. Although the study provides much new information on the demography of giant gartersnakes, the development of a demographic population projection model is just a first step. Our demographic model is a building block that can be expanded and applied in many ways to inform land and resource managers planning for this threatened species. We look forward to seeing how other researchers, managers, and conservation groups use, revise, and build upon the work presented here.

\section{Summary and Conclusions}

A study was conducted by the U.S. Geological Survey, in cooperation with the California Department of Water Resources, to develop a demographic population model for the threatened giant gartersnake in the Sacramento Valley, California. This study integrated data on the growth, reproduction, and survival of giant gartersnakes collected from several populations over a period 
spanning 22 years. The results of this study provide vital information on the population biology of this threatened species, and could be used to target important research, conservation, and management actions in the future. The findings are summarized below.

- Giant gartersnakes exhibit indeterminate growth that is well described by a von Bertalanffy growth function. Growth is initially rapid, but growth slows with age as individuals approach an asymptotic or maximum length after reaching reproductive maturity.

- Giant gartersnake growth is positively related to the amount of precipitation at a site in a year.

- There is an interactive effect of frog abundance and fish abundance on giant gartersnake growth. Giant gartersnake growth is positively related to frog abundance when fish abundance is low, and giant gartersnake growth is negatively related to fish abundance when frog abundance is high.

- Both the fecundity (litter size) and probability of reproduction of female giant gartersnakes increase with snout-vent length (SVL).

- Survival increases with SVL in giant gartersnakes. The data analyzed in this study showed that the annual survival rate of giant gartersnakes increased with body length up to a peak, then declined slightly for the largest individuals.

- Survival of giant gartersnakes varied greatly over time and among populations.

- Survival of giant gartersnakes was positively related to the amount of emergent vegetation cover and the abundance of fish at a site.

- The survival of neonate giant gartersnakes during their first year (that is, the probability of recruitment) is largely unknown, and we relied on indirect estimation methods and published values for closely related species to generate reasonable values for this important parameter.

- Elasticity analysis of the giant gartersnake Integral Projection Model (IPM) revealed that population growth is most affected by the growth and survival of large adult females and by the size at which 1-year-old snakes recruit to the population.

- The relative effect of survival in relation to growth and fecundity on population growth varied with the probability of recruitment and therefore whether the population was declining or increasing. Elasticity was higher for survival when recruitment was low and the population would be expected to decline. Elasticity was higher for growth and fecundity when recruitment was high and the population would be expected to increase.

- IPMs constructed from different datasets that include fecundity and survival of giant gartersnakes had similar elasticity patterns and produced similar conclusions about the vital rates and life-stage transitions that most affect population growth.

- Elasticity analysis of deterministic and stochastic IPMs produced similar conclusions about the importance of different vital rates and life-stage transitions to population growth of giant gartersnakes.

- On the basis of the results of the elasticity analysis in this study, future research efforts are best focused on factors that affect the survival of large adult females and the size of 1-year-old snakes.

- The population models developed in this study can be expanded and revised as new data are collected and analyzed on the growth, reproduction, and survival of giant gartersnakes.

- The population models developed in this study, the stochastic IPM in particular, could be applied in the future to a Population Viability Analysis. Population Viability Analysis could be used to simulate the predicted effects of management and conservation actions on the growth and persistence of giant gartersnake populations. 


\section{References Cited}

Adams, M.J., 2000, Pond permanence and the effects of exotic vertebrates on anurans:

Ecological Applications, v. 10, p.559-568.

Aldridge, R.D., Flanagan, W.P., and Swarthout, J.T., 1995, Reproductive biology of the water snake Nerodia rhombifer from Veracruz, Mexico, with comparisons of tropical and temperate snakes: Herpetologica, v. 51, p. 182-192.

Altwegg, R., Dummermuth, S., Anholt, B.R., and Flatt, T., 2005, Winter weather affects asp viper Vipera aspis population dynamics through susceptible juveniles: Oikos, v. 110, p. 55-66.

Andrews, R.M., 1982, Patterns of growth in reptiles, in Gans, C., and Pough, F.H., eds., Biology of the Reptilia: New York, Academic Press, v.13, p. 273-320.

Autumn, K., and De Nardo, D.F., 1995, Behavioral thermoregulation increases growth rate in a nocturnal lizard: Journal of Herpetology, v. 29, p.157-162.

Batzer, D.P., and Wissinger, S.A., 1996, Ecology of insect communities in nontidal wetlands: Annual Review of Entomology, v. 41, p. 75-100.

Beissinger, S., and Westphal, M., 1998, On the use of demographic models of population viability in endangered species management: The Journal of Wildlife Management, v. 62, p. 821-841.

Benton, T., and Grant, A., 1999, Elasticity analysis as an important tool in evolutionary and population ecology: Trends in Ecology \& Evolution, v. 14, p. 467-471.

Bernardo, J., 1993, Determinants of maturation in animals: Trends in Ecology and Evolution, v. 8, p. 166-173.

Bjorndal, K.A., Bolten, A.B., and Chaloupka, M.Y., 2000. Green turtle somatic growth model: evidence for density dependence: Ecological Applications, v. 10, p. 269-282.

Blums, P., Nichols, J.D., Hines, J.E., Lindberg, M.S., and Mednis. A., 2005, Individual quality, survival variation and patterns of phenotypic selection on body condition and timing of nesting in birds: Oecologia, v. 143, p. 365-376.

Bonner, S. J., Morgan, B.J.T., and King, R., 2010, Continuous covariates in mark-recapturerecovery analysis: a comparison of methods: Biometrics, v. 66, p. 1256-1265.

Bronikowski, A.M., and Arnold, S.J., 1999, The evolutionary ecology of life history variation in the garter snake Thamnophis elegans: Ecology, v. 80, p. 2314-2325.

Brook, B.W., Sodhi, N.S., and Bradshaw, C.J.A., 2008, Synergies among extinction drivers under global change: Trends in Ecology and Evolution, v. 23, p. 453-460.

Brooks, S. P., and Gelman, A., 1998, General methods for monitoring convergence of iterative simulations: Journal of Computational and Graphical Statistics, v. 7, p. 434-455.

Brown, W. S., Kéry, M., and Hines, J.E., 2007, Survival of Timber Rattlesnakes (Crotalus horridus) estimated by capture-recapture models in relation to age, sex, color morph, time, and birthplace: Copeia, v. 3, p. 656-671.

Brown, G. P., and Weatherhead, P.J., 1999a, Growth and sexual size dimorphism in northern water snakes (Nerodia sipedon): Copeia, v. 1999, p. 723-732.

Brown, G. P., and Weatherhead, P.J., 1999b, Demography and sexual size dimorphism in northern water snakes, Nerodia sipedon: Canadian Journal of Zoology, v. 77, p. 1358-1366.

Burgman, M., Possingham, H., Young, A.G., and Clarke, G.M., 2000, Population viability analysis for conservation: the good, the bad, and the undescribed, in Young, A.G., and Clarke, G.M., eds.. Genetics, demography and viability of fragmented populations: London, Cambridge University Press, p. 97-112. 
California Department of Fish and Game Commission, 1971, California Code of Regulations: Animals of California Declared to be Endangered or Threatened: California Department of Fish and Game Commission, Title 14, section 670.5.

Carpenter, B., Gelman, A., Hoffman, M., Lee, D., Goodrich, B., Betancourt, M., Brubaker, M.A., Li, P., and Riddell, A., 2017, Stan: A Probabilistic Programming Language: Journal of Statistical Software, v. 76, p. 1-32.

Carpenter, C. C., 1952, Growth and maturity of the three species of Thamnophis in Michigan: Copeia, v. 1952, no. 4, p. 237-243.

Casazza, M. L., Wylie, G.D., and Gregory, C.J., 2000, A funnel trap modification for surface collection of aquatic amphibians and reptiles: Herpetological Review, v. 31, p. 91-92.

Caswell, H., 2000, Prospective and retrospective perturbation analyses: their roles in conservation biology: Ecology, v. 81, no. 619-627.

Caswell, H., 2001, Matrix population models (2d ed.) Sunderland, Mass., Sinauer Associates, $713 \mathrm{p}$.

Chabreck, R. H., and Joanen, T., 1979, Growth rates of American alligators in Louisiana: Herpetologica, v. 35, p. 51-57.

Coates, P.S., Wylie, G.D., Halstead, B.J., and Casazza, M.L., 2009, Using time-dependent models to investigate body condition and growth rate of the giant gartersnake: Journal of Zoology, v. 279, p. 285-293.

Coop, L.B., 2010, U.S. degree-day mapping calculator, Version 4.0, accessed October, 11, 2016 at http://uspest.org/cgi-bin/usmapmaker.pl.

Cormack, R.M., 1964, Estimates of survival from the sighting of marked animals: Biometrika, v. 51, p. 429-438.

Coulson, T., 2012, Integral projections models, their construction and use in posing hypotheses in ecology: Oikos, v. 121, p. 1337-1350.

Crouse, D., Crowder, L., and Caswell, H., 1987, A stage-based population model for loggerhead sea turtles and implications for conservation: Ecology, v. 68, p. 1412-1423.

Crowder, L.B., Crouse, D.T., Heppell, S.S., and Martin, T.H., 1994, Predicting the impact of turtle excluder devices on loggerhead sea turtle populations: Ecological Applications, v. 4, no. 3, p. 437-445.

Dahlgren, J.P., García, M.B., and Ehrlén, J., 2011, Nonlinear relationships between vital rates and state variables in demographic models: Ecology, v. 92, p. 1181-1187.

De Kroon, H., Van Groenendael, J., and Ehrlén, J., 2000, Elasticities: a review of methods and model limitations: Ecology, v. 81, p. 607-618.

Denwood, M.J., 2015, runjags: An R package providing interface utilities, model templates, parallel computing methods and additional distributions for MCMC models in JAGS: Journal of Statistical Software, v. 2, p. 16-48.

Diaz, H.F., and Wahl, E.R., 2015, Recent California water year precipitation deficits: A 440-year perspective: Journal of Climate, v. 28, p. 4637-4652.

Dirzo, R., Young, H.S., Galetti, M., Ceballos, G., Isaac, N.J.B., and Collen, B., 2014, Defaunation in the Anthropocene: Science, v. 345, p. 401-406.

Doak, D.F., Morris, W.F., Pfister, C., Kendall, B.E., and Bruna, E.M., 2005, Correctly estimating how environmental stochasticity influences fitness and population growth: The American Naturalist, v. 166, p. E14-21.

Doak, D., Kareiva, P., and Klepetka, B., 1994, Modeling oopulation viability for the Desert Tortoise in the Western Mojave Desert: Ecological Applications, v. 4, p. 446-460. 
Dunham, A.E., 1978, Food availability as a proximate factor influencing individual growth rates in the Iguanid lizard Sceloporus merriami: Ecology, v. 59, p. 770-778.

Easterling, M.R., Ellner, S.P., and Dixon, P.M., 2000, Size-specific sensitivity: applying a new structured population model: Ecology, v. 81, p. 694-708.

Eaton, M.J., and Link, W.A., 2011, Estimating age from recapture data: Integrating incremental growth measures with ancillary data to infer age-at-length: Ecological Applications, v. 21, p. 2487-2497.

Elderd, B.D., and Miller, T.E.X., 2016, Quantifying demographic uncertainty: Bayesian methods for integral projection models: Ecological Monographs, v. 86, p. 125-144.

Ellner, S.P., Childs, D.Z., and Rees, M., 2016, Data driven modelling of structured populations: New York, Springer, 329 p.

Enright, N.J., Franco, M., and Silvertown, J., 1995, Comparing plant life histories using elasticity analysis: the importance of life span and the number of life-cycle stages: Oecologia, v. 104, p. 79-84.

Ergon, T., and Gardner, B., 2014, Separating mortality and emigration: Modelling space use, dispersal and survival with robust-design spatial capture-recapture data: Methods in Ecology and Evolution. v. 5, p. 1327-1336.

Ersan, J.S.M., 2015, Diet and prey preference of Giant Gartersnakes (Thamnophis gigas) in the Sacramento Valley of California: Hayward, California State University_East Bay, M.S. thesis, $76 \mathrm{p}$.

Fabens, A.J., 1965, Properties and fitting of the von Bertalanffy growth curve: Growth, v. 29, p. 265-289.

Fieberg, J., and Ellner, S.P., 2001, Stochastic matrix models for conservation and management: a comparative review of methods: Ecology Letters, v. 4, p. 244-266.

Fitch, H.S., 1965, An ecological study of the garter snake, Thamnophis sirtalis: Lawrence, Kansas, University of Kansas Publications Museum of Natural History, v. 15, p. 493-564.

Ford, N.B., and Karges, J.P., 1987, Reproduction in the checkered garter snake, Thamnophis marcianus, from southern Texas and northeastern Mexico: seasonality and evidence for multiple clutches: Southwestern Naturalist, v. 32, p. 93-101.

Ford, N.B., and Killebrew, D.W., 1983, Reproductive tactics and female body size in Butler's garter snake, Thamnophis butleri: Journal of Herpetology, v. 17, p. 271-275.

Gaillard, J.-M., and Yoccoz, N.G., 2003, Temporal variation in survival of mammals: a case of environmental canalization?: Ecology, v. 84, p. 3294-3306.

Gelman, A., 2006, Prior distribution for variance parameters in hierarchical models: Bayesian Analysis, v. 1, p. 515-533.

Gibbons, J.W., and Dorcas, M.E., 2004, North American watersnakes: a natural history: Norman, Okla., University of Oklahoma Press, 496 p.

González, E.J., Rees, M., and Martorell, C., 2013, Identifying the demographic processes relevant for species conservation in human-impacted areas: Does the model matter?: Oecologia, v. 171, p. 347-356.

Gregory, P.T., and Larsen, K.W., 1993, Geographic variation in reproductive characteristics among Canadian populations of the common garter snake (Thamnophis sirtalis): Copeia, v. 1993, p. 946-958.

Gregory, P.T., Larsen, K.W., and Farr, D.R., 1992, Snake litter size = live young + dead young + yolks: Herpetological Journal, v. 2, p. 145-146. 
Halliday, T., and Verrell, P., 1988, Body size and age in amphibians and reptiles: Journal of Herpetology, v. 22, p. 253-265.

Halstead, B.J., Skalos, S.M., Wylie, G.D., and Casazza, M.L., 2015, Terrestrial ecology of semiaquatic giant gartersnakes (Thamnophis gigas): Herpetological Conservation and Biology, v. 10, p. 633-644.

Halstead, B.J., Valcarcel, P., Wylie, G.D., Coates, P.S., Casazza, M.L., and Rosenberg, D.K., 2016, Active season microhabitat and vegetation selection by Giant Gartersnakes associated with a restored marsh in California: Journal of Fish and Wildlife Management, v. 7, p. 397407.

Halstead, B. J., Wylie, G.D., Amarello, M., Smith, J.J., Thompson, M.E., Routman, E.J., and Casazza, M.L., 2011a, Demography of the San Francisco Gartersnake in coastal San Mateo County, California: Journal of Fish and Wildlife Management, v. 2, p. 41-48.

Halstead, B.J., Wylie, G.D., and Casazza, M.L., 2010, Habitat suitability and conservation of the giant gartersnake (Thamnophis gigas) in the Sacramento Valley of California: Copeia, v. 2010, p. 591-599.

Halstead, B.J., Wylie, G.D., and Casazza, M.L., 2013, Efficacy of trap modifications for increasing capture rates of aquatic snakes in floating aquatic funnel traps: Herpetological Conservation and Biology, v. 8, no. 65-74.

Halstead, B.J., Wylie, G.D., and Casazza, M.L., 2014, Ghost of habitat past: historic habitat affects the contemporary distribution of giant garter snakes in a modified landscape: Animal Conservation, v. 17, p. 144-153.

Halstead, B. J., Wylie, G.D., Casazza, M.L., and Coates, P.S., 2011b, Temporal and maternal effects on reproductive ecology of the giant gartersnake (Thamnophis gigas): The Southwestern Naturalist, v. 56, p. 29-34.

Halstead, B.J., Wylie, G.D., Coates, P.S., Valcarcel, P., and Casazza, M.L., 2012, Bayesian shared frailty models for regional inference about wildlife survival: Animal Conservation, v. 15 , p. 117-124.

Hansen, R.W., and Hansen, G.E., 1990, Thamnophis gigas (Giant Garter Snake). Reproduction: Herpetological Review, v. 21, p. 93-94.

Hansen, E.C., Scherer, R.D., White, G.C., Dickson, B.G., and Fleishman, E., 2015, Estimates of survival probability from two populations of giant gartersnakes in California's Great Central Valley: Copeia, v. 103, p. 1026-1036.

Hansen, E.C., Scherer, R.D., and Joseph, M.B., 2017, Giant gartersnake (Thamnophis gigas) growth and fecundity summary: Report to California Department of Water Resources.

Hayhoe, K., Cayan, D., Field, C.B., Frumhoff, P.C., Maurer, E.P., Miller, N.L., Moser, S.C., Schneider, S.H., Cahill, K.N., Cleland, E.E., Dale, L., Drapek, R., Hanemann, R.M., Kalkstein, L.S., Lenihan, J., Lunch, C.K., Neilson, R.P., Sheridan, S.C., and Verville, J.H., 2004, Emissions pathways, climate change, and impacts on California: Proceedings of the National Academy of Sciences of the United States of America, v. 101, p. 12422-12427.

Hobbs, N.T., and Hooten, M.B., 2015, Bayesian Models: a statistical primer for ecologists:

Princeton, N.J., Princeton University Press, 320 p.

Hooten, M.B., and Hobbs, N.T., 2015, A guide to Bayesian model selection for ecologists: Ecological Monographs, v. 85, p. 3-28.

Huber, P.R., Greco, S.E., and Thorne, J.H., 2010, Boundaries make a difference: the effects of spatial and temporal parameters on conservation planning: The Professional Geographer, v. 62, p. 409-425. 
Hyslop, N.L., Stevenson, D.J., Macey, J.N., Carlile, L.D., Jenkins, C.L., Hostetler, J.A., and Oli, M.K., 2012, Survival and population growth of a long-lived threatened snake species, Drymarchon couperi (Eastern Indigo Snake): Population Ecology, v. 54, p. 145-156.

Jayne, B.C., and Bennett, A.F., 1990, Selection on locomotor performance capacity in a natural population of garter snakes: Evolution, v. 44, p. 1204-1229.

Jensen, J.B., Bailey, M.A., Blankenship, E.L., and Camp, C.D., 2003, The relationship between breeding by the gopher frog, Rana capito (Amphibia: Ranidae) and rainfall: The American Midland Naturalist, v. 150, p. 185-190.

Jolly, G.M., 1965, Explicit estimates from capture-recapture data with both death and immigration-stochastic model: Biometrika, v. 52, p. 225-247.

Jongejans, E., Shea, K., Skarpaas, O., Kelly, D., and Ellner, S.P., 2011, Importance of individual and environmental variation for invasive species spread: a spatial integral projection model: Ecology, v. 92, p. 86-97.

Kendall, W.L., Nichols, J.D., and Hines, J.E., 1997, Estimating temporary emigration using capture-recapture data with Pollock's robust design: Ecology, v. 78, p. 563-578.

Kéry, M., and Schaub, M., 2012, Bayesian population analysis using WinBUGS: a hierarchical perspective: Burlington, Mass., Academic Press, 554 p.

King, R.B., 1986, Population ecology of the Lake Erie water snake, Nerodia sipedon insularum: Copeia , v. 1986, p. 757-772.

King, R.B., 1993, Determinants of offspring number and size in the brown snake, Storeria dekayi: Journal of Herpetology, v. 27, p. 175-185.

King, R.B., Ray, J.M., and Stanford, K.M., 2006, Gorging on gobies: beneficial effects of alien prey on a threatened vertebrate: Canadian Journal of Zoology v. 84, p. 108-115.

King, R.B., Stanford, K.M., Jones, P.C., and Bekker, K., 2016, Size matters: individual variation in ectotherm growth and asymptotic size: Plos One, v. 11, p. e0146299.

Kissner, K.J., and Weatherhead, P.J., 2005, Phenotypic effects on survival of neonatal northern watersnakes Nerodia sipedon: Journal of Animal Ecology, v. 74, p. 259-265.

Kuo, L., and Mallick, B., 1998, Variable selection for regression models: Sankhya Series B, v. 60 , p. $65-81$.

Lande, R., and Orzack, S.H., 1988, Extinction dynamics of age-structured populations in a fluctuating environment: Proceedings of the National Academy of Sciences of the United States of America, v. 85, p. 7418-7421.

Larsen, K.W., Gregory, P.T., and Antoniak, R., 1993, Reproductive ecology of the common garter snake Thamnophis sirtalis at the northern limit of its range: The American Midland Naturalist, v. 129, p. 336-345.

Lebreton, J.D., Burnham, K.P., and Clobert, J., 1992, Modeling survival and testing biological hypotheses using marked animals: a unified approach with case studies: Ecological Monographs, v. 62, p. 67-118.

Lefkovitch, L.P., 1965, The study of population growth in organisms grouped by stages:

Biometrics, v. 21, p. 1-18.

Leslie, P.H., 1945, On the use of matrices in certain population mathematics: Biometrika, v. 33, p. 183-212.

Lind, A.J., Welsh, H.H., and Tallmon, D.A., 2005, Garter snake population dynamics from a 16year study: Considerations for ecological monitoring: Ecological Applications, v. 15, p. 294303. 
Lindell, L.E., and Forsman, A., 1996, Density effects and snake predation: prey limitation and reduced growth rate of adders at high density of conspecifics: Canadian Journal of Zoology, v. 74, p. 1000-1007.

Luiselli, L., 2005, Snakes don't shrink, but "shrinkage" is an almost inevitable outcome of measurement error by the experimenters: Oikos, v. 110, p. 199-202.

Madsen, T., 1988, Reproductive success, mortality and sexual size dimorphism in the adder, Vipera berus: Ecography, v. 11, p. 77-80.

Madsen, T., and Shine, R., 1993, Phenotypic plasticity in body sizes and sexual size dimorphism in European Grass Snakes: Evolution, v. 47, p. 321-325.

Madsen, T., and Shine, 2000, R., Silver spoons and snake body sizes: prey availability early in life influences long-term growth rates of free-ranging pythons: Journal of Animal Ecology, v. 69, p. 952-958.

Madsen, T., and Shine, R., 2001, Do snakes shrink?: Oikos, v. 92, p. 187-188.

Madsen, T., Stille, B., and Shine, R., 1996, Inbreeding depression in an isolated population of adders Vipera berus: Biological Conservation, v. 75, p. 113-118.

Martin, T.G., Wintle, B.A., Rhodes, J.R., Kuhnert, P.M., Field, S.A., Low-Choy, S.J., Tyre, A.J., and Possingham, H.P., 2005, Zero tolerance ecology: Improving ecological inference by modelling the source of zero observations: Ecology Letters, v. 8, p. 1235-1246.

Merow, C., Dahlgren, J.P., Metcalf, C.J.E., Childs, D.Z., Evans, M.E.K., Jongejans, E., Record, S., Rees, M., Salguero-Gómez, R., and McMahon, S.M., 2014, Advancing population ecology with integral projection models: a practical guide: Methods in Ecology and Evolution, v. 5, p. 99-110.

Metcalf, C.J.E., Ellner, S.P., Childs, D.Z., Salguero-Gómez, R., Merow, C., McMahon, S.M., Jongejans, E., and Rees, M., 2015, Statistical modelling of annual variation for inference on stochastic population dynamics using Integral Projection Models: Methods in Ecology and Evolution, v. 6, p. 1007-1017.

Miller, D.A., Clark, W.R., Arnold, S.J., and Bronikowski, A.M., 2011, Stochastic population dynamics in populations of western terrestrial garter snakes with divergent life histories:

Ecology, v. 92, p. 1658-1671.

Mills, L.S., 2007, Conservation of wildlife populations: demography, genetics, and management: Somerset, N.J., John Wiley \& Sons, 342 p.

Mills, L.S., Doak, D.F., and Wisdom, M.J., 1999, Reliability of conservation actions based on elasticity analysis of matrix models: Conservation Biology, v. 13, p. 815-829.

Moloney, K.A., 1986, A generalized algorithm for determining category size: Oecologia, v. 69, p. 176-180.

Morris, W.F., and Doak, D.F., 2002, Quantitative conservation biology. Theory and practice of population viability analysis: Sunderland, Mass., Sinauer Associates Inc., 480 p.

Papadatou, E., Pradel, R., Schaub, M., Dolch, D., Geiger, H., Ibañez, C., Kerth, G., PopaLisseanu, A., Schorcht, W., Teubner, J., and Gimenez, O., 2012, Comparing survival among species with imperfect detection using multilevel analysis of mark-recapture data: a case study on bats: Ecography, v. 35, p.153-161.

Parker, W.S., and Plummer, M.V., 1987, Population ecology, in Seigel, R.A., Collins, J.T., and Novak, S.S., eds., Snakes: Ecology and Evolutionary Biology: Caldwell, N.J., Blackburn Press, p. 253-301

Pike, D.A., Pizzatto, L., Pike, B.A., and Shine, R., 2008, Estimating survival rates of uncatchable animals: the myth of high juvenile mortality in reptiles: Ecology, v. 89, p. 607-611. 
Plummer, M., 2003, JAGS: A program for analysis of Bayesian graphical models using Gibbs sampling: Proceedings of the 3rd International Workshop on Distributed Statistical Computing (DSC 2003), p. 20-22.

Pollock, K.H., Nichols, J.D., Brownie, C., and Hines, J.E., 1990, Statistical inference for capturerecapture experiments: Wildlife Monographs, v. 107, p. 3-97.

Powell, L.A., Conroy, M.J., Hines, J.E., Nichols, J.D., and Krementz, D.G., 2000, Simultaneous use of mark-recapture and radiotelemtry to estimate surival, movement, and capture rates: Journal of Wildlife Management, v. 64, p. 302-313.

PRISM Climate Group, 2016, PRISM Climate Data: Oregon State University, PRISM Climate Group, accessed September, 5, 2016, at http://prism.oregonstate.edu.

Pruess, K.P., 1983, Day-degree methods for pest management: Environmental Entomology, v. 12, p. 613-619.

R Core Team, 2016, R: a language and environment for statistical computing: Vienna, Austria, $\mathrm{R}$ Foundation for Statistical Computing.

Ramula, S., Rees, M., and Buckley, Y.M., 2009, Integral projection models perform better for small demographic data sets than matrix population models: a case study of two perennial herbs: Journal of Applied Ecology, v. 46, p. 1048-1053.

Rankin, R.W., Nicholson, K.E., Allen, S.J., Krützen, M., Bejder, L., and Pollock, K.H., 2016, A full-capture Hierarchical Bayesian model of Pollock's Closed Robust Design and application to dolphins: Frontiers in Marine Science, v. 3, p. 1-18.

Rees, M., and Ellner, S.P., 2009, Integral projection models for populations in temporally varying environments: Ecological Monographs, v. 79, p. 575-594.

Rees, M., and Rose, K.E., 2002, Evolution of flowering strategies in Oenothera glazioviana: an integral projection model approach: Proceedings of the Royal Society B: Biological Sciences, v. 269, p. 1509-1515.

Robeson, S. M., 2015, Revisiting the recent California drought as an extreme value: Geophysical Research Letters, v. 42, p. 6771-6779.

Rose, J.P., and Todd, B.D., 2017, Demographic effects of prolonged drought on a nascent introduction of a semi-aquatic snake: Biological Invasions, v. 19, p. 2885-2898.

Rossman, D.A., Ford, N.B., and Seigel, R.A., 1996, The Garter Snakes: Evolution and Ecology: Norman, Okla., University of Oklahoma Press, 332p.

Row, J.R., Blouin-Demers, G., and Weatherhead, P.J., 2007, Demographic effects of road mortality in black ratsnakes (Elaphe obsoleta): Biological Conservation, v. 137, p. 117-124.

Royle, J.A., Chandler, R.B., Sollman, R., and Gardner, B., 2014, Spatial Capture-Recapture: Amsterdam, Elsevier, 612 p.

Schofield, M.R., and Barker, R.J., 2011, Full open population capture-recapture models with individual covariates: Journal of Agricultural, Biological, and Environmental Statistics, v. 16, p. 253-268.

Sebens, K.P., 1987, The ecology of indeterminate growth in animals: Annual Review of Ecology and Systematics, v. 18, p. 371-407.

Seber, G.A.F., 1965, A note on the multiple-recapture census: Biometrika, v. 52, p. 249-259.

Seigel, R.A., and Fitch, H.S., 1985, Annual variation in reproduction in snakes in a fluctuating environment: Journal of Animal Ecology, v. 54, p. 497-505.

Shine, R., 1991, Why do larger snakes eat larger prey items?: Functional Ecology, v. 5, p. 493502.

Shine, R., 1994, Sexual size dimorphism in snakes revisited: Copeia, v. 1994, p. 326-346. 
Shine, R., and Charnov, E.L., 1992, Patterns of survival, growth, and maturation in snakes and lizards: American Naturalist, v. 139, p. 1257-1269.

Sparkman, A.M., Arnold, S.J., and Bronikowski, A.M., 2007, An empirical test of evolutionary theories for reproductive senescence and reproductive effort in the garter snake Thamnophis elegans: Proceedings of the Royal Society B: Biological Sciences, v. 274, p. 943-950.

Sparkman, A.M., Bronikowski, A.M., Billings, J.G., Von Borstel, D., and Arnold, S.J., 2013, Avian predation and the evolution of life histories in the garter snake Thamnophis elegans: The American Midland Naturalist, v. 170, p. 66-85.

Stanford, K., and King, R., 2004, Growth, survival, and reproduction in a northern Illinois population of the plains gartersnake, Thamnophis radix: Copeia, v. 2004, p. 465-478.

Stewart, J. R., Blackburn, D.G., Baxter, D.C., and Hoffman, L.H., 1990, Nutritional provision to embryos in a predominantly lecithotrophic placental reptile, Thamnophis ordinoides (Squamata: Serpentes): Physiological Zoology, v. 63, p. 722-734.

Su, Y.-S., and Yajima, M., 2015. R2jags: Using R to run "JAGS": The Comprehensive R Network Web site, accessed July 18, 2016, at https://cran.rproject.org/web/packages/R2jags/index.html

Thomson, D.L., Cooch, E.G., and Conroy, M.J., 2009, Modeling demographic processes in marked populations: New York, Springer, $1132 \mathrm{p}$.

Todd, B.D., and Rothermel, B.B., 2006, Assessing quality of clearcut habitats for amphibians: Effects on abundances versus vital rates in the southern toad (Bufo terrestris): Biological Conservation, v. 133, p. 178-185.

Todd, B.D., and Winne, C.T., 2006, Ontogenetic and interspecific variation in timing of movement and responses to climatic factors during migrations by pond-breeding amphibians: Canadian Journal of Zoology, v. 84, p. 715-722.

Tuttle, K.N., and Gregory, P.T., 2012, Growth and maturity of a terrestrial ectotherm near its northern distributional limit: does latitude matter?: Canadian Journal of Zoology, v. 90, p. $758-765$.

U.S. Fish and Wildlife Service, 1993, Endangered and threatened wildlife and plants: determination of threatened status for the giant garter snake: Federal Register, v. 58, p. 5405354066.

U.S. Fish and Wildlife Service, 2017, Recovery plan for the Giant Garter Snake, Thamnophis gigas: Sacramento, Calif., U.S. Fish and Wildlife Service, 78 p.

Wallace, K., Leslie, A., and Coulson, T., 2013, Re-evaluating the effect of harvesting regimes on Nile crocodiles using an integral projection model: Journal of Animal Ecology, v. 82, p. 155165.

Waye, H.L., and Gregory, P.T., 1998, Determining the age of garter snakes (Thamnophis spp.) by means of skeletochronology: Canadian Journal of Zoology, v. 76, p. 288-294.

Weatherhead, P.J., and Blouin-Demers, G., 2004, Long-term effects of radiotelemetry on black ratsnakes: Wildlife Society Bulletin, v. 32, p. 900-906.

Weatherhead, P.J., Brown, G.P., Prosser, M.R., and Kissner, K.J., 1998, Variation in offspring sex ratios in the northern water snake (Nerodia sipedon): Canadian Journal of Zoology, v. 76, p. 2200-2206.

Weatherhead, P.J., Brown, G.P., Prosser, M.R., and Kissner, K.J., 1999, Factors affecting neonate size variation in northern water snakes, Nerodia sipedon: Journal of Herpetology, v. 33 , p. 577-589. 
White, G.C., and Burnham, K.P., 1999, Program MARK: Survival estimation from populations of marked animals: Bird Study, v. 46, p. 120-138.

Williams, B.K., Nichols, J.D., and Conroy, M.J., 2002, Analysis and management of animal populations: San Diego, Calif., Academic Press, 817 p.

Winne, C.T., Willson, J.D., Andrews, K.M., and Reed, R.N., 2006, Efficacy of marking snakes with disposable medical cautery units: Herpetological Review, v. 37, p. 52-54.

Wylie, G.D., Casazza, M.L., and Carpenter, M., 2003, Diet of bullfrogs in relation to predation on giant garter snakes at Colusa National Wildlife Refuge: California Fish and Game, v. 89, p. 139-145.

Wylie, G.D., Casazza, M.L., Gregory, C.J., and Halstead, B.J., 2010, Abundance and sexual size dimorphism of the giant gartersnake (Thamnophis gigas) in the Sacramento Valley of California: Journal of Herpetology, v. 44, p. 94-103.

Wylie, G.D., Casazza, M.L., Halstead, B.J., and Gregory, C.J., 2009, Sex, season, and time of day interact to affect body temperatures of the Giant Gartersnake: Journal of Thermal Biology, v. 34, p. 183-189. 


\section{Glossary}

Apparent survival ( $\phi)$ : The joint probability that an individual survives, and does not permanently leave the study area in a capture-mark-recapture study. Capture-mark-recapture studies, based on marking individuals within a defined study area, estimate apparent survival, not true survival, because it is not possible to know with certainty whether an individual that is not captured for several years died or emigrated offsite. To estimate true survival, additional data, such as the recovery of dead individuals or sighting of emigrants out of the capture-mark-recapture study area, are needed.

Asymptotic size $\left(L_{\infty}\right)$ : The theoretical maximum size an individual approaches as it grows with age in a von Bertalanffy growth model. The asymptotic size describes a population level average, and some individuals may reach maximum sizes greater than the asymptotic size for the population or species.

Bayesian analysis: A method of statistical inference in which Bayes' rule is used to update probability distributions as data are acquired.

Burn-in: The initial samples from a Markov chain, before the chain has converged on a stationary posterior distribution. These samples are discarded from the results of a Bayesian model, because the values of the Markov chain during burn-in is still influenced by the arbitrary starting values.

Capture-mark-recapture: A method of estimating demographic parameters of populations for which individuals cannot be directly counted that involves capturing, marking, and recapturing individuals. Not all capture-mark-recapture methods require physical capture of individuals (for example, camera traps or hair snares for DNA).

Capture probability: The per-survey probability that a specific individual is captured or observed. Centrarchidae: A family of freshwater ray-finned fishes in the order Perciformes that includes the sunfish (for example, largemouth bass, bluegill, pumpkinseed, and crappie).

Coefficient: A parameter of a statistical (regression) model that indicates how the mean response variable changes with the predictor variable; analogous to the slope of a line.

Convergence: The meeting and overlapping of independent Markov chains in a Markov-chain Monte Carlo simulation used to assess whether the stationary posterior distribution has been reached.

Cormack-Jolly-Seber (CJS) model: An open population model that estimates apparent survival by conditioning on the first capture of individuals.

Covariate: A (predictor) variable thought to be correlated with another (response) variable used in a regression analysis.

Credible interval: A specified domain of the posterior probability distribution used for interval estimation. Credible intervals can be interpreted as the probability, confidence, or belief that a parameter or value falls within the specified region.

Cyprinidae: A family of freshwater ray-finned fishes in the order Cypriniformes that includes the carps and true minnows.

Elasticity: The proportional change in population growth rate, $\lambda$, caused by making a very small change in a vital rate parameter (for example, from a survival, growth, or fecundity model) or in the life-stage transition probability in an Integral Projection Model (IPM). Elasticity is generally preferred over sensitivity for evaluating IPMs because it scales the effect of a parameter or transition probability on $\lambda$ by the magnitude of that parameter or transition probability. This makes it possible to compare elasticity among parameters (or among transition probabilities) defined on very different scales. 
Fecundity: The number of offspring produced by a female in a given year, that is, litter size.

Gravid: Pregnant.

Heterogeneity: Differences among units within a group.

Ictaluridae: A family of catfishes in the order Siluriformes that includes the madtoms, bullheads, and channel catfish.

Indicator variable: A variable taking a value of 1 if the corresponding covariate is included in a model or a value of 0 if the corresponding covariate is not included in a model.

Informative prior: A prior probability distribution that expresses prior information or knowledge about a variable, based on data from previous studies or expert knowledge.

Integral Projection Model (IPM): A demographic population model with a discrete time increment and vital rates determined by a continuous state variable. IPMs are often used to model demography using individual size (for example, length, mass) as the state variable.

Jolly-Seber model: An open capture-mark-recapture model that simultaneously estimates survival between sampling periods and the abundance of the population during each sampling period. The Jolly-Seber model attempts to estimate how many individuals were present in the population but not captured during each sampling period.

Litter: The number of young brought forth by an animal with multiple reproductive cycles at one parturition.

Markov-chain Monte Carlo (MCMC): A class of algorithms for sampling from a probability distribution that is based on constructing a Markov chain that has the desired distribution as its equilibrium distribution.

Natricinae: A subfamily of the family Colubridae of snakes, which includes European grass snakes and North American watersnakes and gartersnakes.

Neonate: A newborn.

Parturition: The act or process of giving birth to offspring.

Population growth rate $(\lambda)$ : The change in abundance of a population in year $t+1$ relative to year $t$. $\lambda=1$ represents a stable population (no change in abundance), $\lambda$ less than 1 indicates a declining population, and $\lambda$ greater than 1 indicates an increasing population. $\lambda$ can be calculated from a time series of abundances, for example, $\lambda=N_{\mathrm{t}+1} / N_{t} . \lambda$ for an IPM can also be calculated analytically as the dominant eigenvalue of the IPM kernel, by discretizing the IPM into a large matrix, and using standard methods for calculating the eigenvalues of a matrix.

Population viability analysis (PVA): A model that estimates the probability of a population going extinct, or the size of a population, at some point in the future. Often used to compare the extinction risk for a population or species under alternative management or conservation scenarios.

Posterior distribution: The probability distribution of an unknown quantity conditional on the evidence obtained from an experiment or survey, possibly taking into account relevant information available prior to the experiment or survey.

Posterior probability: The conditional probability assigned to a random event after the relevant evidence or background has been taken into account.

Precinctive: Restricted to a defined geographic area.

Prior probability: The probability distribution that expresses one's uncertainty about an unknown quantity before some evidence (experiment, survey, observation) is taken into account. 
Quadratic: Involving the square of a predictor variable in a regression model.

Recruitment: The process by which offspring born in year $t$ survive and grow to join the censused population in year $t+1$. Only a fraction of the neonates produced in year $t$ will survive to recruit to the censused population in year $\mathrm{t}+1$, and those that survive will have grown to a larger size than their size at birth.

Sensitivity: The change in the population growth rate, $\lambda$, induced by a very small change in a vital rate (for example, the probability a female reproduces) or life-stage transition probability (for example, the probability an 800 millimeter snout-vent-length (SVL) female in year t survives and grows to 850 millimeter SVL in year $\mathrm{t}+1$ ). Sensitivities are used to compare the relative importance of different vital rates or life-stage transition probabilities for population growth. See also Elasticity.

Snout-vent length (SVL): A measure of body length commonly used in reptiles. Length is measured from the anterior tip of the snout to the vent or opening of the cloaca. SVL is generally preferred over total body length because in reptiles many individuals lose portions of their tail during their life (likely from attempted predation); thus SVL is comparable among individuals. Survival: The rate or probability at which individuals alive at time $t$ remain alive until time $t+1$. Temporary emigration: The temporary movement of individuals outside the sampled area. Transition probability: In an IPM, the likelihood that a distribution of individual sizes, $x$, transitions from to a new distribution of sizes, $x^{\prime}$, at time $\mathrm{t}+1$. This includes the survival and growth of individuals that are already present in the population and the production of new individuals by adult females.

Uninformative prior: A prior probability distribution that expresses vague or general information about a variable, typically with the goal of assigning equal probabilities to all possibilities. Vital rate: Also called a demographic rate; the rates of survival, growth, and reproduction in a population. Vital rates are often linked to individual states or characteristics, such as size, age, or sex, to allow researchers to simulate the way in which the population will change over time. von Bertalanffy Growth Model: A model of growth in body size where individuals initially increase rapidly in size. Then growth slows as individuals approach an asymptotic size. This model is commonly used to describe the growth of fish, reptiles, and other ectotherms. 
Publishing support provided by the U.S. Geological Survey

Science Publishing Network, Tacoma Publishing Service Center

For more information concerning the research in this report, contact the Director, Western Ecological Research Center

U.S. Geological Survey

3020 State University Drive East

Sacramento, California 95819

https://www.werc.usgs.gov/ 
응.

항

3

$\frac{\infty}{1}$ 